\title{
Performance Analysis of Statistical Approaches and NMF Approaches for Speech Enhancement
}

\author{
Ravi Kumar Kandagatla, Sr.Assistant Professor \\ Department of ECE, Lakireddy Bali Reddy College of Engineering, Mylavaram, India \\ Email: 2k6ravi@gmail.com \\ P V Subbaiah \\ Professor of ECE, V.R. Siddhartha Engineering College, Vijayawada, India \\ Email: pvsubbaiah@vrsiddhartha.ac.in
}

Received: 03 May 2019; Accepted: 22 May 2019; Published: 08 July 2019

\begin{abstract}
Super-Gaussian Based Bayesian Estimators plays significant role in noise reduction. However, the traditional Bayesian Estimators process only DFT spectral amplitude of noisy speech and the phase is left unprocessed. While deriving Bayesian estimators, consideration of phase information provides improved results. The main objective of this paper is twofold. Firstly, the Super-Gaussian based Complex speech coefficients given Uncertain Phase (CUP) based Bayesian estimators are compared under different noise conditions like White noise, Babble noise, Pink noise, Modulated Pink noise, Factory noise, Car noise, Street noise, F16 noise and M109 noise. Secondly, a novel speech enhancement method is proposed by combining CUP estimators with different NMF approaches and online bases updation. The statistical estimators show less effective results under completely non-stationary assumptions. Non-negative Matrix Factorization (NMF) based algorithms show better performance for non stationary noises. The drawback of NMF is, it requires training and/or requires clean speech and noise signals. This drawback can be overcome by taking the advantages of both statistical approaches and NMF approaches. Such approaches like Posteriori Regularized NMF (PR-NMF), Weibull Rayleigh NMF (WR-NMF), Nakagami Rayleigh (NR-NMF), CUP estimator with Gamma and Generalized Gamma distributions + NMF + Online bases Update (CUP-GG + NMF + OU) and CUP-GG + WR-NMF / $\mathrm{NR}-\mathrm{NMF}+\mathrm{OU}$ are considered for comparison. The objective of this paper is to analyze the performance of speech enhancement methods using Bayesian estimators, NMF approaches, Combination of statistical and NMF approaches. The objective performance measures Perceptual Evaluation of Speech Quality (PESQ), ShortTime Objective Intelligibility (STOI), Signal to Noise Ratio (SNR), Signal to Distortion Ratio (SDR), Segmental SNR (Seg SNR) are considered for comparison.
\end{abstract}

Index Terms - Non-Negative Matrix Factorization, CUP Estimator, Noise Reduction, PESQ

\section{INTRODUCTION}

Speech is an important means for communicating thoughts between person to person / human to machine. But the speech communicated, is affected/corrupted by the noise present in the environment. Speech enhancement is the technique used in reducing noise from corrupted signal. The objective of speech enhancement is twofold in which it can improve quality as well as intelligibility. Quality deals with SNR of the signal and intelligibility deals with how much percent of words will be understood correctly.

Traditional speech enhancement approaches include Minimum Mean Square Error (MMSE) estimators and Bayesian estimators assume the speech Discrete Fourier Transform (DFT) spectral coefficients and noise spectral coefficients as Gaussian distribution [1-2]. Later it is observed that, improved results are obtained by considering Super-Gaussian (Gamma, Rayleigh, Chi, Exponential, Nakagami) distributions rather than assuming Gaussian for speech and noise spectral coefficients [3-,4]. Thus in this work, the comparison of statistical estimators namely Harmonic Regeneration for Noise Reduction (HRNR), Harmonic Regeneration for Noise Reduction with Speech Presence Uncertainty Estimator (HRNR-SPU) is discussed [5]. Traditional Bayesian estimators process only DFT amplitudes and phase is left unprocessed. In enhanced speech signal reconstruction, the unprocessed phase of noisy speech is used [6]. Later in [7] the Bayesian estimators that use phase information for amplitude estimation is derived. It is observed that performance of Bayesian estimators is improved by considering phase information [7-8]. In this work, Super Gaussian based estimators which used phase information like CUP, CUP-NG and CUP-GG are considered for comparison.

It is observed that template based approaches like NMF approach perform better under non-stationary noises. But the disadvantage is that it requires apriori information of speech and noise which is not required in statistical approaches. By combining the advantages of 
both statistical and NMF approaches, the speech enhancement methods like PR-NMF, WR-NMF and GR NMF are considered for comparison.

In combined approach, the traditional algorithm for speech enhancement [7] is used in first stage. In the second stage, different NMF approaches are considered and combined with online bases update. The performance of proposed method is compared with traditional and recent approaches of speech enhancement.

The paper is organized as follows. In Section II different statistical approaches are discussed. In Section III the variation of NMF approaches are discussed. In Section IV the speech enhancement approach using combination of statistical and NMF approaches with online bases update is discussed. Section V discusses the Results and Section V1 provides conclusion on the paper.

\section{StATISTICAL APPROACHES FOR SPEECH ENHANCEMENT}

The most traditional statistical approach for speech enhancement is Wiener Filter approach. The gain of the wiener filter is obtained by assuming speech DFT coefficients as Gaussian and under MMSE sense. The gain depends on priori SNR. But the disadvantage is that the calculation of priori SNR of current frame depends on past frame and thus the gain applied to current frame depends on past frame. To overcome this problem, new speech enhancement approaches TSNR and HRNR are proposed in literature. The HRNR approach shows inferior performance under low SNR conditions. Thus in this paper, HRNR method is combined with Speech Presence Uncertainty (SPU) estimator. It is observed that there is performance improvement in HRNR-SPU approach.

The above mentioned approaches process only noisy amplitudes but phase is left unprocessed and the unprocessed noisy phase is considered for reconstruction. It is noted that the above mentioned algorithms work under Gaussian assumption. Later, it is observed that using Super-Gaussian assumption based estimators provide better results as the distribution gives best fit to the data. In this work, Super-Gaussian based estimators are derived by considering phase information and different CUP estimators are taken for comparison [5-8].

The Complex speech coefficients given Uncertain Phase (CUP) estimator is derived by assuming speech as Chi distribution and noise as Gaussian. CUP estimator estimates the amplitude by considering phase information. The phase information of clean speech is estimated by noisy signal phase. Where as in CUP-NG and CUP-GG, it is assumed that noise spectral coefficients follow Generalized Gamma distribution where as speech spectral coefficients follow Nakagami and Gamma respectively [9]. The equations of gain are summarized in table I.

Let $s(n)$ be the clean speech, $n(n)$ be the noise and $y(n)$ be the noisy speech. Assume the additive noise and the noisy speech in STFT domain be $Y(k, i)$, the clean speech $S(k, i)$ and noise be $N(k, i)$, then noisy speech can be mathematically represented as

$$
Y(k, i)=S(k, i)+N(k, i)
$$

Where $k, i$ are the indices of frequency and time respectively. The complex spectral coefficients of clean speech, noisy speech and noise are represented as

$$
\begin{aligned}
& Y=\operatorname{Re}^{j \phi_{Y}} \\
& S=A e^{j \phi_{S}} \\
& W=N e^{j \phi_{N}}
\end{aligned}
$$

Where $Y, S, W$ represents the complex spectral coefficients and $\mathrm{R}, \mathrm{A}, \mathrm{N}$ be the amplitudes and $\mathrm{e}^{j \phi_{Y}}, e^{j \phi_{S}}$, $e^{j \phi_{N}}$ be the phases of noisy speech, clean speech and noise respectively. The priori knowledge of clean speech is estimated from noisy speech using [8]. The estimate of clean speech is obtained using $E\left(\left.S\right|_{Y, \tilde{\varphi}_{S}}\right)$, where $\tilde{\varphi}_{S}$ denotes the priori knowledge of the clean speech estimate. Also from [2], it is noted that by taking compressed amplitudes with parameter $\beta$ provides perceptual benefit in enhancement process. Thus including compressed amplitude and the final estimate of the enhanced speech is obtained by calculating

$$
\begin{aligned}
\hat{S}^{(\beta)}= & E\left(A^{\beta} e^{j \Phi_{S}} \mid y, \tilde{\phi}_{S}\right)= \\
& \int_{0}^{\infty} \int_{0}^{2 \pi} a^{\beta} e^{j \Phi_{S}} p_{A, \Phi_{S} \mid y, \tilde{\phi}_{S}}\left(a, \phi_{S} \mid y, \tilde{\phi}_{S}\right) d \phi_{S} d a
\end{aligned}
$$

By using Bayes rule, the posterior function can be modeled using

$$
\begin{gathered}
p_{A, \Phi_{S} \mid y, \tilde{\phi}_{S}}\left(a, \phi_{S} \mid y, \tilde{\phi}_{S}\right)=\frac{p_{Y, A, \Phi_{S}, \tilde{\Phi}_{S}}\left(y, a, \phi_{S}, \tilde{\phi}_{S}\right)}{p_{Y, \Phi_{S}}\left(y, \tilde{\phi}_{S}\right)} \\
=\frac{p_{Y \mid S, \tilde{\phi}_{S}}\left(y \mid a, \phi_{S}, \tilde{\phi}_{S}\right) p_{A, \Phi_{S}, \tilde{\Phi}_{S}}\left(a, \phi_{S}, \tilde{\phi}_{S}\right)}{\iint p_{Y \mid s, \tilde{\phi}_{S}}\left(y \mid a, \phi_{S}, \tilde{\phi}_{S}\right) p_{A, \Phi_{S}, \tilde{\Phi}_{S}}\left(a, \phi_{S}, \tilde{\phi}_{S}\right) \operatorname{dad} \phi_{S}}
\end{gathered}
$$

Assuming that amplitudes and phases are mutually dependent, then the PDF of amplitudes can be written as

$$
\begin{array}{r}
p_{A, \Phi_{S}, \tilde{\Phi}_{S}}\left(a, \phi_{S}, \tilde{\phi}_{S}\right)=p_{A}(a) p_{\Phi_{S}, \tilde{\Phi}_{S}}\left(\phi_{S}, \tilde{\phi}_{S}\right) \\
=p_{A}(a) p_{\Phi_{S} \mid \tilde{\phi}_{S}}\left(\phi_{S} \mid \tilde{\phi}_{S}\right) p_{\Phi_{S}}\left(\tilde{\phi}_{S}\right)
\end{array}
$$


By substituting eq.(5) in eq.(4), the posterior results in

$$
\begin{aligned}
& p_{A, \Phi_{S} \mid y, \tilde{\phi}_{S}}\left(a, \phi_{S} \mid y, \tilde{\phi}_{S}\right) \\
& =\frac{p_{Y / s}\left(y \mid a, \phi_{S}\right) p_{A}(a) p_{\Phi_{S}, \tilde{\varphi}_{S}}\left(\phi_{S}, \tilde{\phi}_{S}\right)}{\iint p_{Y \mid s}\left(y \mid a, \phi_{S}\right) p_{A}(a) p_{\Phi_{S}, \tilde{\phi}_{S}}\left(\phi_{S}, \tilde{\phi}_{S}\right) d a d \phi_{S}}
\end{aligned}
$$

Now assume the speech spectral coefficients PDF $p_{A}(a)$ as Gamma, Nakagami and noise spectral coefficients, $p_{Y \mid S}(y \mid a)$, as Gaussian, Generalized Gamma distribution. The resultant estimators gain [see Appendix A, Appendix B for proofs and for information regarding different parameters of gain functions] is obtained as given in Table I.

\begin{tabular}{|c|c|}
\hline Estimator & Gain Equation \\
\hline CUP [8] & $\hat{S}^{(\beta)}=\left(\sqrt{\frac{1}{2} \frac{\xi}{\mu+\xi} \sigma_{V}^{2}}\right)^{\beta} \frac{\Gamma(2 \mu+\beta)}{\Gamma(2 \mu)} \times \frac{\int_{0}^{2 \pi} e^{j \phi^{S}} e^{\frac{v^{2}}{4}} D_{(-2 \mu-\beta)}(v) p\left(\phi^{S} \mid \tilde{\phi}^{S}\right) d \phi^{S}}{\int_{0}^{2 \pi} e^{\frac{v^{2}}{4}} D_{(-2 \mu)}(v) p\left(\phi^{S} \mid \tilde{\phi}^{S}\right) d \phi^{S}}$ \\
\hline CUP-NG[9] & $\begin{array}{l}\hat{S}^{(\beta)}=E\left(A^{\beta} e^{j \Phi_{S}} \mid y, \tilde{\phi}_{S}\right)= \\
=\frac{\Gamma(2 k+2 v+\beta-1)}{\Gamma(2 k+2 v-1)}\left(2\left(\frac{k}{\xi \sigma_{N}^{2}}+\frac{\xi \mu}{\sigma_{S}^{2}}\right)\right)^{-\left(\frac{\beta}{2}\right)} \frac{\int_{0}^{2 \pi} e^{j \phi_{S}} \exp \left(\frac{r^{2}}{\sigma_{N}^{2}}\right) \exp \left(\frac{v^{2}}{2}\right) D_{-(2 v+2 k+\beta-1)}(v) p_{\Phi_{S} \mid \tilde{\phi}_{S}}\left(\phi_{S} \mid \tilde{\phi}_{S}\right) d \phi_{S}}{\int_{0}^{2 \pi} \exp \left(\frac{r^{2}}{\sigma_{N}^{2}}\right) \exp \left(\frac{v^{2}}{2}\right) D_{-(2 v+2 k-1)}(v) p_{\Phi_{S} \mid \tilde{\phi}_{S}}\left(\phi_{S} \mid \tilde{\phi}_{S}\right) d \phi_{S}}\end{array}$ \\
\hline CUP-GG[9] & $\begin{array}{l}\hat{S}^{(\beta)}=E\left(A^{\beta} e^{j \Phi_{S}} \mid y, \tilde{\phi}_{S}\right)= \\
\quad=\frac{\Gamma(2 v+k+\beta-1)}{\Gamma(2 v+k-1)}\left(\frac{2 \mu}{\sigma_{N}^{2}}\right)^{-\left(\frac{\beta}{2}\right)} \frac{\int_{0}^{2 \pi} e^{j \phi_{S}} \exp \left(\frac{v^{2}}{2}\right) D_{-(2 v+k+\beta-1)}(v) p_{\Phi_{S} \mid \tilde{\phi}_{S}}\left(\phi_{S} \mid \tilde{\phi}_{S}\right) d \phi_{S}}{\int_{0}^{2 \pi} \exp \left(\frac{v^{2}}{2}\right) D_{-(2 v+k-1)}(v) p_{\Phi_{S} \mid \tilde{\phi}_{S}}\left(\phi_{S} \mid \tilde{\phi}_{S}\right) d \phi_{S}}\end{array}$ \\
\hline
\end{tabular}

Table 1. Gain functions of different cup Estimators

\section{NMF APPROACHES FOR SPEECH ENHANCEMENT}

NMF based speech enhancement methods deal well with non-stationary noises. In NMF techniques the priori information is given by assuming statistical distributions of speech and noise. The distributions are selected such that, they provide better fit and less Kullback-Leibler (KL) divergence. The assumption of Gaussian noise suits well as sum of different noises tends to Gaussian (Central Limit Theorem). If DFT coefficients follow Gaussian, then the magnitudes follow Rayleigh distribution. In $\mathrm{NMF}$, the factorization is performed by considering magnitudes. Thus the penalties or priori for noise is assumed as Rayleigh distribution. Similarly speech spectral magnitudes better fit under super Gaussian assumption and here Nakagami and Weibull distributions are assumed. NMF update rules are obtained by gradient descent algorithm which minimizes Kullback-Leibler divergence. The complete algorithm of posterior regularized NMF is given below.

Basically in NMF analysis, the STFT magnitudes of noisy speech, i.e., a spectrogram matrix $X$ of $M$ rows and $N$ columns is factorized into product of two matrices (Note that $M, N$ also indicates the no. of frequency bins and frames respectively). The two matrices contain nonnegative elements and let the two matrices be $W$ (Bases Matrix) and $H$ (Encoding Matrix). Here $W$ is a matrix of size $M$ rows and $\mathrm{r}$ columns and $H$ is a matrix of size $\mathrm{r}$ rows and $N$ columns, where $\mathrm{r}$ is the sum of number of speech $\left(r_{s}\right)$ and noise $\left(r_{n}\right)$ bases. Each of $W, H$ individually contains speech and noise components. They are represented mathematically as $W=\left[W_{S} W_{n}\right]$, $H=\left[H_{S}^{T} H_{N}^{T}\right]^{T}$, where size of $W, H$ matrices is $M \times\left(r_{s}+r_{n}\right)$, where suffixes indicate speech and noise.

Let $s(n)$ the clean speech, $n(n)$ be the noise and $x(n)$ be the noisy speech. For better fit, the noise PDF coefficients are modeled by Rayleigh prior distribution. The Rayleigh PDF is given as

$$
f(x ; \sigma)=\frac{x}{\sigma^{2}} e^{\frac{-x^{2}}{2 \sigma^{2}}}, x \geq 0
$$

Here the parameter $\sigma$ is estimated by using Maximum likelihood parameter estimation and it would be given as 


$$
\sigma^{2}=\frac{1}{2 N} \sum_{i=1}^{N} x_{i}^{2}
$$

Apply negative logarithm on both sides

$$
\begin{aligned}
-\log (f(x ; \sigma)) & =-\log \left(\frac{x}{\sigma^{2}} e^{\frac{-x^{2}}{2 \sigma^{2}}}\right) \\
& =-\log \left(\frac{x}{\sigma^{2}}\right)+\frac{x^{2}}{2 \sigma^{2}}
\end{aligned}
$$

Thus, the penalty/regularization considered with the noise magnitudes prior distribution is

$$
\begin{aligned}
\Lambda_{N}=\Lambda_{S_{1}}=-\log (f(x ; \sigma)) & \\
& =\frac{x^{2}}{2 \sigma^{2}}-\log \left(\frac{x}{\sigma^{2}}\right)
\end{aligned}
$$

The magnitudes of the speech signal are better fit by assuming powerful Weibull distribution from [3]. It can be shown that the smoothed KL divergence error is less for Weibull than the other distributions. Thus magnitudes of speech signal is modeled using Weibull distribution with adjustable scale parameter as

$$
f(x \mid \chi, \delta)=\frac{\delta}{\chi}\left(\frac{x}{\chi}\right)^{\delta-1} \exp \left(-\left(\frac{x}{\chi}\right)^{\delta}\right), x \geq 0
$$

With the estimated scale parameter as

$$
\chi=\sqrt{\frac{\lambda_{s}}{\Gamma(1+2 / \delta)}}
$$

Where $\lambda_{s}(k)=E\left[\left|X_{k}\right|^{2}\right]$, (here $k$ is frequency bin which is omitted in formula). The regularization / penalty term for the speech sample is obtained by taking the negative logarithm on both sides.

$$
\begin{aligned}
& \Lambda_{S P}=\Lambda_{S_{2}}=-\log (f(x \mid \chi, \delta)) \\
&=\left(\frac{x}{\chi}\right)^{\delta}-\log \left(\frac{\delta x^{\delta-1}}{\chi^{\delta}}\right), x \geq 0
\end{aligned}
$$

The proposed algorithm is implemented with the following step by step process. Here the Weibull distribution is used and can replace the same with Nakagami to implement Nakagami Rayleigh NMF (NRNMF).

\section{Speech Enhancement Algorithm using WR-NMF (Weibull Rayleigh NMF)}

$\mathrm{X} \in \mathbb{R}_{+}^{\mathrm{f} \times \mathrm{t}}, \%$ Observed normalized data

$$
\begin{gathered}
\Lambda_{S} \in \mathbb{R}_{+}^{f \times t}, s \in\left\{1, \ldots . N_{S}\right\} \% \Lambda_{S} \text { - penalties, } \\
N_{S}-\text { Number of sources } \\
\Lambda_{S(\text { New })}=0 \\
\Lambda_{\mathrm{S}_{1}}=\Lambda_{\mathrm{N}(\text { old })}=\frac{\mathrm{X}^{2}}{2 \sigma^{2}}-\log \left(\frac{\mathrm{X}}{\sigma^{2}}\right) \\
\Lambda_{S_{2}}=\Lambda_{S P(\text { old })}=\left(\frac{\hat{\mathrm{X}}_{\mathrm{S}_{2}}}{\chi}\right)^{\delta}-\log \left(\frac{\delta\left(\hat{\mathrm{X}}_{\mathrm{S}_{2}}\right)^{\delta-1}}{\chi^{\delta}}\right)
\end{gathered}
$$

where, $\delta=0.68$

\section{Repeat}

For all s do

$\tilde{\Lambda}_{\text {S(old })} \leftarrow \exp \left\{-\Lambda_{s}\right\}, \%$ Update penalties using LMS (22)

$$
\begin{gathered}
\tilde{\Lambda}_{S}=(1-\mu) \Lambda_{s(\text { old })}+\mu \Lambda_{s(\text { New })} \\
\Lambda_{s(\text { old })}=\Lambda_{s(\text { New })} \\
\mathrm{X}_{\mathrm{s}} \leftarrow \mathrm{X} \tilde{\Lambda}_{\mathrm{s}}
\end{gathered}
$$

\section{End For}

For all s do

$$
\Gamma \leftarrow \sum_{\mathrm{s}}\left(\mathrm{W}_{(\mathrm{s})} \mathrm{H}_{(\mathrm{s})}\right) \tilde{\Lambda}_{\mathrm{s}}
$$

$$
\mathrm{z} \leftarrow \frac{\mathrm{X}_{\mathrm{s}}}{\Gamma}
$$

$$
\mathrm{W}_{(\mathrm{s})} \leftarrow \mathrm{W}_{(\mathrm{s})} \frac{\mathrm{Z}_{\mathrm{s}} \mathrm{H}_{(\mathrm{s})}^{\mathrm{T}}}{1 \mathrm{H}_{(\mathrm{s})}^{\mathrm{T}}}
$$

$$
\mathrm{H}_{(\mathrm{s})} \leftarrow \mathrm{H}_{(\mathrm{s})}\left(\mathrm{W}_{(\mathrm{s})}^{\mathrm{T}} \mathrm{Z}_{\mathrm{s}}\right)
$$

\section{End For}

\section{Reconstruction}

\section{For all s do}

$$
M_{s} \leftarrow \frac{W_{(s)} H_{(s)}}{W H}, \quad \% \text { Computes the Filter }
$$




$$
\hat{X}_{s} \leftarrow M_{s} X, \quad \% \text { Filter Mixture }
$$

$x_{s} \leftarrow \operatorname{ISTFT}\left(\hat{X}_{s}, \angle X, P\right) \quad \% \mathrm{P}-\mathrm{STFT}$ parameters

Update $\chi$

$$
\chi=\sqrt{\frac{\lambda_{s}}{\Gamma(1+2 / \delta)}}
$$

Where, $\lambda_{s}(k)=E\left[\left|X_{k}\right|^{2}\right]$

\section{End update}

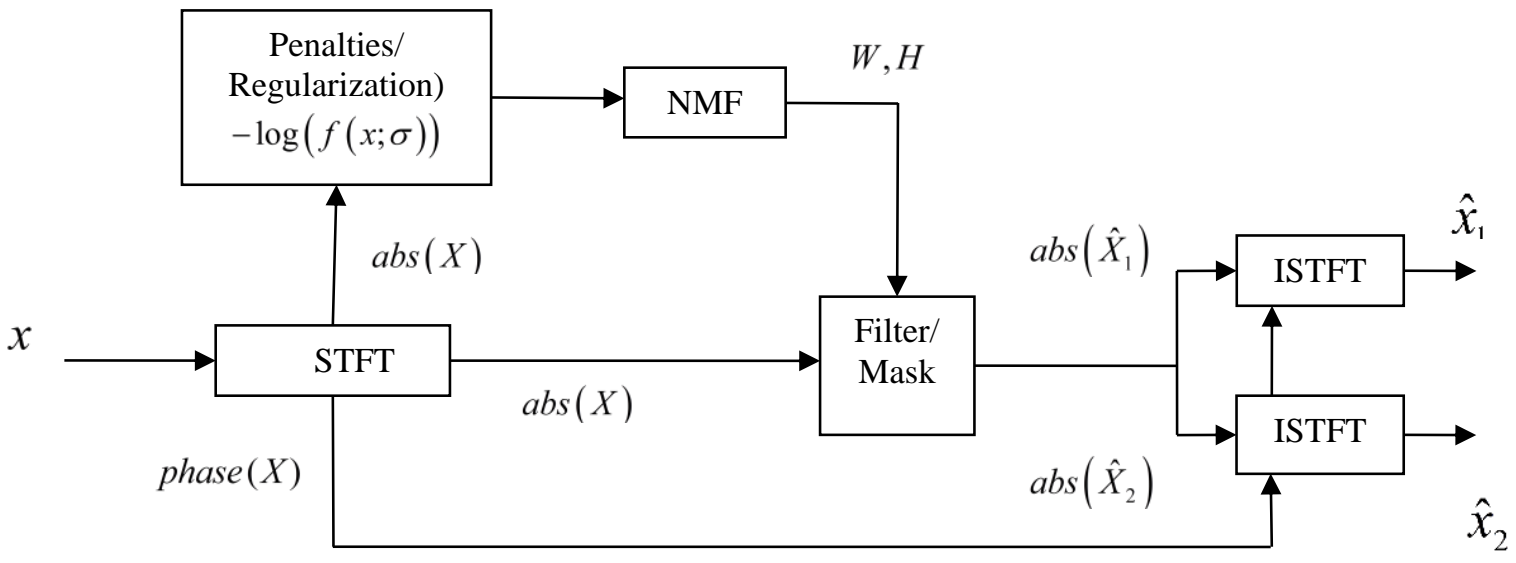

Fig.1. Block Diagram for Posteriori Regularization Based Speech Enhancement

Table 2. Different distributions and its negative logarithms in mathematical form

\begin{tabular}{ccc}
\hline PDF & Regularization & Parameters \\
$f(x ; \sigma)$ & $-\log (f(x ; \sigma))$ & \\
& & \\
\end{tabular}

$$
\Lambda_{S_{1}}=\Lambda_{N(\text { old })}=\frac{\hat{\mathrm{X}}_{\mathrm{S}_{1}}^{2}}{2 \sigma^{2}}-\log \left(\frac{\hat{\mathrm{X}}_{\mathrm{S}_{1}}}{\sigma^{2}}\right)
$$

$$
\begin{gathered}
\Lambda_{S_{2}}=\Lambda_{S P(\text { old })}=\left(\frac{\hat{X}_{S_{2}}}{\chi}\right)^{\delta}-\log \left(\frac{\delta\left(\hat{X}_{S_{2}}\right)^{\delta-1}}{\chi^{\delta}}\right) \\
\Lambda_{s(\text { New })}=\exp \left(-\Lambda_{s(\text { old })}\right)
\end{gathered}
$$

End For

\section{Untill Convergence}

Return: Time domain signals $x_{s}$
The above mentioned algorithm is completely described with the help of Block diagram as shown in Fig.1

$$
\begin{aligned}
& \text { Nakagami } \\
& \frac{2}{\Gamma(\mu)}\left(\frac{\mu}{\sigma^{2}}\right)^{\mu} x^{2 \mu-1} \exp \left(-\frac{\mu}{\sigma^{2}} x^{2}\right) \quad-\log \left(\frac{2}{\Gamma(\mu)}\right)-\mu \log \left(\frac{\mu}{\sigma^{2}}\right)-(2 \mu-1) \log x+\left(\frac{x^{2} \mu}{\sigma^{2}}\right) \quad \sigma^{2}=\frac{1}{n} \sum_{i=1}^{n} x_{i}^{2} \\
& \mu<1 \text { allows Super- Gaussian } \\
& \frac{\delta}{\chi}\left(\frac{x}{\chi}\right)^{\delta-1} \exp \left(-\left(\frac{x}{\chi}\right)^{\delta}\right) \\
& \begin{array}{l}
\text { Rayleigh } \\
\frac{x}{\sigma^{2}} e^{\frac{-x^{2}}{2 \sigma^{2}}}
\end{array} \\
& \left(\frac{x}{\chi}\right)^{\delta}-\log \left(\frac{\delta x^{\delta-1}}{\chi^{\delta}}\right) \\
& \delta=0.68 \\
& \begin{array}{l}
\chi=\sqrt{\frac{\lambda_{s}}{\Gamma(1+2 / \delta)}} \\
\lambda_{s}(k)=E\left[\left|X_{k}\right|^{2}\right]
\end{array} \\
& \sigma^{2}=\frac{1}{2 N} \sum_{i=1}^{N} x_{i}^{2}
\end{aligned}
$$


In the first step of Fig, 1, Short Time Fourier Transform (STFT) is applied and the distributions of magnitude spectral coefficients are assumed. Negative logarithms of assumed distributions are considered for providing regularization with NMF approach

\section{COMBination OF Novel Estimator AND NMF APPROACH FOR SPEECH ENHANCEMENT}

The proposed speech enhancement process is performed in two steps. In the first step, the speech enhancement method proposed in [12] is used to enhance the noisy speech. In the later stage, the NMF with online bases update is used for further processing. The added feature in this method is estimating the gain using novel phase based CUP estimator. The complete process of proposed method is shown in Fig.2. The estimated speech $\hat{S}$ and $\hat{N}$ is obtained using

$$
\begin{aligned}
& \hat{S}=\tilde{W}_{s} \tilde{H}_{s} \\
& \tilde{N}=\tilde{W}_{n} \tilde{H}_{n}
\end{aligned}
$$

Where the basis matrix and weight matrix updates are obtained with KL divergence as follows

$$
\begin{gathered}
\mathrm{W}_{(\mathrm{s})} \leftarrow \mathrm{W}_{(\mathrm{s})} \frac{\mathrm{Z}_{\mathrm{s}} \mathrm{H}_{(\mathrm{s})}^{\mathrm{T}}}{1 \mathrm{H}_{(\mathrm{s})}^{\mathrm{T}}} \\
\mathrm{H}_{(\mathrm{s})} \leftarrow \mathrm{H}_{(\mathrm{s})}\left(\mathrm{W}_{(\mathrm{s})}^{\mathrm{T}} \mathrm{Z}_{\mathrm{s}}\right)
\end{gathered}
$$

In $\beta$-NMF, the update is done as follows

$$
\begin{gathered}
H \leftarrow H \cdot \frac{W^{T}\left[(W H)^{\cdot(\beta-2)} \cdot V\right]}{W^{T}[W H]^{(\beta-1)}} \\
W \leftarrow W \cdot \frac{\left[(W H)^{(\beta-2)} \cdot V\right] H^{T}}{[W H]^{(\beta-1)} H^{T}}
\end{gathered}
$$

HRNR - The Harmonic Regeneration for Noise Reduction, is one in which the gain depends on priori SNR. The priori SNR is calculated such that the current frame priori SNR depends on current samples. A non linear function is used for generating harmonics artificially.

HRNR-SPU- The HRNR gain is modified according to Speech Presence Uncertainty estimator Gain.

$P R-N M F$ - It is the Posterior Regularized NMF technique. In this the importance of regularization in NMF approach is discussed in [11].

$W R-N M F$ - It is similar to PR-NMF approach, but regularization is provided by assuming magnitudes of speech and noise as Weibull and Rayleigh distributions respectively and negative logarithm of assumed distributions is used for providing regularization.

CUP Estimator- It is derived under statistical assumption that speech spectral coefficients follow Chi distribution and noise spectral coefficients follow Gaussian distribution.

CUP- NG Estimator- It is derived under statistical assumption that speech spectral coefficients follow Nakagami distribution and noise spectral coefficients follow Generalized Gamma distribution.

CUP-GG Estimator- It is derived under statistical assumption that speech spectral coefficients follow Gamma distribution and noise spectral coefficients follow Generalized Gamma distribution

$S E+N M F+O U-$ It is the cascade of Speech Enhancement (A highly Non-Stationary Speech Enhancement) and NMF approach with online bases update.

$S E+N M F+C U P-G G+O U-C U P$ estimator is used in the second stage of $\mathrm{SE}+\mathrm{NMF}+\mathrm{OU}$

$S E+\beta-N M F+C U P-G G+O U-$ It is same as $\mathrm{SE}+\mathrm{NMF}+$ CUP-GG+OU and the NMF variant $\beta$-NMF is used.

$S E+W R-N M F+C U P-G G+O U-$ In the approach $S E+N M F+C U P-G G+O U$ the NMF is modified as WR$\mathrm{NMF}$

$\mathrm{SE}+\mathrm{NR}-\mathrm{NMF}+\mathrm{CUP}-\mathrm{GG}+\mathrm{OU}-\mathrm{In}$ the approach $S E+N M F+C U P-G G+O U$ the NMF is modified as NRNMF. 


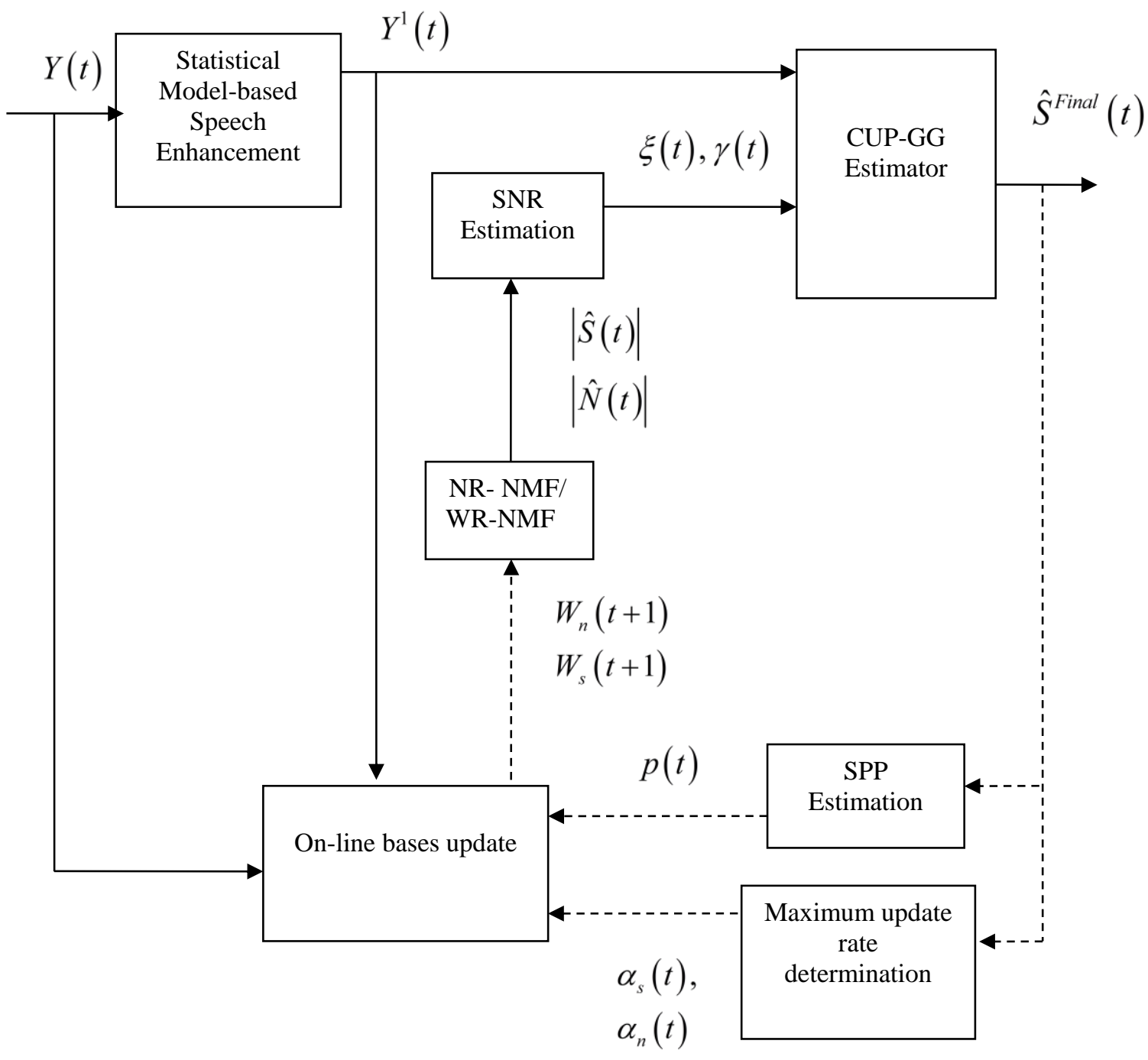

Fig.2. Block Diagram for Proposed Method

\section{RESUlt ANALYSIS}

The performance evaluation of the proposed methods Bayesian estimators and different NMF approaches is implemented by taking the speech samples from NOIZEUS speech corpus data base. Out of 30 sentences considered, 15 sentences were spoken by male speakers and 15 sentences were spoken by female speakers and the signals are corrupted additively under different noise environments. In analysis and synthesis of signal, Hanning window with $75 \%$ overlap is considered. In this work, white, pink and babble noise are taken from Noisex Database and corrupted with the clean speech signals of NOIZEUS database at different input SNRs of $-10 \mathrm{~dB},-5$ $\mathrm{dB}, 0 \mathrm{~dB}, 5 \mathrm{~dB}, 10 \mathrm{~dB}, 15 \mathrm{~dB}$. The objective performance measures Perceptual Evaluation of Speech Quality (PESQ) and Short Time Objective Intelligibility (STOI) is considered for comparison of different speech enhancement methods. For better evaluation, $\triangle \mathrm{PESQ}$ and \% Improvement in STOI are considered for comparison. $\triangle$ PESQ is obtained by taking the difference between the PESQ measured between enhanced speech, clean speech and clean speech, noisy speech. \% STOI is measured as the percentage of improvement in STOI value when STOI value of clean speech, noisy speech is compared with STOI value of clean speech, enhanced speech.

Performance evaluation of speech enhanced methods HRNR, HRNR-SPU, CUP, CUP-GG, CUP-NG, $\mathrm{NMF}+\mathrm{OU}, \mathrm{SE}+\mathrm{CUP}+\mathrm{WR} / \mathrm{NRNMF}+\mathrm{OU}$, is discussed and performance comparison is given in Table 3 to Table 29. 
Table 3. comparison of Average PESQ, $\triangle$ PESQ performance measures for the 30 speech signals corrupted by white noise under Input SNRs of -10 $\mathrm{dB},-5 \mathrm{~dB}, 0 \mathrm{~dB}, 5 \mathrm{~dB}, 10 \mathrm{~dB}, 15 \mathrm{~dB}$

\begin{tabular}{|c|c|c|c|c|c|c|c|}
\hline Input SNR in dB & $\begin{array}{c}\text { Performance } \\
\text { Measure }\end{array}$ & -10 & -5 & $\mathbf{0}$ & 5 & 10 & 15 \\
\hline Without Processing & PESQ & 1.35 & 1.45 & 1.33 & 1.92 & 2.08 & 2.27 \\
\hline \multirow{2}{*}{$\mathrm{SE}+\mathrm{CUP}-\mathrm{GG}+\mathrm{NR}-\mathrm{NMF}+\mathrm{OU}$} & PESQ & 1.64 & 2.01 & 2.51 & 3.17 & 3.27 & 3.41 \\
\hline & $\Delta$ PESQ & 0.29 & 0.56 & 1.18 & 1.25 & 1.19 & 1.14 \\
\hline \multirow{2}{*}{$\mathrm{SE}+\mathrm{CUP}-\mathrm{GG}+\mathrm{WR}-\mathrm{NMF}+\mathrm{OU}$} & PESQ & 1.62 & 1.96 & 2.55 & 3.14 & 3.25 & 3.39 \\
\hline & $\triangle$ PESQ & 0.27 & 0.51 & 1.19 & 1.22 & 1.17 & 1.12 \\
\hline \multirow{2}{*}{$\mathrm{SE}+\mathrm{CUP}-\mathrm{GG}+\boldsymbol{\beta}-\mathrm{NMF}+\mathrm{OU}$} & PESQ & 1.6 & 1.93 & 2.52 & 3.13 & 3.23 & 3.38 \\
\hline & $\triangle$ PESQ & 0.25 & 0.48 & 1.19 & 1.21 & 1.15 & 1.11 \\
\hline \multirow{2}{*}{ SE+CUP GG+NMF[9] } & PESQ & 1.58 & 1.94 & 2.49 & 3.11 & 3.19 & 3.39 \\
\hline & $\triangle$ PESQ & 0.23 & 0.49 & 1.16 & 1.19 & 1.11 & 1.12 \\
\hline \multirow{2}{*}{$\mathrm{NMF}+\mathrm{OU}[12]$} & PESQ & 1.52 & 1.86 & 2.11 & 2.69 & 2.83 & 2.99 \\
\hline & $\Delta$ PESQ & 0.17 & 0.41 & 0.78 & 0.77 & 0.75 & 0.72 \\
\hline \multirow{2}{*}{ CUP-GG[9] } & PESQ & 1.54 & 1.91 & 2.25 & 2.9 & 2.99 & 3.16 \\
\hline & $\Delta$ PESQ & 0.19 & 0.46 & 0.92 & 0.98 & 0.91 & 0.89 \\
\hline \multirow{2}{*}{ CUP-NG[9] } & PESQ & 1.52 & 1.88 & 2.17 & 2.8 & 2.9 & 3.03 \\
\hline & $\Delta$ PESQ & 0.17 & 0.43 & 0.84 & 0.88 & 0.82 & 0.76 \\
\hline \multirow{2}{*}{ CUP[8] } & PESQ & 1.49 & 1.84 & 2.06 & 2.67 & 2.79 & 2.96 \\
\hline & $\triangle$ PESQ & 0.14 & 0.39 & 0.73 & 0.75 & 0.71 & 0.69 \\
\hline \multirow{2}{*}{ WR NMF[14] } & PESQ & 1.54 & 1.74 & 2.01 & 2.61 & 2.7 & 2.88 \\
\hline & $\triangle$ PESQ & 0.19 & 0.29 & 0.68 & 0.69 & 0.62 & 0.61 \\
\hline \multirow{2}{*}{ NR NMF[14] } & PESQ & 1.52 & 1.7 & 1.84 & 2.48 & 2.6 & 2.76 \\
\hline & $\triangle$ PESQ & 0.17 & 0.25 & 0.51 & 0.56 & 0.52 & 0.49 \\
\hline \multirow{2}{*}{ PR NMF[11] } & PESQ & 1.5 & 1.6 & 1.75 & 2.28 & 2.46 & 2.61 \\
\hline & $\Delta$ PESQ & 0.15 & 0.15 & 0.42 & 0.36 & 0.38 & 0.34 \\
\hline \multirow{2}{*}{ HRNR-SPU[13] } & PESQ & 1.46 & 1.68 & 1.86 & 2.45 & 2.56 & 2.7 \\
\hline & $\Delta$ PESQ & 0.11 & 0.23 & 0.53 & 0.53 & 0.48 & 0.43 \\
\hline \multirow{2}{*}{ HRNR[5] } & PESQ & 1.44 & 1.62 & 1.78 & 2.35 & 2.45 & 2.61 \\
\hline & $\triangle$ PESQ & 0.09 & 0.17 & 0.45 & 0.43 & 0.37 & 0.34 \\
\hline
\end{tabular}

In table 3 , the comparison of average PESQ, $\triangle \mathrm{PESQ}$ performance measures for the 30 speech signals corrupted by white noise under input SNRs of $-10 \mathrm{~dB},-5 \mathrm{~dB}, 0 \mathrm{~dB}, 5$ $\mathrm{dB}, 10 \mathrm{~dB}, 15 \mathrm{~dB}$ is listed. It is observed that the $\mathrm{SE}+$ CUP-GG + NR-NMF + OU provides improved performance than all compared methods and it provides a $\triangle \mathrm{PESQ}$ value of 1.18 for the input signal corrupted under white noise at OdB SNR. It is observed that SE+CUP-GG + NMF approach provides an $\triangle \mathrm{PESQ}$ of 1.16. Also it is observed that CUP-GG estimator provides an improved $\triangle \mathrm{PESQ}$ value of 0.92 compared to posterior WR-NMF of 0.68 at $0 \mathrm{~dB}$ input SNR. It is noted that the Bayesian estimators exhibited comparable improvement under low SNRs than to traditional NMF approach. Also noted that combination of Bayesian estimator with NMF approach provides improved results in case of white noise.

In table 4, the Comparison of Average STOI, \%STOI performance measures for the 30 speech signals corrupted by White noise under Input SNRs of $-10 \mathrm{~dB},-5 \mathrm{~dB}, 0 \mathrm{~dB}$,
$5 \mathrm{~dB}, 10 \mathrm{~dB}, 15 \mathrm{~dB}$ is listed. It is observed that \% STOI improvement of STOI values for SE + CUP - GG + NR $\mathrm{NMF}+\mathrm{OU}$ approach is 7.15, 6.04, 3.76, 2.64, 2.04, 1.12 and for CUP-GG estimator is 5.21, 4.87, 3.45, 1.99, 1.57, 0.67 and for WR-NMF approach is 2.91, 2.62, 2.23, 1.09, $0.87,0.25$ at input SNRs of $-10 \mathrm{~dB},-5 \mathrm{~dB}, 0 \mathrm{~dB}, 5 \mathrm{~dB}, 10$ $\mathrm{dB}, 15 \mathrm{~dB}$ respectively. It is noted that at $-10 \mathrm{~dB}$ and -5 $\mathrm{dB}$ SNRs there is significant improvement in SE+CUPGG+NMF based approaches than to all other methods.

In table 5, the Comparison of Average SNR, SDR, Seg SNR performance measures for the 30 speech signals corrupted by White noise under input SNRs of $-10 \mathrm{~dB}$, $5 \mathrm{~dB}, 0 \mathrm{~dB}, 5 \mathrm{~dB}, 10 \mathrm{~dB}, 15 \mathrm{~dB}$ is listed. The proposed method SE+CUP-GG+NR-NMF+OU provides SNR values of 4.81, 6.94, 9.34, 15.79, 19.04, 23.38 and that for SE+CUP-GG+NMF approach are $4.21,6.83,8.15,14.21$, 17.97, 23.12. The NR-NMF approach provides SNR values of $-1.34,1.78,7.23,13.87,17.68$ and 22.34 .

Table 4. Comparison of Average STOI, \% STOI performance measures for the 30 speech signals corrupted by white noise under Input SNRs of -10 B, $-5 \mathrm{~dB}, 0 \mathrm{~dB}, 5 \mathrm{~dB}, 10 \mathrm{~dB}, 15 \mathrm{~dB}$

\begin{tabular}{|c|c|c|c|c|c|c|c|}
\hline Input SNR in dB & $\begin{array}{c}\text { Performance } \\
\text { Measure }\end{array}$ & $\mathbf{- 1 0}$ & $\mathbf{- 5}$ & $\mathbf{0}$ & $\mathbf{5}$ & $\mathbf{1 0}$ \\
\hline Wethod & STOI & 0.4602 & 0.5902 & 0.7273 & 0.7816 & 0.9296 \\
\hline Without Processing & \% STOI & 7.15 & 6.04 & 3.76 & 2.64 & 2.04 \\
\hline NMF+GG+NR- & STOI & 0.4885 & 0.6243 & 0.7525 & 0.8013 & 0.9462 & 0.9509 \\
\cline { 2 - 7 } NE+CUP-GG+WR- & \% STOI & 6.16 & 5.78 & 3.47 & 2.52 & 1.79 & 0.79 \\
\cline { 2 - 7 } NMF+OU & STOI & 0.4885 & 0.6243 & 0.7525 & 0.8013 & 0.9462 & 0.9509 \\
\hline
\end{tabular}




\begin{tabular}{|c|c|c|c|c|c|c|c|}
\hline \multirow{2}{*}{$\mathrm{SE}+\mathrm{CUP}-\mathrm{GG}+\boldsymbol{\beta}-\mathrm{NMF}+\mathrm{OU}$} & \% STOI & 6.23 & 5.76 & 3.52 & 2.43 & 1.87 & 0.78 \\
\hline & STOI & 0.4889 & 0.6242 & 0.7529 & 0.8006 & 0.9469 & 0.9508 \\
\hline \multirow{2}{*}{ SE+CUP GG+NMF[9] } & \% STOI & 7.12 & 5.97 & 3.68 & 2.56 & 1.98 & 0.97 \\
\hline & STOI & 0.493 & 0.6254 & 0.7541 & 0.8016 & 0.9480 & 0.9527 \\
\hline \multirow{2}{*}{$\mathrm{NMF}+\mathrm{OU}[12]$} & \% STOI & 4.25 & 3.35 & 3.24 & 1.32 & 0.89 & 0.75 \\
\hline & STOI & 0.4798 & 0.61 & 0.7509 & 0.7919 & 0.9378 & 0.9506 \\
\hline \multirow{2}{*}{ CUP-GG[9] } & \% STOI & 5.21 & 4.87 & 3.45 & 1.99 & 1.57 & 0.67 \\
\hline & STOI & 0.4842 & 0.6189 & 0.748 & 0.7971 & 0.9441 & 0.9498 \\
\hline \multirow{2}{*}{ CUP-NG[9] } & \% STOI & 4.76 & 3.95 & 3.46 & 1.45 & 1.32 & 0.87 \\
\hline & STOI & 0.4821 & 0.6135 & 0.7525 & 0.7929 & 0.9418 & 0.9517 \\
\hline \multirow{2}{*}{ CUP[8] } & \% STOI & 3.78 & 3.05 & 2.89 & 0.98 & 0.69 & 0.47 \\
\hline & STOI & 0.4623 & 0.5937 & 0.7326 & 0.7877 & 0.9382 & 0.9524 \\
\hline \multirow{2}{*}{ WR NMF[14] } & \% STOI & 2.91 & 2.62 & 2.23 & 1.09 & 0.87 & 0.25 \\
\hline & STOI & 0.4736 & 0.6057 & 0.7435 & 0.7901 & 0.9376 & 0.9458 \\
\hline \multirow{2}{*}{ NR NMF[14] } & $\%$ STOI & 2.89 & 2.47 & 2.12 & 1.14 & 0.98 & 0.23 \\
\hline & STOI & 0.4735 & 0.6048 & 0.7427 & 0.7905 & 0.9387 & 0.9456 \\
\hline \multirow{2}{*}{ PR NMF[11] } & \% STOI & 1.82 & 1.52 & 1.28 & 0.47 & 0.35 & 0.26 \\
\hline & STOI & 0.4686 & 0.5992 & 0.7366 & 0.7852 & 0.9328 & 0.9459 \\
\hline \multirow{2}{*}{ HRNR-SPU[13] } & \% STOI & -1.9 & -1.62 & -1.12 & -1.45 & -1.02 & -0.92 \\
\hline & STOI & 0.4515 & 0.5806 & 0.7192 & 0.7702 & 0.9201 & 0.9348 \\
\hline \multirow{2}{*}{ HRNR[5] } & \% STOI & -2.21 & -1.76 & -1.27 & -1.56 & -1.18 & -1.03 \\
\hline & STOI & 0.45 & 0.5798 & 0.7181 & 0.7694 & 0.9186 & 0.9337 \\
\hline
\end{tabular}

Table 5. Comparison of Average SNR, SDR, Segmental SNR performance measures for the 30 speech signals corrupted by white noise under Input SNRs of $-10 \mathrm{~dB},-5 \mathrm{~dB}, 0 \mathrm{~dB}, 5 \mathrm{~dB}, 10 \mathrm{~dB}, 15 \mathrm{~dB}$

\begin{tabular}{|c|c|c|c|c|c|c|c|}
\hline Input SNR in dB & $\begin{array}{l}\text { Performance } \\
\text { Measure }\end{array}$ & -10 & -5 & $\mathbf{0}$ & 5 & 10 & 15 \\
\hline \multirow{3}{*}{$\mathrm{SE}+\mathrm{CUP}-\mathrm{GG}+\mathrm{NR}-\mathrm{NMF}+\mathrm{OU}$} & SNR & 4.81 & 6.94 & 9.34 & 15.79 & 19.04 & 23.38 \\
\hline & SDR & 4.5 & 4.8 & 9.4 & 15.2 & 18.5 & 22.5 \\
\hline & Seg SNR & 3.79 & 5.94 & 7.73 & 12.23 & 16.13 & 20.78 \\
\hline \multirow{3}{*}{$\mathrm{SE}+\mathrm{CUP}-\mathrm{GG}+\mathrm{WR}-\mathrm{NMF}+\mathrm{OU}$} & SNR & 4.62 & 6.91 & 9.15 & 15.43 & 18.63 & 23.45 \\
\hline & SDR & 4.68 & 5.1 & 9.2 & 14.8 & 18.3 & 22.2 \\
\hline & Seg SNR & 3.83 & 5.89 & 7.58 & 11.98 & 15.95 & 20.14 \\
\hline \multirow{3}{*}{$\mathrm{SE}+\mathrm{CUP}-\mathrm{GG}+\boldsymbol{\beta}-\mathrm{NMF}+\mathrm{OU}$} & SNR & 4.53 & 6.93 & 8.76 & 14.65 & 18.12 & 23.09 \\
\hline & SDR & 3.9 & 5.4 & $8 . .9$ & 14.2 & 17.9 & 22.6 \\
\hline & Seg SNR & 3.71 & 5.78 & 7.25 & 11.76 & 15.42 & 19.93 \\
\hline \multirow{3}{*}{ SE+CUP GG+NMF[9] } & SNR & 4.21 & 6.83 & 8.15 & 14.21 & 17.97 & 23.12 \\
\hline & SDR & 3.4 & 5.3 & 8.6 & $13 . .8$ & 17.5 & 22.2 \\
\hline & Seg SNR & 3.81 & 5.98 & 7.12 & 11.54 & 15.23 & 19.87 \\
\hline \multirow{3}{*}{$\mathrm{NMF}+\mathrm{OU}[12]$} & SNR & 2.51 & 4.69 & 6.58 & 12.62 & 16.54 & 20.56 \\
\hline & SDR & 1.8 & 3.68 & 5.69 & 11.53 & 15.69 & 19.56 \\
\hline & Seg SNR & 1.32 & 3.05 & 4.95 & 10.25 & 14.41 & 18.36 \\
\hline \multirow{3}{*}{ CUP-GG[9] } & SNR & 3.97 & 6.13 & 7.95 & 13.87 & 17.89 & 22.68 \\
\hline & SDR & 2.9 & 4.8 & 7.9 & 12.1 & 16.6 & 21.6 \\
\hline & Seg SNR & 3.67 & 5.87 & 6.98 & 10.98 & 14.98 & 19.11 \\
\hline \multirow{3}{*}{ CUP-NG[9] } & SNR & 2.96 & 5.02 & 7.98 & 13.21 & 17.69 & 22.87 \\
\hline & SDR & 2.3 & 4.4 & 7.1 & 11.8 & 16.1 & 21.2 \\
\hline & Seg SNR & 2.29 & 4.87 & 6.92 & 10.77 & 14.67 & 19.03 \\
\hline \multirow{3}{*}{ CUP $[8]$} & SNR & 2.36 & 4.89 & 7.23 & 12.15 & 16.94 & 21.98 \\
\hline & SDR & 1.9 & 4.2 & 6.8 & 11.65 & 15.6 & 19.5 \\
\hline & Seg SNR & 1.83 & 3.12 & 6.36 & 10.23 & 14.76 & 18.78 \\
\hline \multirow{3}{*}{ NR-NMF[14] } & SNR & -1.34 & 1.78 & 7.23 & 13.87 & 17.68 & 22.34 \\
\hline & SDR & -1.8 & -1.1 & 6.8 & 12.7 & 16.6 & 19.8 \\
\hline & Seg SNR & -1.98 & -1.32 & 4.78 & 9.02 & 15.76 & 18.79 \\
\hline \multirow{3}{*}{ WR-NMF[14] } & SNR & -1.63 & 1.35 & 6.42 & 11.37 & 17.02 & 22.21 \\
\hline & SDR & -1.8 & -0.6 & 5.5 & 11.6 & 15.8 & 21 \\
\hline & Seg SNR & -2.32 & -1.42 & 4.03 & 8.42 & 14.15 & 18.95 \\
\hline \multirow{3}{*}{ PR NMF [11] } & SNR & -1.91 & 1.23 & 4.42 & 9.34 & 15.12 & 21.32 \\
\hline & SDR & -2.1 & $\begin{array}{l}-1.1 \\
\end{array}$ & 4.2 & 8.9 & 13.9 & 19.5 \\
\hline & Seg SNR & -2.7 & -1.47 & 3.14 & 7.21 & 12.43 & 17.52 \\
\hline \multirow{3}{*}{ HRNR-SPU [13] } & SNR & 1.98 & 1.89 & 4.32 & 9.12 & 14.46 & 19.76 \\
\hline & SDR & 1.24 & 1.68 & 2.67 & 8.1 & 13.4 & 18.2 \\
\hline & Seg SNR & 0.34 & 1.34 & 2.12 & 6.32 & 11.68 & 17.87 \\
\hline \multirow{3}{*}{ HRNR[5] } & SNR & 1.21 & 1.78 & 2.62 & 7.98 & 14.23 & 18.65 \\
\hline & SDR & 0.94 & 1.54 & 2.56 & 7.6 & 12.3 & 17.2 \\
\hline & Seg SNR & 0.13 & 1.02 & 1.78 & 5.15 & 10.69 & 16.56 \\
\hline
\end{tabular}


Table 6. .Comparison of Average PESQ, $\triangle \mathrm{PESQ}$ performance measures for the 30 speech signals corrupted by Babble noise under Input SNRs of -10 $\mathrm{dB},-5 \mathrm{~dB}, 0 \mathrm{~dB}, 5 \mathrm{~dB}, 10 \mathrm{~dB}, 15 \mathrm{~dB}$

\begin{tabular}{|c|c|c|c|c|c|c|c|}
\hline $\begin{array}{l}\text { Input SNR in dB } \\
\text { Method }\end{array}$ & $\begin{array}{c}\text { Performance } \\
\text { Measure }\end{array}$ & -10 & -5 & $\mathbf{0}$ & 5 & 10 & 15 \\
\hline Without Processing & PESQ & 1.24 & 1.35 & 1.79 & 2.11 & 2.41 & 2.58 \\
\hline \multirow{2}{*}{$\mathrm{SE}+\mathrm{CUP}-\mathrm{GG}+\mathrm{NR}-\mathrm{NMF}+\mathrm{OU}$} & PESQ & 1.53 & 1.78 & 2.61 & 2.9 & 3.16 & 3.3 \\
\hline & $\triangle \mathrm{PESQ}$ & 0.29 & 0.43 & 0.82 & 0.79 & 0.75 & 0.72 \\
\hline \multirow{2}{*}{$\mathrm{SE}+\mathrm{CUP}-\mathrm{GG}+\mathrm{WR}-\mathrm{NMF}+\mathrm{OU}$} & PESQ & 1.49 & 1.74 & 2.5 & 2.86 & 3.13 & 3.27 \\
\hline & $\Delta$ PESQ & 0.25 & 0.39 & 0.71 & 0.75 & 0.72 & 0.69 \\
\hline \multirow{2}{*}{$\mathrm{SE}+\mathrm{CUP}-\mathrm{GG}+\boldsymbol{\beta}-\mathrm{NMF}+\mathrm{OU}$} & PESQ & 1.24 & 1.73 & 2.44 & 2.8 & 3.06 & 3.22 \\
\hline & $\triangle \mathrm{PESQ}$ & 0.26 & 0.38 & 0.65 & 0.69 & 0.65 & 0.64 \\
\hline \multirow{2}{*}{ SE+CUP GG+NMF[9] } & PESQ & 1.45 & 1.69 & 2.41 & 2.76 & 3.07 & 3.22 \\
\hline & $\triangle \mathrm{PESQ}$ & 0.21 & 0.34 & 0.62 & 0.65 & 0.66 & 0.64 \\
\hline \multirow{2}{*}{$\mathrm{NMF}+\mathrm{OU}[12]$} & PESQ & 1.32 & 1.53 & 2.26 & 2.54 & 2.89 & 2.97 \\
\hline & $\triangle$ PESQ & 0.08 & 0.18 & 0.47 & 0.43 & 0.48 & 0.39 \\
\hline \multirow{2}{*}{ CUP-GG[9] } & PESQ & 1.41 & 1.63 & 2.36 & 2.69 & 3 & 3.11 \\
\hline & $\triangle$ PESQ & 0.17 & 0.28 & 0.57 & 0.58 & 0.59 & 0.53 \\
\hline \multirow{2}{*}{ CUP-NG[9] } & PESQ & 1.37 & 1.61 & 2.26 & 2.65 & 2.97 & 3.08 \\
\hline & $\triangle$ PESQ & 0.13 & 0.26 & 0.47 & 0.54 & 0.56 & 0.5 \\
\hline \multirow{2}{*}{ CUP[8] } & PESQ & 1.31 & 1.5 & 2.2 & 2.55 & 2.88 & 3 \\
\hline & $\Delta$ PESQ & 0.07 & 0.15 & 0.41 & 0.44 & 0.47 & 0.42 \\
\hline \multirow{2}{*}{ WR NMF[14] } & PESQ & 1.41 & 1.61 & 2.24 & 2.63 & 2.91 & 3.06 \\
\hline & $\triangle \mathrm{PESQ}$ & 0.17 & 0.26 & 0.45 & 0.52 & 0.5 & 0.48 \\
\hline \multirow{2}{*}{ NR NMF[14] } & PESQ & 1.36 & 1.57 & 2.22 & 2.61 & 2.89 & 3.04 \\
\hline & $\triangle$ PESQ & 0.12 & 0.22 & 0.43 & 0.5 & 0.48 & 0.46 \\
\hline \multirow{2}{*}{ PR NMF[11] } & PESQ & 1.34 & 1.53 & 2.17 & 2.54 & 2.83 & 2.97 \\
\hline & $\Delta$ PESQ & 0.1 & 0.18 & 0.38 & 0.43 & 0.42 & 0.39 \\
\hline \multirow{2}{*}{ HRNR-SPU[13] } & PESQ & 1.32 & 1.53 & 2.16 & 2.45 & 2.73 & 2.84 \\
\hline & $\triangle$ PESQ & 0.08 & 0.18 & 0.37 & 0.34 & 0.32 & 0.26 \\
\hline \multirow{2}{*}{ HRNR[5] } & PESQ & 1.27 & 1.5 & 2.1 & 2.4 & 2.68 & 2.77 \\
\hline & $\triangle \mathrm{PESQ}$ & 0.03 & 0.15 & 0.31 & 0.29 & 0.27 & 0.19 \\
\hline
\end{tabular}

In table 6 , the Comparison of Average PESQ, $\triangle \mathrm{PESQ}$ performance measures for the 30 speech signals corrupted by Babble noise under input SNRs of $-10 \mathrm{~dB},-5 \mathrm{~dB}, 0 \mathrm{~dB}$, $5 \mathrm{~dB}, 10 \mathrm{~dB}, 15 \mathrm{~dB}$ is listed. It is observed that the, $\mathrm{SE}+$ CUP-GG + NR-NMF + OU provides improved performance than to all compared methods and it provides a $\triangle \mathrm{PESQ}$ value of 0.82 for the input signal corrupted under Babble noise at OdB SNR. It is observed that SE+ CUP-GG + NMF approach provides an PESQ improvement of 0.62. Also it is observed that CUP-GG estimators provides an improved $\triangle \mathrm{PESQ}$ value of 0.57 compared to posterior WR-NMF of 0.45 at $0 \mathrm{~dB}$ input SNR. It is noted that the Bayesian estimators shown comparable improvement under low SNRs than to traditional NMF approach. Also noted that combination of Bayesian estimator with NMF approach provides improved results in case of Babble noise.

In table 7, the Comparison of Average STOI, \%STOI performance measures for the 30 speech signals corrupted by Babble noise under Input SNRs of $-10 \mathrm{~dB},-5 \mathrm{~dB}, 0 \mathrm{~dB}$, $5 \mathrm{~dB}, 10 \mathrm{~dB}$,

$15 \mathrm{~dB}$ is listed. It is observed that \% STOI improvement of STOI values for SE + CUP $-\mathrm{GG}+\mathrm{NR}$ $\mathrm{NMF}+\mathrm{OU}$ approach is $3.22,4.36,3.15,2.21,1.66,0.54$ and for CUP-GG estimator is $-0.02,-0.01,0.09,0.06$, $0.05,0.03$ and for WR-NMF approach is $1.32,1.13,0.78$, $0.65,0.34,0.12$ at input SNRs of $-10 \mathrm{~dB},-5 \mathrm{~dB}, 0 \mathrm{~dB}, 5$ $\mathrm{dB}, 10 \mathrm{~dB}, 15 \mathrm{~dB}$ respectively. It is noted that at $-10 \mathrm{~dB}$, $5 \mathrm{~dB}$ SNRs there is significant improvement in SE+CUPGG+NMF based approaches than to all other methods.

In table 8, the Comparison of Average SNR, SDR, Seg SNR performance measures for the 30 speech signals corrupted by Babble noise under Input SNRs of $-10 \mathrm{~dB}$, $5 \mathrm{~dB}, 0 \mathrm{~dB}, 5 \mathrm{~dB}, 10 \mathrm{~dB}, 15 \mathrm{~dB}$ is listed. The proposed method SE+CUP-GG+NR-NMF+OU provides an SNR of $4.35,7.65,10.06,11.86,15.86,20.84$ and for $\mathrm{SE}+\mathrm{CUP}-\mathrm{GG}+\mathrm{NMF}$ approach is $3.56,6.21,8.23,9.65$, $12.58,18.43$. The NR-NMF approach provides an SNR of $1.56,3.67,6.21,8.68,13.12$, and 15.85

Table 7. Comparison of Average STOI, \% STOI performance measures for the 30 speech signals corrupted by Babble noise under Input SNRs of -10 $\mathrm{dB},-5 \mathrm{~dB}, 0 \mathrm{~dB}, 5 \mathrm{~dB}, 10 \mathrm{~dB}, 15 \mathrm{~dB}$

\begin{tabular}{|c|c|c|c|c|c|c|c|}
\hline $\begin{array}{l}\text { Input SNR in dB } \\
\text { Method }\end{array}$ & $\begin{array}{l}\text { Performance } \\
\text { Measure }\end{array}$ & -10 & -5 & $\mathbf{0}$ & 5 & 10 & 15 \\
\hline Without Processing & STOI & 0.3352 & 0.5612 & 0.6186 & 0.7761 & 0.8629 & 0.9336 \\
\hline \multirow{2}{*}{$\begin{array}{c}\mathrm{SE}+\mathrm{CUP}-\mathrm{GG}+\mathrm{NR}- \\
\mathrm{NMF}+\mathrm{OU}\end{array}$} & $\%$ STOI & 3.22 & 4.36 & 3.15 & 2.21 & 1.66 & 0.54 \\
\hline & STOI & 0.346 & 0.5857 & 0.6381 & 0.7932 & 0.8772 & 0.9386 \\
\hline \multirow{2}{*}{$\begin{array}{c}\text { SE+CUP-GG+WR- } \\
\text { NMF+OU }\end{array}$} & $\%$ STOI & 3.18 & 4.32 & 3.18 & 2.16 & 1.68 & 0.59 \\
\hline & STOI & 0.3458 & 0.5854 & 0.6383 & 0.7929 & 0.8774 & 0.9391 \\
\hline \multirow{2}{*}{$\mathrm{SE}+\mathrm{CUP}-\mathrm{GG}+\boldsymbol{\beta}-\mathrm{NMF}+\mathrm{OU}$} & $\%$ STOI & 3.14 & 4.24 & 3.12 & 2.11 & 1.66 & 0.61 \\
\hline & STOI & 0.3457 & 0.585 & 0.6379 & 0.7925 & 0.8772 & 0.9393 \\
\hline
\end{tabular}




\begin{tabular}{|c|c|c|c|c|c|c|c|}
\cline { 2 - 7 } SE+CUP GG+NMF[9] & \% STOI & 1.98 & 1.45 & 1.37 & 1.41 & 0.96 & 0.65 \\
\cline { 2 - 8 } & STOI & 0.3418 & 0.5693 & 0.6271 & 0.7870 & 0.8712 & 0.9397 \\
\hline \multirow{2}{*}{ NMF+OU[12] } & \% STOI & 1.87 & 1.23 & 1.11 & 1.01 & 0.89 & 0.57 \\
\cline { 2 - 8 } & STOI & 0.3415 & 0.5681 & 0.6255 & 0.7839 & 0.8706 & 0.9389 \\
\hline \multirow{2}{*}{ CUP-GG[9] } & \% STOI & -0.02 & -0.01 & 0.09 & 0.06 & 0.05 & 0.03 \\
\cline { 2 - 8 } & STOI & 0.3351 & 0.5611 & 0.6192 & 0.7766 & 0.8633 & 0.9339 \\
\hline \multirow{2}{*}{ CUP-NG[9] } & \% STOI & -0.35 & -0.19 & -0.01 & 0.37 & 0.24 & 0.01 \\
\cline { 2 - 8 } & STOI & 0.3340 & 0.5601 & 0.6185 & 0.7789 & 0.8649 & 0.9337 \\
\hline \multirow{2}{*}{ CUP[8] } & \% STOI & -1.09 & -0.62 & -0.17 & 0.25 & 0.15 & 0.02 \\
\cline { 2 - 8 } & STOI & 0.3315 & 0.5577 & 0.6176 & 0.7780 & 0.8642 & 0.9338 \\
\hline \multirow{2}{*}{ WR NMF[14] } & \% STOI & 1.32 & 1.13 & 0.78 & 0.65 & 0.34 & 0.12 \\
\cline { 2 - 8 } & STOI & 0.3396 & 0.5675 & 0.6234 & 0.7811 & 0.8658 & 0.9347 \\
\hline \multirow{2}{*}{ NR NMF[14] } & \% STOI & 1.23 & 1.11 & 0.72 & 0.58 & 0.24 & 0.09 \\
\cline { 2 - 8 } & STOI & 0.3393 & 0.5674 & 0.6231 & 0.7806 & 0.865 & 0.9344 \\
\hline \multirow{2}{*}{ PR NMF[11] } & \% STOI & 0.98 & 0.87 & 0.67 & 0.21 & 0.11 & 0.08 \\
\cline { 2 - 8 } & STOI & 0.3385 & 0.5661 & 0.6227 & 0.7777 & 0.8638 & 0.9343 \\
\hline \multirow{2}{*}{ HRNR-SPU[13] } & \% STOI & -2.12 & -1.96 & -1.33 & -1.61 & -1.18 & -1.15 \\
\cline { 2 - 8 } & STOI & 0.3281 & 0.5502 & 0.6104 & 0.7636 & 0.8527 & 0.9229 \\
\hline \multirow{2}{*}{ HRNR[5] } & \% STOI & -2.67 & -2.13 & -1.98 & -1.79 & -1.27 & -1.21 \\
\cline { 2 - 8 } & STOI & 0.3262 & 0.5492 & 0.6063 & 0.7622 & 0.8519 & 0.9223 \\
\hline
\end{tabular}

Table 8.Comparison of Average STOI, \% STOI performance measures for the 30 speech signals corrupted by Babble noise under Input SNRs of -10 $\mathrm{dB},-5 \mathrm{~dB}, 0 \mathrm{~dB}, 5 \mathrm{~dB}, 10 \mathrm{~dB}, 15 \mathrm{~dB}$

\begin{tabular}{|c|c|c|c|c|c|c|c|}
\hline Method Input SNR in dB & $\begin{array}{l}\text { Performance } \\
\text { Measure }\end{array}$ & -10 & -5 & $\mathbf{0}$ & 5 & 10 & 15 \\
\hline \multirow{3}{*}{ SE+CUP-GG+NR-NMF+OU } & SNR & 4.35 & 7.65 & 10.06 & 11.86 & 15.86 & 20.84 \\
\hline & SDR & 4.02 & 6.45 & 8.98 & 10.25 & 13.62 & 18.21 \\
\hline & Seg SNR & 3.62 & 5.85 & 7.68 & 9.75 & 12.78 & 16.54 \\
\hline \multirow{3}{*}{ SE+CUP-GG+WR-NMF+OU } & SNR & 4.12 & 6.34 & 8.78 & 10.12 & 13.48 & 19.78 \\
\hline & SDR & 3.56 & 5.87 & 8.84 & 10.14 & 13.54 & 18.03 \\
\hline & Seg SNR & 3.45 & 5.68 & 7.65 & 9.94 & 12.86 & 16.23 \\
\hline \multirow{3}{*}{$\mathrm{SE}+\mathrm{CUP}-\mathrm{GG}+\boldsymbol{\beta}-\mathrm{NMF}+\mathrm{OU}$} & SNR & 4.34 & 6.89 & 8.9 & 10.23 & 13.65 & 18.66 \\
\hline & SDR & 3.45 & 5.76 & 8.56 & 9.89 & 13.27 & 17.76 \\
\hline & Seg SNR & 3.26 & 5.69 & 7.35 & 9.85 & 12.82 & 15.87 \\
\hline \multirow{3}{*}{ SE+CUP GG+NMF[9] } & SNR & 3.56 & 6.21 & 8.23 & 9.65 & 12.58 & 18.43 \\
\hline & SDR & 3.23 & 6.24 & 8.41 & 9.75 & 12.98 & 17.54 \\
\hline & Seg SNR & 3.03 & 5.43 & 6.92 & 9.17 & 12.36 & 15.38 \\
\hline \multirow{3}{*}{$\mathrm{NMF}+\mathrm{OU}[12]$} & SNR & 3.16 & 3.98 & 7.45 & 8.89 & 12.95 & 17.54 \\
\hline & SDR & 2.87 & 3.24 & 7.48 & 8.62 & 12.23 & 14.85 \\
\hline & Seg SNR & 2.54 & 4.21 & 6.21 & 8.17 & 11.56 & 13.89 \\
\hline \multirow{3}{*}{ CUP-GG[9] } & SNR & 3.11 & 5.98 & 7.95 & 9.12 & 12.24 & 17.98 \\
\hline & SDR & 2.97 & 3.56 & 7.79 & 8.94 & 12.86 & 15.74 \\
\hline & Seg SNR & 2.98 & 5.11 & 6.8 & 8.97 & 12.11 & 14.65 \\
\hline \multirow{3}{*}{ CUP-NG[9] } & SNR & 3.15 & 4.56 & 7.89 & 9.65 & 13.14 & 17.85 \\
\hline & SDR & 2.98 & 3.38 & 7.56 & 8.89 & 12.78 & 15.56 \\
\hline & Seg SNR & 2.19 & 4.11 & 6.45 & 8.42 & 11.43 & 13.71 \\
\hline \multirow{3}{*}{ CUP[8] } & SNR & 3.05 & 3.45 & 7.65 & 9.24 & 12.89 & 17.14 \\
\hline & SDR & 2.76 & 3.15 & 7.11 & 8.56 & 12.43 & 15.13 \\
\hline & Seg SNR & 1.64 & 2.35 & 5.97 & 8.15 & 11.32 & 13.57 \\
\hline \multirow{3}{*}{ NR-NMF[14] } & SNR & 1.56 & 3.67 & 6.21 & 8.68 & 13.12 & 15.85 \\
\hline & SDR & 0.76 & 2.46 & 5.67 & 7.89 & 12.03 & 14.87 \\
\hline & Seg SNR & -2.67 & -0.85 & 3.02 & 6.51 & 10.65 & 13.21 \\
\hline \multirow{3}{*}{ WR-NMF[14] } & SNR & 1.24 & 3.58 & 5.87 & 8.76 & 12.98 & 15.53 \\
\hline & SDR & 0.16 & 2.14 & 5.45 & 7.57 & 12.24 & 14.98 \\
\hline & Seg SNR & -3.22 & -1.28 & 2.45 & 5.08 & 10.45 & 13.01 \\
\hline \multirow{3}{*}{ PR NMF [11] } & SNR & 0.08 & 2.17 & 4.23 & 7.52 & 11.34 & 14.25 \\
\hline & SDR & -2.31 & 1.23 & 3.67 & 7.59 & 10.89 & 12.97 \\
\hline & Seg SNR & -3.56 & -1.54 & 2.12 & 4.87 & 9.45 & 12.02 \\
\hline \multirow{3}{*}{ HRNR-SPU [13] } & SNR & -4.12 & -1.34 & -1.21 & 5.21 & 11.54 & 12.89 \\
\hline & SDR & -6.54 & -3.85 & -0.98 & 2.98 & 6.24 & 12.25 \\
\hline & Seg SNR & -9.03 & -3.62 & -1.32 & 2.78 & 6.87 & 9.85 \\
\hline \multirow{3}{*}{ HRNR[5] } & SNR & -5.42 & -1.98 & -0.12 & 5.36 & 10.59 & 12.21 \\
\hline & SDR & -7.45 & -2.76 & -0.28 & 2.69 & 9.87 & 11.41 \\
\hline & Seg SNR & -9.82 & -4.51 & -1.41 & 2.05 & 5.87 & 9.14 \\
\hline
\end{tabular}


Table 9.Comparison of Average PESQ, $\triangle \mathrm{PESQ}$ performance measures for the 30 speech signals corrupted by Pink noise under Input SNRs of -10 dB, $-5 \mathrm{~dB}, 0 \mathrm{~dB}, 5 \mathrm{~dB}, 10 \mathrm{~dB}, 15 \mathrm{~dB}$

\begin{tabular}{|c|c|c|c|c|c|c|c|}
\hline $\begin{array}{l}\text { Input SNR in dB } \\
\text { Method }\end{array}$ & $\begin{array}{l}\text { Performance } \\
\text { Measure }\end{array}$ & -10 & -5 & $\mathbf{0}$ & 5 & 10 & 15 \\
\hline Without Processing & PESQ & 1.13 & 1.43 & 1.8 & 2.05 & 2.27 & 2.55 \\
\hline \multirow{2}{*}{$\mathrm{SE}+\mathrm{CUP}-\mathrm{GG}+\mathrm{NR}-\mathrm{NMF}+\mathrm{OU}$} & PESQ & 1.42 & 2.22 & 2.82 & 3.01 & 3.15 & 3.36 \\
\hline & $\triangle \mathrm{PESQ}$ & 0.29 & 0.79 & 1.02 & 0.96 & 0.88 & 0.81 \\
\hline \multirow{2}{*}{ SE+CUP-GG+WR-NMF+OU } & PESQ & 1.4 & 2.05 & 2.77 & 2.97 & 3.13 & 3.34 \\
\hline & $\triangle \mathrm{PESQ}$ & 0.27 & 0.62 & 0.97 & 0.92 & 0.86 & 0.79 \\
\hline \multirow{2}{*}{$\mathrm{SE}+\mathrm{CUP}-\mathrm{GG}+\boldsymbol{\beta}-\mathrm{NMF}+\mathrm{OU}$} & PESQ & 1.38 & 1.99 & 2.76 & 3 & 3.2 & 3.37 \\
\hline & $\triangle \mathrm{PESQ}$ & 0.25 & 0.56 & 0.96 & 0.95 & 0.93 & 0.82 \\
\hline \multirow{2}{*}{ SE+CUP GG+NMF[9] } & PESQ & 1.35 & 1.92 & 2.64 & 2.87 & 3.09 & 3.34 \\
\hline & $\triangle \mathrm{PESQ}$ & 0.22 & 0.49 & 0.84 & 0.82 & 0.82 & 0.79 \\
\hline \multirow{2}{*}{$\mathrm{NMF}+\mathrm{OU}[12]$} & PESQ & 1.25 & 1.8 & 2.47 & 2.7 & 2.9 & 3.15 \\
\hline & $\triangle \mathrm{PESQ}$ & 0.12 & 0.37 & 0.67 & 0.65 & 0.63 & 0.6 \\
\hline \multirow{2}{*}{ CUP-GG[9] } & PESQ & 1.3 & 1.86 & 2.59 & 2.83 & 3.03 & 3.2 \\
\hline & $\triangle \mathrm{PESQ}$ & 0.17 & 0.43 & 0.79 & 0.78 & 0.76 & 0.65 \\
\hline \multirow{2}{*}{ CUP-NG[9] } & PESQ & 1.27 & 1.84 & 2.54 & 2.78 & 2.97 & 3.19 \\
\hline & $\triangle \mathrm{PESQ}$ & 0.14 & 0.41 & 0.74 & 0.73 & 0.7 & 0.64 \\
\hline \multirow{2}{*}{ CUP[8] } & PESQ & 1.24 & 1.78 & 2.47 & 2.73 & 2.93 & 3.16 \\
\hline & $\triangle \mathrm{PESQ}$ & 0.11 & 0.35 & 0.67 & 0.68 & 0.66 & 0.61 \\
\hline \multirow{2}{*}{ WR NMF[14] } & PESQ & 1.28 & 1.82 & 2.39 & 2.67 & 2.85 & 3.09 \\
\hline & $\triangle \mathrm{PESQ}$ & 0.15 & 0.39 & 0.59 & 0.62 & 0.58 & 0.54 \\
\hline \multirow{2}{*}{ NR NMF[14] } & PESQ & 1.25 & 1.84 & 2.37 & 2.64 & 2.84 & 3.09 \\
\hline & $\triangle \mathrm{PESQ}$ & 0.12 & 0.41 & 0.57 & 0.59 & 0.57 & 0.54 \\
\hline \multirow{2}{*}{ PR NMF[11] } & PESQ & 1.23 & 1.75 & 2.31 & 2.54 & 2.75 & 2.94 \\
\hline & $\triangle \mathrm{PESQ}$ & 0.1 & 0.32 & 0.51 & 0.49 & 0.48 & 0.39 \\
\hline \multirow{2}{*}{ HRNR-SPU[13] } & PESQ & 1.22 & 1.72 & 2.32 & 2.54 & 2.72 & 2.9 \\
\hline & $\triangle \mathrm{PESQ}$ & 0.09 & 0.29 & 0.52 & 0.49 & 0.45 & 0.35 \\
\hline \multirow{2}{*}{ HRNR[5] } & PESQ & 1.17 & 1.62 & 2.21 & 2.45 & 2.63 & 2.84 \\
\hline & $\triangle \mathrm{PESQ}$ & 0.04 & 0.19 & 0.41 & 0.4 & 0.36 & 0.29 \\
\hline
\end{tabular}

In Table 9, the comparison of Average PESQ, $\triangle \mathrm{PESQ}$ performance measures for the 30 speech signals corrupted by Pink noise under input SNRs of $-10 \mathrm{~dB},-5 \mathrm{~dB}, 0 \mathrm{~dB}, 5$ $\mathrm{dB}, 10 \mathrm{~dB}, 15 \mathrm{~dB}$ is listed. It is observed that the, $\mathrm{SE}+$ CUP-GG + NR-NMF + OU provides improved performance than to all compared methods and it provides a $\triangle \mathrm{PESQ}$ value of 1.02 for the input signal corrupted under Pink noise at OdB SNR. It is observed that SE+CUP-GG + NMF approach provides PESQ improvement of 0.84. Also it is observed that CUP-GG estimators provides an improved $\triangle \mathrm{PESQ}$ value of 0.79 compared to posterior WR-NMF of 0.59 at $0 \mathrm{~dB}$ input SNR. It is noted that the Bayesian estimators shown comparable improvement under low SNRs than to traditional NMF approach. Also noted that combination of Bayesian estimator with NMF approach provides improved results in case of Pink noise

In Table 10, the comparison of Average STOI, \%STOI performance measures for the 30 speech signals corrupted by Pink noise under Input SNRs of $-10 \mathrm{~dB},-5 \mathrm{~dB}, 0 \mathrm{~dB}, 5$
$\mathrm{dB}, 10 \mathrm{~dB}, 15 \mathrm{~dB}$ is listed. It is observed that \% STOI improvement of STOI values for SE + CUP - GG + NR $\mathrm{NMF}+\mathrm{OU}$ approach is $7.09,6.23,3.68,2.45,1.78,0.89$ and for CUP-GG estimator is 5.21, 4.87, 3.45, 1.99, 1.57, $0.64 .98,4.69,2.61,1.95,1.46,0.58$ and for WR-NMF approach is $2.73,2.34,2.01,0.93,0.62,0.24$ at input SNRs of $-10 \mathrm{~dB},-5 \mathrm{~dB}, 0 \mathrm{~dB}, 5 \mathrm{~dB}, 10 \mathrm{~dB}, 15 \mathrm{~dB}$ respectively. It is noted that at $-10 \mathrm{~d},-5 \mathrm{~dB}$ SNRs there is significant improvement in SE+CUP-GG+NMF based approaches than to all other methods.

In table 11, the Comparison of Average SNR, SDR, Seg SNR performance measures for the 30 speech signals corrupted by Pink noise under Input SNRs of -10 dB, $5 \mathrm{~dB}, 0 \mathrm{~dB}, 5 \mathrm{~dB}, 10 \mathrm{~dB}, 15 \mathrm{~dB}$ is listed. The proposed method SE+CUP-GG+NR-NMF+OU provides an SNR of $4.67,8.02,10.21,12.11,16.22,21.22$ and for $\mathrm{SE}+\mathrm{CUP}-\mathrm{GG}+\mathrm{NMF}$ approach is 4.62, 7.22, 9.87, 11.56, 15.07, 19.78. The NR-NMF approach provides an SNR of $1.23,4.96,5.21,9.58,15.86$ and 18.94 .

Table 10. Comparison of Average STOI, \% STOI performance measures for the 30 speech signals corrupted by Pink noise under Input SNRs of -10 $\mathrm{dB},-5 \mathrm{~dB}, 0 \mathrm{~dB}, 5 \mathrm{~dB}, 10 \mathrm{~dB}, 15 \mathrm{~dB}$

\begin{tabular}{|c|c|c|c|c|c|c|c|}
\hline $\begin{array}{l}\text { Input SNR in dB } \\
\text { Method }\end{array}$ & $\begin{array}{l}\text { Performance } \\
\text { Measure }\end{array}$ & -10 & -5 & $\mathbf{0}$ & 5 & 10 & 15 \\
\hline Without Processing & STOI & 0.3245 & 0.5343 & 0.6142 & 0.7543 & 0.8123 & 0.9121 \\
\hline \multirow{2}{*}{$\begin{array}{c}\text { SE+CUP-GG+NR- } \\
\text { NMF+OU }\end{array}$} & $\%$ STOI & 7.09 & 6.23 & 3.68 & 2.45 & 1.78 & 0.89 \\
\hline & STOI & 0.3475 & 0.5676 & 0.6368 & 0.7728 & 0.8267 & 0.9202 \\
\hline \multirow{2}{*}{$\begin{array}{c}\text { SE+CUP-GG+WR- } \\
\text { NMF+OU }\end{array}$} & $\%$ STOI & 7.02 & 6.11 & 3.59 & 2.26 & 1.72 & 0.84 \\
\hline & STOI & 0.3473 & 0.5669 & 0.6362 & 0.7713 & 0.8263 & 0.9198 \\
\hline \multirow{2}{*}{$\mathrm{SE}+\mathrm{CUP}-\mathrm{GG}+\beta-\mathrm{NMF}+\mathrm{OU}$} & $\%$ STOI & 6.87 & 5.86 & 3.52 & 2.45 & 1.73 & 0.85 \\
\hline & STOI & 0.3468 & 0.5656 & 0.6358 & 0.7728 & 0.8264 & 0.9199 \\
\hline
\end{tabular}




\begin{tabular}{|c|c|c|c|c|c|c|c|}
\cline { 2 - 8 } SE+CUP GG+NMF[9] & \% STOI & 6.89 & 5.76 & 3.45 & 2.34 & 1.67 & 0.88 \\
\cline { 2 - 8 } & STOI & 0.3469 & 0.5651 & 0.6354 & 0.772 & 0.8259 & 0.9201 \\
\hline \multirow{2}{*}{ NMF+OU[12] } & \% STOI & 4.14 & 3.38 & 2.98 & 1.15 & 0.86 & 0.67 \\
\cline { 2 - 8 } & STOI & 0.3379 & 0.5524 & 0.6325 & 0.763 & 0.8193 & 0.9182 \\
\hline \multirow{2}{*}{ CUP-GG[9] } & \% STOI & 4.98 & 4.69 & 2.61 & 1.95 & 1.46 & 0.58 \\
\cline { 2 - 8 } & STOI & 0.3406 & 0.5594 & 0.6302 & 0.7690 & 0.8242 & 0.9174 \\
\hline \multirow{2}{*}{ CUP-NG[9] } & \% STOI & 4.59 & 3.81 & 3.29 & 1.23 & 1.15 & 0.54 \\
\cline { 2 - 8 } & STOI & 0.3394 & 0.5547 & 0.6344 & 0.7636 & 0.8216 & 0.917 \\
\hline \multirow{2}{*}{ CUP[8] } & \% STOI & 3.61 & 2.98 & 2.79 & 0.9 & 0.44 & 0.34 \\
\cline { 2 - 9 } & STOI & 0.3362 & 0.5502 & 0.6313 & 0.7611 & 0.8159 & 0.9152 \\
\hline \multirow{2}{*}{ WR NMF[14] } & \% STOI & 2.73 & 2.34 & 2.01 & 0.93 & 0.62 & 0.24 \\
\cline { 2 - 8 } & STOI & 0.3334 & 0.547 & 0.6265 & 0.7613 & 0.8174 & 0.9143 \\
\hline \multirow{2}{*}{ NR NMF[14] } & \% STOI & 2.62 & 2.11 & 1.92 & 0.86 & 0.51 & 0.13 \\
\cline { 2 - 8 } & STOI & 0.3331 & 0.5456 & 0.6259 & 0.7608 & 0.8164 & 0.9133 \\
\hline \multirow{2}{*}{ PR NMF[11] } & \% STOI & 1.62 & 1.23 & 1.03 & 0.43 & 0.14 & 0.09 \\
\cline { 2 - 8 } & STOI & 0.3298 & 0.5409 & 0.6205 & 0.7575 & 0.8134 & 0.9129 \\
\hline \multirow{2}{*}{ HRNR-SPU[13] } & \% STOI & -2.24 & -2.02 & -1.39 & -1.72 & -1.28 & -1.21 \\
\cline { 2 - 8 } & STOI & 0.3172 & 0.5235 & 0.6057 & 0.7413 & 0.8019 & 0.9011 \\
\hline \multirow{2}{*}{ HRNR[5] } & \% STOI & -2.72 & -2.26 & -2.09 & -1.28 & -1.36 & -1.39 \\
\cline { 2 - 8 } & STOI & 0.3157 & 0.5222 & 0.6014 & 0.7447 & 0.8012 & 0.8994 \\
\hline
\end{tabular}

Table 11. Comparison of Average SNR, , Seg SNR performance measures for the 30 speech signals corrupted by Pink noise under Input SNRs of -10 $\mathrm{dB},-5 \mathrm{~dB}, 0 \mathrm{~dB}, 5 \mathrm{~dB}, 10 \mathrm{~dB}, 15 \mathrm{~dB}$

\begin{tabular}{|c|c|c|c|c|c|c|c|}
\hline Method $\quad$ Input SNR in dB & $\begin{array}{l}\text { Performance } \\
\text { Measure }\end{array}$ & -10 & -5 & $\mathbf{0}$ & 5 & 10 & 15 \\
\hline \multirow{3}{*}{$\mathrm{SE}+\mathrm{CUP}-\mathrm{GG}+\mathrm{NR}-\mathrm{NMF}+\mathrm{OU}$} & SNR & 4.67 & 8.02 & 10.21 & 12.11 & 16.22 & 21.22 \\
\hline & SDR & 4.23 & 6.72 & 9.38 & 11.67 & 15.35 & 18.92 \\
\hline & Seg SNR & 3.89 & 6.54 & 7.87 & 11.24 & 15.02 & 17.28 \\
\hline \multirow{3}{*}{$\mathrm{SE}+\mathrm{CUP}-\mathrm{GG}+\mathrm{WR}-\mathrm{NMF}+\mathrm{OU}$} & SNR & 4.28 & 7.8 & 10.86 & 11.84 & 16.84 & 20.84 \\
\hline & SDR & 3.86 & 6.91 & 9.58 & 11.64 & 15.76 & 19.27 \\
\hline & Seg SNR & 3.68 & 6.14 & 7.93 & 10.42 & 15.11 & 16.97 \\
\hline \multirow{3}{*}{$\mathrm{SE}+\mathrm{CUP}-\mathrm{GG}+\boldsymbol{\beta}-\mathrm{NMF}+\mathrm{OU}$} & SNR & 4.69 & 7.26 & 10.44 & 11.51 & 15.84 & 20.21 \\
\hline & SDR & 4.12 & 6.62 & 9.18 & 11.01 & 14.95 & 18.56 \\
\hline & Seg SNR & 3.78 & 5.95 & 7.87 & 10.23 & 14.84 & 16.46 \\
\hline \multirow{3}{*}{ SE+CUP GG+NMF[9] } & SNR & 4.62 & 7.22 & 9.87 & 11.56 & 15.07 & 19.78 \\
\hline & SDR & 3.95 & 6.24 & 8.96 & 10.68 & 14.69 & 17.05 \\
\hline & Seg SNR & 3.69 & 5.87 & 7.57 & 9.89 & 13.68 & 15.87 \\
\hline \multirow{3}{*}{$\mathrm{NMF}+\mathrm{OU}[12]$} & SNR & 3.21 & 4.03 & 7.66 & 11.25 & 15.64 & 17.86 \\
\hline & SDR & 2.25 & 3.11 & 8.69 & 9.96 & 13.96 & 15.76 \\
\hline & Seg SNR & 1.78 & 2.42 & 6.95 & 8.79 & 12.59 & 14.83 \\
\hline \multirow{3}{*}{ CUP-GG[9] } & SNR & 4.46 & 6.98 & 9.06 & 10.96 & 15.82 & 19.32 \\
\hline & SDR & 4.21 & 6.81 & 8.96 & 10.88 & 14.08 & 16.21 \\
\hline & Seg SNR & 3.45 & 5.56 & 7.15 & 9.12 & 12.75 & 14.98 \\
\hline \multirow{3}{*}{ CUP-NG[9] } & SNR & 4.32 & 5.94 & 8.69 & 10.42 & 14.25 & 18.23 \\
\hline & SDR & 3.12 & 3.89 & 7.8 & 9.28 & 13.16 & 15.84 \\
\hline & Seg SNR & 2.26 & 4.76 & 6.87 & 8.67 & 12.12 & 14.25 \\
\hline \multirow{3}{*}{ CUP[8] } & SNR & 2.41 & 3.98 & 7.98 & 9.54 & 13.58 & 17.23 \\
\hline & SDR & 2.87 & 3.21 & 7.12 & 8.78 & 12.84 & 14.95 \\
\hline & Seg SNR & 1.79 & 2.34 & 6.11 & 8.47 & 11.13 & 13.98 \\
\hline \multirow{3}{*}{ NR-NMF[14] } & SNR & 1.23 & 4.96 & 5.21 & 9.58 & 15.86 & 18.94 \\
\hline & SDR & 0.96 & 2.96 & 4.94 & 10.68 & 14.69 & 18.36 \\
\hline & Seg SNR & -3.12 & -0.89 & 3.23 & 6.78 & 10.94 & 13.51 \\
\hline \multirow{3}{*}{ WR-NMF[14] } & SNR & 0.65 & 3.18 & 4.69 & 9.59 & 15.15 & 18.45 \\
\hline & SDR & 0.21 & 2.12 & 4.11 & 9.36 & 13.98 & 17.96 \\
\hline & Seg SNR & -3.67 & -1.42 & 2.45 & 5.04 & 10.87 & 13.26 \\
\hline \multirow{3}{*}{ PR NMF [11] } & SNR & 0.12 & 1.96 & 4.54 & 8.94 & 13.03 & 17.11 \\
\hline & SDR & -2.14 & 1.21 & 3.25 & 7.36 & 12.56 & 15.87 \\
\hline & Seg SNR & -4.56 & -2.34 & 1.34 & 4.21 & 10.34 & 12.23 \\
\hline \multirow{3}{*}{ HRNR-SPU [13] } & SNR & 1.96 & 1.78 & 4.27 & 8.85 & 14.23 & 18.94 \\
\hline & SDR & 1.17 & 1.57 & 2.54 & 5.98 & 11.22 & 14.34 \\
\hline & Seg SNR & 0.28 & 1.24 & 2.08 & 3.03 & 7.32 & 10.23 \\
\hline \multirow{3}{*}{ HRNR[5] } & SNR & 1.18 & 1.67 & 2.54 & 6.81 & 13.14 & 16.71 \\
\hline & SDR & 0.86 & 1.48 & 2.36 & 5.53 & 10.41 & 15.36 \\
\hline & Seg SNR & 0.11 & 0.98 & 1.72 & 2.35 & 6.21 & 9.45 \\
\hline
\end{tabular}


Table 12. Comparison of Average PESQ, $\triangle$ PESQ performance measures for the 30 speech signals corrupted by Modulated Pink noise under Input SNRs of $-10 \mathrm{~dB},-5 \mathrm{~dB}, 0 \mathrm{~dB}, 5 \mathrm{~dB}, 10 \mathrm{~dB}, 15 \mathrm{~dB}$

\begin{tabular}{|c|c|c|c|c|c|c|c|}
\hline Method $\quad$ Input SNR in dB & $\begin{array}{l}\text { Performance } \\
\text { Measure }\end{array}$ & -10 & -5 & $\mathbf{0}$ & 5 & 10 & 15 \\
\hline Without Processing & PESQ & 1.02 & 1.37 & 1.86 & 2.12 & 2.11 & 2.37 \\
\hline \multirow{2}{*}{$\mathrm{SE}+\mathrm{CUP}-\mathrm{GG}+\mathrm{NR}-\mathrm{NMF}+\mathrm{OU}$} & PESQ & 1.31 & 2.12 & 2.78 & 3.03 & 3 & 3.21 \\
\hline & $\triangle$ PESQ & 0.29 & 0.75 & 0.92 & 0.91 & 0.89 & 0.84 \\
\hline \multirow{2}{*}{$\mathrm{SE}+\mathrm{CUP}-\mathrm{GG}+\mathrm{WR}-\mathrm{NMF}+\mathrm{OU}$} & PESQ & 1.3 & 2.08 & 2.75 & 2.98 & 2.93 & 3.14 \\
\hline & $\triangle$ PESQ & 0.28 & 0.71 & 0.89 & 0.86 & 0.82 & 0.77 \\
\hline \multirow{2}{*}{$\mathrm{SE}+\mathrm{CUP}-\mathrm{GG}+\boldsymbol{\beta}-\mathrm{NMF}+\mathrm{OU}$} & PESQ & 1.29 & 2.05 & 2.71 & 2.95 & 2.9 & 3.12 \\
\hline & $\triangle$ PESQ & 0.27 & 0.68 & 0.85 & 0.83 & 0.79 & 0.75 \\
\hline \multirow{2}{*}{ SE+CUP GG+NMF[9] } & PESQ & 1.3 & 2.05 & 2.69 & 2.93 & 2.95 & 3.1 \\
\hline & $\triangle$ PESQ & 0.28 & 0.68 & 0.83 & 0.81 & 0.84 & 0.73 \\
\hline \multirow{2}{*}{$\mathrm{NMF+OU[12]}$} & PESQ & 1.24 & 1.96 & 2.64 & 3.01 & 2.9 & 3.08 \\
\hline & $\triangle$ PESQ & 0.22 & 0.59 & 0.78 & 0.89 & 0.79 & 0.71 \\
\hline \multirow{2}{*}{ CUP-GG[9] } & PESQ & 1.33 & 1.92 & 2.78 & 2.94 & 2.88 & 3.03 \\
\hline & $\triangle$ PESQ & 0.31 & 0.55 & 0.92 & 0.82 & 0.77 & 0.66 \\
\hline \multirow{2}{*}{ CUP-NG[9] } & PESQ & 1.25 & 1.84 & 2.73 & 2.91 & 2.84 & 3.01 \\
\hline & $\triangle$ PESQ & 0.23 & 0.47 & 0.87 & 0.79 & 0.73 & 0.64 \\
\hline \multirow{2}{*}{ CUP[8] } & PESQ & 1.22 & 1.82 & 2.62 & 2.81 & 2.67 & 2.94 \\
\hline & $\triangle$ PESQ & 0.2 & 0.45 & 0.76 & 0.69 & 0.56 & 0.57 \\
\hline \multirow{2}{*}{ WR NMF[14] } & PESQ & 1.26 & 1.8 & 2.58 & 2.87 & 2.9 & 3.09 \\
\hline & $\triangle$ PESQ & 0.24 & 0.43 & 0.72 & 0.75 & 0.79 & 0.72 \\
\hline \multirow{2}{*}{ NR NMF[14] } & PESQ & 2.32 & 3.42 & 4.55 & 5.05 & 5.06 & 5.47 \\
\hline & $\triangle$ PESQ & 0.22 & 0.39 & 0.62 & 0.65 & 0.68 & 0.59 \\
\hline \multirow{2}{*}{ PR NMF[11] } & PESQ & 1.19 & 1.71 & 2.45 & 2.68 & 2.65 & 2.85 \\
\hline & $\triangle$ PESQ & 0.17 & 0.34 & 0.59 & 0.56 & 0.54 & 0.48 \\
\hline \multirow{2}{*}{ HRNR-SPU[13] } & PESQ & 1.16 & 1.66 & 2.27 & 2.57 & 2.53 & 2.76 \\
\hline & $\triangle$ PESQ & 0.14 & 0.29 & 0.41 & 0.45 & 0.42 & 0.39 \\
\hline \multirow{2}{*}{ HRNR[5] } & PESQ & 1.12 & 1.59 & 2.23 & 2.53 & 2.49 & 2.73 \\
\hline & $\triangle$ PESQ & 0.1 & 0.22 & 0.37 & 0.41 & 0.38 & 0.36 \\
\hline
\end{tabular}

In table 12, the Comparison of Average PESQ, $\triangle \mathrm{PESQ}$ performance measures for the 30 speech signals corrupted by Modulated Pink noise under input SNRs of -10 dB, $5 \mathrm{~dB}, 0 \mathrm{~dB}, 5 \mathrm{~dB}, 10 \mathrm{~dB}, 15 \mathrm{~dB}$ is listed. It is observed that the, SE + CUP-GG + NR-NMF + OU provides improved performance than to all compared methods and it provides a $\triangle \mathrm{PESQ}$ value of 0.91 for the input signal corrupted under Modulated Pink noise at OdB SNR. It is observed that SE+CUP-GG + NMF approach provides an PESQ improvement of 0.83. Also it is observed that CUP-GG estimators provides an improved $\triangle$ PESQ value of 0.92 compared to posterior WR-NMF of 0.72 at $0 \mathrm{~dB}$ input SNR. It is noted that the Bayesian estimators shown comparable improvement under low SNRs than to traditional NMF approach. Also it is noted that combination of Bayesian estimator with NMF approach provides improved results in case of Modulated Pink noise.

In table 13 , the Comparison of Average STOI, \%STOI performance measures for the 30 speech signals corrupted by Modulated Pink noise under input SNRs of $-10 \mathrm{~dB}$, $5 \mathrm{~dB}, 0 \mathrm{~dB}, 5 \mathrm{~dB}, 10 \mathrm{~dB}, 15 \mathrm{~dB}$ is listed.It is observed that $\%$ STOI improvement of STOI values for SE + CUP - GG + NR-NMF +OU approach is 6.89, 5.12, 3.34, 2.16, 1.76, 0.89 and for CUP-GG estimator is $4.91,3.95,3.64,1.96$, $0.44,0.27$ and for WR-NMF approach is $2.67,2.12,1.98$, $0.87,0.45,0.3$ at input SNRs of $-10 \mathrm{~dB},-5 \mathrm{~dB}, 0 \mathrm{~dB}, 5 \mathrm{~dB}$, $10 \mathrm{~dB}, 15 \mathrm{~dB}$ respectively. It is noted that at $-10 \mathrm{~d},-5 \mathrm{~dB}$ SNRs there is significant improvement in SE+CUP$\mathrm{GG}+\mathrm{NMF}$ based approaches than to all other methods.

In table 14, the Comparison of Average SNR, SDR, Seg SNR performance measures for the 30 speech signals corrupted by White noise under Input SNRs of $-10 \mathrm{~dB}$, $5 \mathrm{~dB}, 0 \mathrm{~dB}, 5 \mathrm{~dB}, 10 \mathrm{~dB}, 15 \mathrm{~dB}$ is listed. The proposed method SE+CUP-GG+NR-NMF+OU provides an SNR of $4.52,7.96,10.15,12.01,16.24,20.98$ and for SE+CUP-GG+NMF approach is 4.54, 7.12, 9.65, 11.69, 15.98, 19.95. The NR-NMF approach provides an SNR of $1.22,4.72,5.35,9.62,15.89,18.86$.

Table 13. Comparison of Average STOI, \% STOI performance measures for the 30 speech signals corrupted by Modulated Pink noise under Input SNRs of $-10 \mathrm{~dB},-5 \mathrm{~dB}, 0 \mathrm{~dB}, 5 \mathrm{~dB}, 10 \mathrm{~dB}, 15 \mathrm{~dB}$

\begin{tabular}{|c|c|c|c|c|c|c|c|}
\hline $\begin{array}{l}\text { Input SNR in dB } \\
\text { Method }\end{array}$ & $\begin{array}{l}\text { Performance } \\
\text { Measure }\end{array}$ & -10 & -5 & $\mathbf{0}$ & 5 & 10 & 15 \\
\hline Without Processing & STOI & 0.2912 & 0.5042 & 0.5987 & 0.7128 & 0.7965 & 0.8456 \\
\hline \multirow{2}{*}{ SE+CUP-GG+NR-NMF+OU } & $\%$ STOI & 6.89 & 5.12 & 3.34 & 2.16 & 1.76 & 0.89 \\
\hline & STOI & 0.3113 & 0.5300 & 0.6187 & 0.7282 & 0.8105 & 0.8531 \\
\hline \multirow{2}{*}{ SE+CUP-GG+WR-NMF+OU } & $\%$ STOI & 6.65 & 5.11 & 3.16 & 2.13 & 1.71 & 0.74 \\
\hline & STOI & 0.3106 & 0.5299 & 0.6176 & 0.728 & 0.8101 & 0.8519 \\
\hline \multirow{2}{*}{$\mathrm{SE}+\mathrm{CUP}-\mathrm{GG}+\boldsymbol{\beta}-\mathrm{NMF}+\mathrm{OU}$} & $\%$ STOI & 6.61 & 5.28 & 3.19 & 2.11 & 1.68 & 0.69 \\
\hline & STOI & 0.3104 & 0.5308 & 0.6178 & 0.7278 & 0.8099 & 0.8514 \\
\hline
\end{tabular}




\begin{tabular}{|c|c|c|c|c|c|c|c|}
\multirow{4}{*}{ SE+CUP GG+NMF[9] } & \% STOI & 6.57 & 5.32 & 3.21 & 2.02 & 1.63 & 0.67 \\
\cline { 2 - 8 } & STOI & 0.3103 & 0.5310 & 0.6179 & 0.7272 & 0.8095 & 0.8513 \\
\hline \multirow{2}{*}{ NMF+OU[12] } & \% STOI & 4.11 & 3.16 & 2.76 & 1.02 & 0.73 & 0.54 \\
\cline { 2 - 8 } & STOI & 0.3032 & 0.5201 & 0.6152 & 0.7201 & 0.8023 & 0.8502 \\
\hline \multirow{2}{*}{ CUP-GG[9] } & \% STOI & 4.91 & 3.95 & 3.64 & 1.96 & 0.44 & 0.27 \\
\cline { 2 - 8 } & STOI & 0.3055 & 0.5241 & 0.6205 & 0.7268 & 0.8 & 0.8479 \\
\hline \multirow{2}{*}{ CUP-NG[9] } & \% STOI & 4.73 & 3.64 & 2.98 & 1.61 & 0.49 & 0.32 \\
\cline { 2 - 8 } & STOI & 0.305 & 0.5225 & 0.6165 & 0.7243 & 0.8004 & 0.8483 \\
\hline \multirow{2}{*}{ CUP[8] } & \% STOI & 3.51 & 2.76 & 2.49 & 0.95 & 0.26 & 0.12 \\
\cline { 2 - 8 } & STOI & 0.3014 & 0.5181 & 0.6136 & 0.7196 & 0.7986 & 0.8466 \\
\hline \multirow{2}{*}{ WR NMF[14] } & \% STOI & 2.67 & 2.12 & 1.98 & 0.87 & 0.45 & 0.3 \\
\cline { 2 - 8 } & STOI & 0.299 & 0.5149 & 0.6105 & 0.719 & 0.8001 & 0.8481 \\
\hline \multirow{2}{*}{ NR NMF[14] } & \% STOI & 2.45 & 2.01 & 1.78 & 0.74 & 0.49 & 0.11 \\
\cline { 2 - 8 } & STOI & 0.2984 & 0.5143 & 0.6094 & 0.7181 & 0.8004 & 0.8465 \\
\hline \multirow{2}{*}{ PR NMF[11] } & \% STOI & 1.56 & 1.17 & 0.98 & 0.26 & 0.11 & 0.1 \\
\cline { 2 - 8 } & STOI & 0.2957 & 0.5101 & 0.6046 & 0.7146 & 0.7974 & 0.8465 \\
\hline \multirow{2}{*}{ HRNR-SPU[13] } & \% STOI & -2.54 & -2.29 & -1.53 & -1.85 & -1.39 & -1.36 \\
\cline { 2 - 8 } & STOI & 0.2838 & 0.4926 & 0.5895 & 0.6996 & 0.7854 & 0.8341 \\
\hline \multirow{2}{*}{ HRNR[5] } & \% STOI & -2.97 & -2.43 & -2.18 & -1.39 & -1.54 & -1.76 \\
\cline { 2 - 8 } & STOI & 0.2828 & 0.4922 & 0.5858 & 0.7031 & 0.7844 & 0.8309 \\
\hline
\end{tabular}

Table 14. Comparison of Average SNR, SDR, Segmental SNR performance measures for the 30 speech signals corrupted by Modulated Pink noise under Input SNRs of $-10 \mathrm{~dB},-5 \mathrm{~dB}, 0 \mathrm{~dB}, 5 \mathrm{~dB}, 10 \mathrm{~dB}, 15 \mathrm{~dB}$

\begin{tabular}{|c|c|c|c|c|c|c|c|}
\hline $\begin{array}{l}\text { Input SNR in dB } \\
\text { Method }\end{array}$ & Performance Measure & -10 & -5 & $\mathbf{0}$ & 5 & 10 & 15 \\
\hline \multirow{3}{*}{$\mathrm{SE}+\mathrm{CUP}-\mathrm{GG}+\mathrm{NR}-\mathrm{NMF}+\mathrm{OU}$} & SNR & 4.52 & 7.96 & 10.15 & 12.01 & 16.24 & 20.98 \\
\hline & SDR & 4.11 & 6.58 & 9.21 & 11.52 & 15.12 & 18.79 \\
\hline & Seg SNR & 3.76 & 6.42 & 7.65 & 11.12 & 14.94 & 17.58 \\
\hline \multirow{3}{*}{$\mathrm{SE}+\mathrm{CUP}-\mathrm{GG}+\mathrm{WR}-\mathrm{NMF}+\mathrm{OU}$} & SNR & 4.05 & 7.64 & 10.68 & 11.78 & 16.95 & 20.64 \\
\hline & SDR & 3.79 & 6.78 & 9.51 & 11.75 & 15.89 & 19.46 \\
\hline & Seg SNR & 3.62 & 6.02 & 7.85 & 10.29 & 14.95 & 16.86 \\
\hline \multirow{3}{*}{$\mathrm{SE}+\mathrm{CUP}-\mathrm{GG}+\beta-\mathrm{NMF}+\mathrm{OU}$} & SNR & 4.52 & 7.03 & 10.57 & 11.62 & 15.78 & 20.06 \\
\hline & SDR & 4.03 & 6.51 & 9.08 & 10.86 & 14.99 & 18.68 \\
\hline & Seg SNR & 3.71 & 5.84 & 7.81 & 10.95 & 14.78 & 16.24 \\
\hline \multirow{3}{*}{ SE+CUP GG+NMF[9] } & SNR & 4.54 & 7.12 & 9.65 & 11.69 & 15.98 & 19.95 \\
\hline & SDR & 3.84 & 6.72 & 8.82 & 10.54 & 14.52 & 17.24 \\
\hline & Seg SNR & 3.46 & 5.84 & 7.63 & 9.97 & 13.95 & 15.75 \\
\hline \multirow{3}{*}{$\mathrm{NMF}+\mathrm{OU}[12]$} & SNR & 3.12 & 4.21 & 7.81 & 11.21 & 15.42 & 17.89 \\
\hline & SDR & 2.28 & 3.09 & 8.76 & 9.21 & 13.74 & 15.64 \\
\hline & Seg SNR & 1.68 & 2.31 & 6.86 & 8.54 & 12.34 & 14.68 \\
\hline \multirow{3}{*}{ CUP-GG[9] } & SNR & 4.63 & 6.89 & 9.21 & 10.93 & 15.72 & 19.23 \\
\hline & SDR & 4.14 & 6.65 & 8.78 & 10.95 & 14.26 & 16.35 \\
\hline & Seg SNR & 3.12 & 5.23 & 7.02 & 9.02 & 12.23 & 14.76 \\
\hline \multirow{3}{*}{ CUP-NG[9] } & SNR & 4.13 & 5.78 & 8.51 & 10.25 & 14.11 & 18.14 \\
\hline & SDR & 3.01 & 3.81 & 7.64 & 9.32 & 13.28 & 15.89 \\
\hline & Seg SNR & 2.13 & 4.35 & 6.68 & 8.52 & 11.92 & 14.11 \\
\hline \multirow{3}{*}{ CUP[8] } & SNR & 3.06 & 3.76 & 7.75 & 9.21 & 13.14 & 17.21 \\
\hline & SDR & 2.81 & 3.23 & 7.28 & 8.64 & 12.56 & 15.24 \\
\hline & Seg SNR & 1.68 & 2.56 & 5.98 & 8.21 & 10.89 & 13.69 \\
\hline \multirow{3}{*}{ NR-NMF[14] } & SNR & 1.22 & 4.72 & 5.35 & 9.62 & 15.89 & 18.86 \\
\hline & SDR & 0.99 & 2.99 & 4.85 & 10.85 & 14.86 & 18.51 \\
\hline & Seg SNR & -3.25 & -0.92 & 3.12 & 6.64 & 10.85 & 13.44 \\
\hline \multirow{3}{*}{ WR-NMF[14] } & SNR & 0.58 & 3.12 & 4.58 & 9.45 & 15.01 & 18.65 \\
\hline & SDR & 0.19 & 2.52 & 4.21 & 9.24 & 14.05 & 18.11 \\
\hline & Seg SNR & -3.78 & -1.49 & 2.61 & 5.14 & 10.68 & 13.56 \\
\hline \multirow{3}{*}{ PR NMF [11] } & SNR & 0.11 & 2.01 & 4.35 & 8.85 & 12.85 & 16.89 \\
\hline & SDR & -2.26 & 1.18 & 3.29 & 7.15 & 12.34 & 15.89 \\
\hline & Seg SNR & -4.67 & -2.47 & 1.41 & 4.02 & 10.42 & 12.54 \\
\hline \multirow{3}{*}{ HRNR-SPU [13] } & SNR & -5.34 & -1.31 & 2.56 & 7.28 & 12.25 & 16.67 \\
\hline & SDR & -6.65 & -2.14 & 1.22 & 5.85 & 10.98 & 15.86 \\
\hline & Seg SNR & -8.91 & -3.54 & -1.24 & 2.95 & 7.12 & 10.02 \\
\hline \multirow{3}{*}{ HRNR[5] } & SNR & -5.86 & -1.35 & 0.51 & 6.57 & 10.42 & 15.11 \\
\hline & SDR & -7.89 & -2.89 & -0.31 & 5.48 & 10.25 & 15.17 \\
\hline & Seg SNR & -9.99 & -4.35 & -1.31 & 2.12 & 5.98 & 9.36 \\
\hline
\end{tabular}


Table 15. Comparison of Average PESQ, $\triangle$ PESQ performance measures for the 30 speech signals corrupted by Factory noise under Input SNRs of $10 \mathrm{~dB},-5 \mathrm{~dB}, 0 \mathrm{~dB}, 5 \mathrm{~dB}, 10 \mathrm{~dB}, 15 \mathrm{~dB}$

\begin{tabular}{|c|c|c|c|c|c|c|c|}
\hline $\begin{array}{ll}\text { Input SNR in dB } \\
\text { Method }\end{array}$ & $\begin{array}{l}\text { Performance } \\
\text { Measure }\end{array}$ & -10 & -5 & $\mathbf{0}$ & 5 & 10 & 15 \\
\hline Without Processing & PESQ & 1.28 & 1.53 & 1.93 & 2.07 & 2.35 & 2.67 \\
\hline \multirow{2}{*}{$\mathrm{SE}+\mathrm{CUP}-\mathrm{GG}+\mathrm{NR}-\mathrm{NMF}+\mathrm{OU}$} & PESQ & 1.55 & 2.2 & 2.91 & 3.04 & 3.26 & 3.5 \\
\hline & $\triangle$ PESQ & 0.27 & 0.67 & 0.98 & 0.97 & 0.91 & 0.83 \\
\hline \multirow{2}{*}{ SE+CUP-GG+WR-NMF+OU } & PESQ & 1.53 & 2.12 & 2.85 & 3.02 & 3.24 & 3.51 \\
\hline & $\triangle$ PESQ & 0.25 & 0.59 & 0.92 & 0.95 & 0.89 & 0.84 \\
\hline \multirow{2}{*}{$\mathrm{SE}+\mathrm{CUP}-\mathrm{GG}+\boldsymbol{\beta}-\mathrm{NMF}+\mathrm{OU}$} & PESQ & 1.51 & 2.04 & 2.82 & 2.98 & 3.17 & 3.46 \\
\hline & $\triangle$ PESQ & 0.23 & 0.51 & 0.89 & 0.91 & 0.82 & 0.79 \\
\hline \multirow{2}{*}{ SE+CUP GG+NMF[9] } & PESQ & 1.49 & 1.95 & 2.74 & 2.86 & 3.11 & 3.4 \\
\hline & $\triangle$ PESQ & 0.21 & 0.42 & 0.81 & 0.79 & 0.76 & 0.73 \\
\hline \multirow{2}{*}{$\mathrm{NMF}+\mathrm{OU}[12]$} & PESQ & 1.36 & 1.85 & 2.57 & 2.74 & 3 & 3.27 \\
\hline & $\triangle$ PESQ & 0.08 & 0.32 & 0.64 & 0.67 & 0.65 & 0.6 \\
\hline \multirow{2}{*}{ CUP-GG[9] } & PESQ & 1.42 & 1.92 & 2.65 & 2.74 & 3 & 3.3 \\
\hline & $\triangle$ PESQ & 0.14 & 0.39 & 0.72 & 0.67 & 0.65 & 0.63 \\
\hline \multirow{2}{*}{ CUP-NG[9] } & PESQ & 1.39 & 1.88 & 2.61 & 2.75 & 2.99 & 3.29 \\
\hline & $\triangle$ PESQ & 0.11 & 0.35 & 0.68 & 0.68 & 0.64 & 0.62 \\
\hline \multirow{2}{*}{ CUP[8] } & PESQ & 1.32 & 1.83 & 2.55 & 2.65 & 2.91 & 3.21 \\
\hline & $\triangle$ PESQ & 0.04 & 0.3 & 0.62 & 0.58 & 0.56 & 0.54 \\
\hline \multirow{2}{*}{ WR NMF[14] } & PESQ & 1.4 & 1.9 & 2.55 & 2.75 & 2.99 & 3.26 \\
\hline & $\triangle$ PESQ & 0.12 & 0.37 & 0.62 & 0.68 & 0.64 & 0.59 \\
\hline \multirow{2}{*}{ NR NMF[14] } & PESQ & 1.39 & 1.92 & 2.58 & 2.74 & 3 & 3.24 \\
\hline & $\triangle$ PESQ & 0.11 & 0.39 & 0.65 & 0.67 & 0.65 & 0.57 \\
\hline \multirow{2}{*}{ PR NMF[11] } & PESQ & 1.37 & 1.84 & 2.42 & 2.59 & 2.83 & 3.12 \\
\hline & $\triangle$ PESQ & 0.09 & 0.31 & 0.49 & 0.52 & 0.48 & 0.45 \\
\hline \multirow{2}{*}{ HRNR-SPU[13] } & PESQ & 1.35 & 1.8 & 2.38 & 2.49 & 2.7 & 2.98 \\
\hline & $\triangle$ PESQ & 0.07 & 0.27 & 0.45 & 0.42 & 0.35 & 0.31 \\
\hline \multirow{2}{*}{ HRNR[5] } & PESQ & 1.34 & 1.7 & 2.31 & 2.38 & 2.62 & 2.92 \\
\hline & $\triangle$ PESQ & 0.06 & 0.17 & 0.38 & 0.31 & 0.27 & 0.25 \\
\hline
\end{tabular}

In table 15, the Comparison of Average PESQ, $\triangle \mathrm{PESQ}$ performance measures for the 30 speech signals corrupted by Factory noise under input SNRs of $-10 \mathrm{~dB},-5 \mathrm{~dB}, 0 \mathrm{~dB}$, $5 \mathrm{~dB}, 10 \mathrm{~dB}, 15 \mathrm{~dB}$ is listed. It is observed that the, $\mathrm{SE}+$ CUP-GG + NR-NMF + OU provides improved performance than to all compared methods and it provides a $\triangle$ PESQ value of 0.98 for the input signal corrupted under Factory noise at OdB SNR. It is observed that SE+CUP-GG + NMF approach provides an PESQ improvement of 0.81 . Also it is observed that CUP-GG estimators provides an improved $\triangle \mathrm{PESQ}$ value of 0.72 compared to posterior

WR-NMF of 0.62 at $0 \mathrm{~dB}$ input SNR. It is noted that the Bayesian estimators shown comparable improvement under low SNRs than to traditional NMF approach. Also noted that combination of Bayesian estimator with NMF approach provides improved results in case of Factory noise

In table 16, the Comparison of Average STOI, \%STOI performance measures for the 30 speech signals corrupted by Factory noise under Input SNRs of $-10 \mathrm{~dB},-5 \mathrm{~dB}, 0 \mathrm{~dB}$, $5 \mathrm{~dB}, 10 \mathrm{~dB}, 15 \mathrm{~dB}$ is listed. It is observed that $\%$ STOI improvement of STOI values for

$\mathrm{SE}+\mathrm{CUP}-\mathrm{GG}+\mathrm{NR}-\mathrm{NMF}+\mathrm{OU}$ approach is 6.67 , $5.63,3.54,2.25,1.63,0.73$ and for CUP-GG estimator is $3.98,3.18,2.59,1.79,0.85,0.31$ and for WR-NMF approach is $2.62,2.21,1.86,0.79,0.38,0.12$ at input SNRs of $-10 \mathrm{~dB},-5 \mathrm{~dB}, 0 \mathrm{~dB}, 5 \mathrm{~dB}, 10 \mathrm{~dB}, 15 \mathrm{~dB}$ respectively. It is noted that at $-10 \mathrm{~d},-5 \mathrm{~dB}$ SNRs there is significant improvement in SE+CUP-GG+NMF based approaches than to all other methods.

In Table 17, the Comparison of Average SNR, SDR, Seg SNR performance measures for the 30 speech signals corrupted by Factory noise under Input SNRs of $-10 \mathrm{~dB}$, $5 \mathrm{~dB}, 0 \mathrm{~dB}, 5 \mathrm{~dB}, 10 \mathrm{~dB}, 15 \mathrm{~dB}$ is listed. The proposed method SE+CUP-GG+NR-NMF+OU provides an SNR of $4.41,7.85,10.12,11.97,15.98,20.85$ and for SE+CUP-GG+NMF approach is 4.54, 7.12, 9.65, 11.69, 15.98, 19.95. The NR-NMF approach provides an SNR of $1.02,5.49,5.21,9.51,15.67,15.67$.

Table 16. Comparison of Average STOI, \% STOI performance measures for the 30 speech signals corrupted by Factory noise under Input SNRs of $10 \mathrm{~dB},-5 \mathrm{~dB}, 0 \mathrm{~dB}, 5 \mathrm{~dB}, 10 \mathrm{~dB}, 15 \mathrm{~dB}$

\begin{tabular}{|c|c|c|c|c|c|c|c|}
\hline $\begin{array}{l}\text { Input SNR in dB } \\
\text { Method }\end{array}$ & $\begin{array}{l}\text { Performance } \\
\text { Measure }\end{array}$ & -10 & -5 & $\mathbf{0}$ & 5 & 10 & 15 \\
\hline Without Processing & STOI & 0.3109 & 0.5122 & 0.6011 & 0.7476 & 0.8098 & 0.8976 \\
\hline \multirow{2}{*}{$\mathrm{SE}+\mathrm{CUP}-\mathrm{GG}+\mathrm{NR}-\mathrm{NMF}+\mathrm{OU}$} & \% STOI & 6.67 & 5.63 & 3.54 & 2.25 & 1.63 & 0.73 \\
\hline & STOI & 0.3316 & 0.5411 & 0.6224 & 0.7644 & 0.8221 & 0.9042 \\
\hline \multirow{2}{*}{$\mathrm{SE}+\mathrm{CUP}-\mathrm{GG}+\mathrm{WR}-\mathrm{NMF}+\mathrm{OU}$} & \% STOI & 6.58 & 5.45 & 3.35 & 2.16 & 1.59 & 0.67 \\
\hline & STOI & 0.3313 & 0.5401 & 0.6212 & 0.7638 & 0.8226 & 0.9036 \\
\hline \multirow{2}{*}{$\mathrm{SE}+\mathrm{CUP}-\mathrm{GG}+\boldsymbol{\beta}-\mathrm{NMF}+\mathrm{OU}$} & $\%$ STOI & 6.46 & 5.32 & 3.21 & 2.12 & 1.52 & 0.64 \\
\hline & STOI & 0.3310 & 0.5394 & 0.6204 & 0.7634 & 0.8221 & 0.9033 \\
\hline \multirow{2}{*}{ SE+CUP GG+NMF[9] } & \% STOI & 6.12 & 5.11 & 3.09 & 1.92 & 1.45 & 0.58 \\
\hline & STOI & 0.3299 & 0.5384 & 0.6197 & 0.7619 & 0.8215 & 0.9028 \\
\hline
\end{tabular}




\begin{tabular}{|c|c|c|c|c|c|c|c|}
\cline { 2 - 8 } NMF+OU[12] & \% STOI & 4.09 & 3.22 & 2.53 & 0.93 & 0.62 & 0.45 \\
\cline { 2 - 8 } & STOI & 0.3236 & 0.5287 & 0.6163 & 0.7545 & 0.8148 & 0.9016 \\
\hline \multirow{2}{*}{ CUP-GG[9] } & \% STOI & 3.98 & 3.18 & 2.59 & 1.79 & 0.85 & 0.31 \\
\cline { 2 - 8 } & STOI & 0.3233 & 0.5285 & 0.6167 & 0.7609 & 0.8167 & 0.9004 \\
\hline \multirow{2}{*}{ CUP-NG[9] } & \% STOI & 3.61 & 2.98 & 2.91 & 1.18 & 0.69 & 0.19 \\
\cline { 2 - 8 } & STOI & 0.3221 & 0.5275 & 0.6186 & 0.7564 & 0.8154 & 0.8993 \\
\hline \multirow{2}{*}{ CUP[8] } & \% STOI & 3.02 & 2.56 & 1.98 & 0.69 & 0.22 & 0.07 \\
\cline { 2 - 8 } & STOI & 0.3203 & 0.5253 & 0.613 & 0.7527 & 0.8116 & 0.8982 \\
\hline \multirow{2}{*}{ WR NMF[14] } & \% STOI & 2.62 & 2.21 & 1.86 & 0.79 & 0.38 & 0.12 \\
\cline { 2 - 8 } & STOI & 0.3190 & 0.5235 & 0.6123 & 0.7535 & 0.8128 & 0.8987 \\
\hline \multirow{2}{*}{ NR NMF[14] } & \% STOI & 2.34 & 1.97 & 1.64 & 0.67 & 0.23 & 0.13 \\
\cline { 2 - 8 } & STOI & 0.3181 & 0.5223 & 0.6110 & 0.7526 & 0.8117 & 0.8988 \\
\hline \multirow{2}{*}{ PR NMF[11] } & \% STOI & 1.62 & 1.19 & 0.92 & 0.21 & 0.06 & 0.09 \\
\cline { 2 - 8 } & STOI & 0.3159 & 0.5183 & 0.6066 & 0.7491 & 0.8103 & 0.8984 \\
\hline \multirow{2}{*}{ HRNR-SPU[13] } & \% STOI & -2.39 & -2.12 & -1.45 & -1.72 & -1.31 & -1.29 \\
\cline { 2 - 8 } & STOI & 0.3035 & 0.5013 & 0.5924 & 0.7347 & 0.7992 & 0.886 \\
\hline \multirow{2}{*}{ HRNR[5] } & \% STOI & -2.86 & -2.35 & -2.16 & -1.35 & -1.48 & -1.52 \\
\cline { 2 - 8 } & STOI & 0.3020 & 0.5002 & 0.5881 & 0.7375 & 0.7978 & 0.884 \\
\hline
\end{tabular}

Table 17. Comparison of Average SNR, SDR, Seg SNR performance measures for the 30 speech signals corrupted by Factory noise under Input SNRs of $-10 \mathrm{~dB},-5 \mathrm{~dB}, 0 \mathrm{~dB}, 5 \mathrm{~dB}, 10 \mathrm{~dB}, 15 \mathrm{~dB}$

\begin{tabular}{|c|c|c|c|c|c|c|c|}
\hline Method & $\begin{array}{l}\text { Performance } \\
\text { Measure }\end{array}$ & -10 & -5 & $\mathbf{0}$ & 5 & 10 & 15 \\
\hline \multirow{3}{*}{$\mathrm{SE}+\mathrm{CUP}-\mathrm{GG}+\mathrm{NR}-\mathrm{NMF}+\mathrm{OU}$} & SNR & 4.41 & 7.85 & 10.12 & 11.97 & 15.98 & 20.85 \\
\hline & SDR & 4.02 & 6.41 & 9.11 & 11.36 & 15.18 & 18.78 \\
\hline & Seg SNR & 3.71 & 6.32 & 7.48 & 10.98 & 14.78 & 17.36 \\
\hline \multirow{3}{*}{ SE+CUP-GG+WR-NMF+OU } & SNR & 3.98 & 7.58 & 10.57 & 11.69 & 16.84 & 20.25 \\
\hline & SDR & 3.58 & 6.65 & 9.47 & 11.67 & 15.76 & 19.25 \\
\hline & Seg SNR & 3.54 & 5.97 & 7.76 & 10.25 & 14.84 & 16.73 \\
\hline \multirow{3}{*}{$\mathrm{SE}+\mathrm{CUP}-\mathrm{GG}+\boldsymbol{\beta}-\mathrm{NMF}+\mathrm{OU}$} & SNR & 4.46 & 6.97 & 10.46 & 11.57 & 15.62 & 19.95 \\
\hline & SDR & 3.98 & 6.43 & 8.96 & 10.98 & 14.82 & 18.45 \\
\hline & Seg SNR & 3.68 & 5.72 & 7.65 & 10.86 & 14.87 & 16.13 \\
\hline \multirow{3}{*}{ SE+CUP GG+NMF[9] } & SNR & 4.35 & 6.97 & 9.54 & 11.57 & 15.21 & 19.78 \\
\hline & SDR & 3.62 & 6.59 & 8.67 & 10.42 & 14.47 & 16.75 \\
\hline & Seg SNR & 3.25 & 5.75 & 7.54 & 9.74 & 13.78 & 15.48 \\
\hline \multirow{3}{*}{$\mathrm{NMF}+\mathrm{OU}[12]$} & SNR & 3.04 & 4.12 & 8.57 & 11.26 & 15.27 & 17.96 \\
\hline & SDR & 2.31 & 3.26 & 8.54 & 9.54 & 13.98 & 15.84 \\
\hline & Seg SNR & 1.57 & 2.29 & 6.57 & 8.25 & 12.11 & 14.54 \\
\hline \multirow{3}{*}{ CUP-GG[9] } & SNR & 4.42 & 6.58 & 8.97 & 10.86 & 14.98 & 19.1 \\
\hline & SDR & 3.89 & 6.25 & 8.59 & 10.41 & 13.87 & 16.14 \\
\hline & Seg SNR & 3.04 & 5.03 & 6.98 & 8.87 & 12.03 & 14.58 \\
\hline \multirow{3}{*}{ CUP-NG[9] } & SNR & 3.96 & 5.67 & 8.34 & 10.11 & 13.95 & 17.86 \\
\hline & SDR & 2.94 & 3.65 & 7.58 & 9.14 & 13.11 & 15.75 \\
\hline & Seg SNR & 1.96 & 4.12 & 6.46 & 8.34 & 11.78 & 14.03 \\
\hline \multirow{3}{*}{ CUP[8] } & SNR & 3.21 & 3.78 & 7.65 & 9.11 & 13.04 & 16.98 \\
\hline & SDR & 2.68 & 3.12 & 7.02 & 8.52 & 12.38 & 15.01 \\
\hline & Seg SNR & 1.57 & 2.38 & 5.74 & 8.01 & 10.65 & 13.48 \\
\hline \multirow{3}{*}{ NR-NMF[14] } & SNR & 1.02 & 4.59 & 5.21 & 9.51 & 15.67 & 18.67 \\
\hline & SDR & 0.78 & 2.83 & 4.67 & 10.68 & 14.69 & 18.34 \\
\hline & Seg SNR & -3.12 & -0.95 & 3.08 & 6.45 & 10.59 & 13.22 \\
\hline \multirow{3}{*}{ WR-NMF[14] } & SNR & 0.46 & 2.98 & 4.35 & 9.21 & 14.97 & 18.54 \\
\hline & SDR & 0.17 & 2.41 & 4.1 & 8.95 & 13.86 & 17.99 \\
\hline & Seg SNR & -3.86 & -1.58 & 2.83 & 5.11 & 10.45 & 13.14 \\
\hline \multirow{3}{*}{ PR NMF [11] } & SNR & 0.08 & 1.89 & 4.112 & 8.67 & 12.69 & 16.68 \\
\hline & SDR & -2.35 & 1.26 & 3.12 & 6.98 & 11.96 & 15.24 \\
\hline & Seg SNR & -4.85 & -2.56 & 1.57 & 3.98 & 10.21 & 12.48 \\
\hline \multirow{3}{*}{ HRNR-SPU [13] } & SNR & -5.42 & -1.42 & 2.43 & 7.12 & 12.03 & 16.54 \\
\hline & SDR & -6.76 & -2.25 & 1.34 & 5.69 & 10.84 & 15.75 \\
\hline & Seg SNR & -8.76 & -3.38 & -1.11 & 2.87 & 6.95 & 9.96 \\
\hline \multirow{3}{*}{ HRNR[5] } & SNR & -5.74 & -1.32 & 0.48 & 5.34 & 10.51 & 15.16 \\
\hline & SDR & -7.92 & -2.95 & -0.38 & 5.45 & 10.12 & 15.02 \\
\hline & Seg SNR & -9.98 & -4.42 & -1.39 & 2.18 & 5.87 & 9.25 \\
\hline
\end{tabular}


Table 18. Comparison of Average PESQ, $\triangle \mathrm{PESQ}$ performance measures for the 30 speech signals corrupted by Car noise under Input SNRs of -10 dB, $-5 \mathrm{~dB}, 0 \mathrm{~dB}, 5 \mathrm{~dB}, 10 \mathrm{~dB}, 15 \mathrm{~dB}$.

\begin{tabular}{|c|c|c|c|c|c|c|c|}
\hline $\begin{array}{l}\text { Input SNR in dB } \\
\text { Method }\end{array}$ & $\begin{array}{l}\text { Performance } \\
\text { Measure }\end{array}$ & -10 & -5 & $\mathbf{0}$ & 5 & 10 & 15 \\
\hline Without Processing & PESQ & 1.12 & 1.63 & 1.87 & 2.12 & 2.23 & 2.57 \\
\hline \multirow{2}{*}{$\mathrm{SE}+\mathrm{CUP}-\mathrm{GG}+\mathrm{NR}-\mathrm{NMF}+\mathrm{OU}$} & PESQ & 1.35 & 2.25 & 2.76 & 3.05 & 3.08 & 3.31 \\
\hline & $\triangle$ PESQ & 0.23 & 0.62 & 0.89 & 0.93 & 0.85 & 0.74 \\
\hline \multirow{2}{*}{ SE+CUP-GG+WR-NMF+OU } & PESQ & 1.37 & 2.22 & 2.78 & 3.04 & 3.1 & 3.38 \\
\hline & $\triangle$ PESQ & 0.25 & 0.59 & 0.91 & 0.92 & 0.87 & 0.81 \\
\hline \multirow{2}{*}{$\mathrm{SE}+\mathrm{CUP}-\mathrm{GG}+\boldsymbol{\beta}-\mathrm{NMF}+\mathrm{OU}$} & PESQ & 1.35 & 2.19 & 2.73 & 3.01 & 3.07 & 3.35 \\
\hline & $\triangle$ PESQ & 0.23 & 0.56 & 0.86 & 0.89 & 0.84 & 0.78 \\
\hline \multirow{2}{*}{ SE+CUP GG+NMF[9] } & PESQ & 1.33 & 2.09 & 2.66 & 2.94 & 2.99 & 3.29 \\
\hline & $\triangle$ PESQ & 0.21 & 0.46 & 0.79 & 0.82 & 0.76 & 0.72 \\
\hline \multirow{2}{*}{$\mathrm{NMF}+\mathrm{OU}[12]$} & PESQ & 1.2 & 2 & 2.52 & 2.84 & 2.88 & 3.18 \\
\hline & $\triangle$ PESQ & 0.08 & 0.37 & 0.65 & 0.72 & 0.65 & 0.61 \\
\hline \multirow{2}{*}{ CUP-GG[9] } & PESQ & 1.26 & 2.02 & 2.54 & 2.81 & 2.86 & 3.18 \\
\hline & $\triangle$ PESQ & 0.14 & 0.39 & 0.67 & 0.69 & 0.63 & 0.61 \\
\hline \multirow{2}{*}{ CUP-NG[9] } & PESQ & 1.23 & 1.99 & 2.48 & 2.75 & 2.82 & 3.13 \\
\hline & $\triangle$ PESQ & 0.11 & 0.36 & 0.61 & 0.63 & 0.59 & 0.56 \\
\hline \multirow{2}{*}{ CUP[8] } & PESQ & 1.19 & 1.95 & 2.54 & 2.74 & 2.77 & 3.12 \\
\hline & $\triangle$ PESQ & 0.07 & 0.32 & 0.67 & 0.62 & 0.54 & 0.55 \\
\hline \multirow{2}{*}{ WR NMF[14] } & PESQ & 1.24 & 2.01 & 2.46 & 2.77 & 2.81 & 3.09 \\
\hline & $\triangle$ PESQ & 0.12 & 0.38 & 0.59 & 0.65 & 0.58 & 0.52 \\
\hline \multirow{2}{*}{ NR NMF[14] } & PESQ & 1.21 & 1.99 & 2.46 & 2.79 & 2.84 & 3.14 \\
\hline & $\triangle$ PESQ & 0.09 & 0.36 & 0.59 & 0.67 & 0.61 & 0.57 \\
\hline \multirow{2}{*}{ PR NMF[11] } & PESQ & 1.18 & 1.94 & 2.32 & 2.61 & 2.66 & 2.96 \\
\hline & $\triangle$ PESQ & 0.06 & 0.31 & 0.45 & 0.49 & 0.43 & 0.39 \\
\hline \multirow{2}{*}{ HRNR-SPU[13] } & PESQ & 1.15 & 1.84 & 2.26 & 2.5 & 2.54 & 2.85 \\
\hline & $\triangle$ PESQ & 0.03 & 0.21 & 0.39 & 0.38 & 0.31 & 0.28 \\
\hline \multirow{2}{*}{ HRNR[5] } & PESQ & 1.13 & 1.79 & 2.25 & 2.46 & 2.51 & 2.81 \\
\hline & $\triangle$ PESQ & 0.01 & 0.16 & 0.38 & 0.34 & 0.28 & 0.24 \\
\hline
\end{tabular}

In table 18, the Comparison of Average PESQ, $\triangle \mathrm{PESQ}$ performance measures for the 30 speech signals corrupted by Car noise under input SNRs of $-10 \mathrm{~dB},-5 \mathrm{~dB}, 0 \mathrm{~dB}, 5$ $\mathrm{dB}, 10 \mathrm{~dB}, 15 \mathrm{~dB}$ is listed. It is observed that the, $\mathrm{SE}+$ CUP-GG + NR-NMF + OU provides improved performance than to all compared methods and it provides a $\triangle \mathrm{PESQ}$ value of 0.89 for the input signal corrupted under Car noise at OdB SNR. It is observed that $\mathrm{SE}+\mathrm{CUP}-\mathrm{GG}+\mathrm{NMF}$ approach provides an PESQ improvement of 0.79. Also it is observed that CUP-GG estimators provides an improved $\triangle \mathrm{PESQ}$ value of 0.67 compared to posterior WR-NMF of 0.59 at $0 \mathrm{~dB}$ input SNR. It is noted that the Bayesian estimators shown comparable improvement under low SNRs than to traditional NMF approach. Also noted that combination of Bayesian estimator with NMF approach provides improved results in case of Car noise

In table 19, the Comparison of Average STOI, \%STOI performance measures for the 30 speech signals corrupted by Car noise under Input SNRs of $-10 \mathrm{~dB},-5 \mathrm{~dB}, 0 \mathrm{~dB}, 5$ $\mathrm{dB}, 10 \mathrm{~dB}, 15 \mathrm{~dB}$ is listed. It is observed that \% STOI improvement of STOI values for SE + CUP - GG + NR $\mathrm{NMF}+\mathrm{OU}$ approach is $6.92,5.84,3.76,2.63,1.97,0.95$ and for CUP-GG estimator is 4.97, 3.78, 3.21, 1.78, 0.49, 0.43 and for WR-NMF approach is $2.71,2.18,1.99,0.89$, $0.36,0.14$ at input SNRs of $-10 \mathrm{~dB},-5 \mathrm{~dB}, 0 \mathrm{~dB}, 5 \mathrm{~dB}, 10$ $\mathrm{dB}, 15 \mathrm{~dB}$ respectively. It is noted that at $-10 \mathrm{~dB},-5 \mathrm{~dB}$ SNRs there is significant improvement in SE+CUP$\mathrm{GG}+\mathrm{NMF}$ based approaches than to all other methods.

In Table 20, the Comparison of Average SNR, SDR, Seg SNR performance measures for the 30 speech signals corrupted by Car noise under Input SNRs of $-10 \mathrm{~dB},-5 \mathrm{~dB}$ $0 \mathrm{~dB}, 5 \mathrm{~dB}, 10 \mathrm{~dB}, 15 \mathrm{~dB}$ is listed. The proposed method $\mathrm{SE}+\mathrm{CUP}-\mathrm{GG}+\mathrm{NR}-\mathrm{NMF}+\mathrm{OU}$ provides an SNR of 4.89, $8.14,10.13,12.05,16.17,21.18$ and for SE+CUPGG+NMF approach is 4.43, 7.04, 9.74, 11.47, 14.97, 19.67. The NR-NMF approach provides an SNR of 1.17, $4.85,5.12,9.47,15.76,18.85$.

Table 19. Comparison of Average STOI, \% STOI performance measures for the 30 speech signals corrupted by Car noise under Input SNRs of -10 $\mathrm{dB},-5 \mathrm{~dB}, 0 \mathrm{~dB}, 5 \mathrm{~dB}, 10 \mathrm{~dB}, 15 \mathrm{~dB}$.

\begin{tabular}{|c|c|c|c|c|c|c|c|}
\hline $\begin{array}{l}\text { Input SNR in dB } \\
\text { Method }\end{array}$ & $\begin{array}{c}\text { Performance } \\
\text { Measure }\end{array}$ & -10 & -5 & $\mathbf{0}$ & 5 & 10 & 15 \\
\hline Without Processing & STOI & 0.3122 & 0.5349 & 0.6292 & 0.7682 & 0.8221 & 0.9532 \\
\hline \multirow{2}{*}{$\begin{array}{c}\text { SE+CUP-GG+NR- } \\
\text { NMF+OU }\end{array}$} & \% STOI & 6.92 & 5.84 & 3.76 & 2.63 & 1.97 & 0.95 \\
\hline & STOI & 0.3338 & 0.5661 & 0.6528 & 0.7884 & 0.8383 & 0.9623 \\
\hline \multirow{2}{*}{$\begin{array}{c}\text { SE+CUP-GG+WR- } \\
\text { NMF+OU }\end{array}$} & \% STOI & 6.85 & 5.76 & 3.75 & 2.52 & 1.96 & 0.96 \\
\hline & STOI & 0.3336 & 0.5657 & 0.6528 & 0.7876 & 0.8382 & 0.9623 \\
\hline \multirow{2}{*}{$\mathrm{SE}+\mathrm{CUP}-\mathrm{GG}+\boldsymbol{\beta}-\mathrm{NMF}+\mathrm{OU}$} & \% STOI & 6.91 & 5.69 & 3.67 & 2.45 & 1.85 & 0.94 \\
\hline & STOI & 0.3338 & 0.5653 & 0.6523 & 0.7870 & 0.8373 & 0.9621 \\
\hline \multirow{2}{*}{ SE+CUP GG+NMF[9] } & \% STOI & 6.82 & 5.58 & 3.59 & 2.32 & 1.73 & 0.89 \\
\hline & STOI & 0.3335 & 0.5647 & 0.6518 & 0.7860 & 0.8363 & 0.9616 \\
\hline
\end{tabular}




\begin{tabular}{|c|c|c|c|c|c|c|c|}
\cline { 2 - 7 } NMF+OU[12] & \% STOI & 4.76 & 3.65 & 2.98 & 1.24 & 0.89 & 0.63 \\
\cline { 2 - 8 } & STOI & 0.3270 & 0.5544 & 0.6479 & 0.7777 & 0.8294 & 0.9592 \\
\hline \multirow{2}{*}{ CUP-GG[9] } & \% STOI & 4.97 & 3.78 & 3.21 & 1.78 & 0.49 & 0.43 \\
\cline { 2 - 8 } & STOI & 0.3277 & 0.5551 & 0.6494 & 0.7819 & 0.8261 & 0.9573 \\
\hline \multirow{2}{*}{ CUP-NG[9] } & \% STOI & 4.86 & 3.68 & 3.06 & 1.67 & 0.51 & 0.28 \\
& STOI & 0.3274 & 0.5546 & 0.6484 & 0.7810 & 0.8263 & 0.9559 \\
\hline \multirow{2}{*}{ CUP[8] } & \% STOI & 3.67 & 2.83 & 2.53 & 0.91 & 0.31 & 0.15 \\
\cline { 2 - 8 } & STOI & 0.3236 & 0.5500 & 0.6451 & 0.7752 & 0.8246 & 0.9546 \\
\hline \multirow{2}{*}{ WR NMF[14] } & \% STOI & 2.71 & 2.18 & 1.99 & 0.89 & 0.36 & 0.14 \\
\cline { 2 - 8 } & STOI & 0.3206 & 0.5465 & 0.6417 & 0.7750 & 0.8251 & 0.9545 \\
\hline \multirow{2}{*}{ NR NMF[14] } & \% STOI & 2.32 & 2.13 & 1.83 & 0.83 & 0.51 & 0.17 \\
\cline { 2 - 8 } & STOI & 0.3194 & 0.5463 & 0.6407 & 0.7746 & 0.8263 & 0.9548 \\
\hline \multirow{2}{*}{ PR NMF[11] } & \% STOI & 1.64 & 1.25 & 1.03 & 0.35 & 0.19 & 0.12 \\
\cline { 2 - 8 } & STOI & 0.3173 & 0.5416 & 0.6357 & 0.7708 & 0.8236 & 0.9543 \\
\hline \multirow{2}{*}{ HRNR-SPU[13] } & \% STOI & -2.34 & -2.24 & -1.34 & -1.19 & -1.18 & -1.11 \\
\cline { 2 - 8 } & STOI & 0.3048 & 0.5229 & 0.6207 & 0.7590 & 0.8124 & 0.9426 \\
\hline \multirow{2}{*}{ HRNR[5] } & \% STOI & -2.67 & -2.27 & -2.01 & -1.87 & -1.26 & -1.21 \\
\cline { 2 - 8 } & STOI & 0.3038 & 0.5227 & 0.6165 & 0.7538 & 0.8117 & 0.9416 \\
\hline
\end{tabular}

Table 20. Comparison of Average SNR, SDR, Seg Snr performance measures for the 30 speech signals corrupted by Car noise under Input SNRs of $10 \mathrm{~dB},-5 \mathrm{~dB}, 0 \mathrm{~dB}, 5 \mathrm{~dB}, 10 \mathrm{~dB}, 15 \mathrm{~dB}$.

\begin{tabular}{|c|c|c|c|c|c|c|c|}
\hline $\begin{array}{l}\text { Input SNR in dB } \\
\text { Method }\end{array}$ & $\begin{array}{l}\text { Performance } \\
\text { Measure }\end{array}$ & -10 & -5 & $\mathbf{0}$ & 5 & 10 & 15 \\
\hline \multirow{3}{*}{$\mathrm{SE}+\mathrm{CUP}-\mathrm{GG}+\mathrm{NR}-\mathrm{NMF}+\mathrm{OU}$} & SNR & 4.89 & 8.14 & 10.13 & 12.05 & 16.17 & 21.18 \\
\hline & SDR & 4.34 & 6.59 & 9.27 & 11.56 & 15.54 & 18.8 \\
\hline & Seg SNR & 3.78 & 6.46 & 7.69 & 11.12 & 14.98 & 17.35 \\
\hline \multirow{3}{*}{$\mathrm{SE}+\mathrm{CUP}-\mathrm{GG}+\mathrm{WR}-\mathrm{NMF}+\mathrm{OU}$} & SNR & 4.11 & 7.67 & 10.67 & 11.72 & 16.69 & 20.72 \\
\hline & SDR & 3.69 & 6.84 & 9.47 & 11.57 & 15.58 & 19.12 \\
\hline & Seg SNR & 3.58 & 6.02 & 7.84 & 10.31 & 15.02 & 16.87 \\
\hline \multirow{3}{*}{$\mathrm{SE}+\mathrm{CUP}-\mathrm{GG}+\boldsymbol{\beta}-\mathrm{NMF}+\mathrm{OU}$} & SNR & 4.58 & 7.12 & 10.32 & 11.47 & 15.68 & 20.14 \\
\hline & SDR & 4.08 & 6.57 & 9.05 & 10.89 & 14.87 & 18.45 \\
\hline & Seg SNR & 3.69 & 5.84 & 7.74 & 10.18 & 14.68 & 16.28 \\
\hline \multirow{3}{*}{ SE+CUP GG+NMF[9] } & SNR & 4.43 & 7.04 & 9.74 & 11.47 & 14.97 & 19.67 \\
\hline & SDR & 3.87 & 6.12 & 8.87 & 10.56 & 14.54 & 16.94 \\
\hline & Seg SNR & 3.45 & 5.68 & 7.45 & 9.75 & 13.42 & 15.67 \\
\hline \multirow{3}{*}{$\mathrm{NMF}+\mathrm{OU}[12]$} & SNR & 2.87 & 4.04 & 7.95 & 11.24 & 14.98 & 17.78 \\
\hline & SDR & 2.12 & 3.14 & 7.12 & 9.26 & 13.58 & 15.54 \\
\hline & Seg SNR & 1.48 & 1.96 & 6.84 & 8.64 & 12.46 & 14.67 \\
\hline \multirow{3}{*}{ CUP-GG[9] } & SNR & 4.25 & 6.84 & 8.94 & 10.94 & 15.68 & 19.42 \\
\hline & SDR & 3.88 & 6.82 & 8.78 & 10.72 & 13.95 & 16.32 \\
\hline & Seg SNR & 3.46 & 5.45 & 7.21 & 9.13 & 12.65 & 15.11 \\
\hline \multirow{3}{*}{ CUP-NG[9] } & SNR & 4.18 & 5.78 & 8.57 & 10.38 & 14.08 & 18.24 \\
\hline & SDR & 3.01 & 3.78 & 7.71 & 9.12 & 13.02 & 15.68 \\
\hline & Seg SNR & 2.13 & 4.48 & 6.68 & 8.58 & 12.08 & 14.13 \\
\hline \multirow{3}{*}{ CUP[8] } & SNR & 2.25 & 3.68 & 7.74 & 9.31 & 13.34 & 17.11 \\
\hline & SDR & 2.65 & 3.05 & 6.69 & 8.65 & 12.74 & 14.87 \\
\hline & Seg SNR & 1.68 & 2.12 & 5.98 & 7.85 & 10.89 & 13.87 \\
\hline \multirow{3}{*}{ NR-NMF[14] } & SNR & 1.17 & 4.85 & 5.12 & 9.47 & 15.76 & 18.85 \\
\hline & SDR & 0.89 & 2.84 & 4.78 & 10.57 & 14.54 & 18.12 \\
\hline & Seg SNR & -3.24 & -0.91 & 3.25 & 6.77 & 10.84 & 13.68 \\
\hline \multirow{3}{*}{ WR-NMF[14] } & SNR & 0.64 & 3.12 & 4.57 & 9.45 & 15.02 & 18.42 \\
\hline & SDR & 0.18 & 2.08 & 4.14 & 9.21 & 13.84 & 17.75 \\
\hline & Seg SNR & -3.78 & -1.54 & 2.52 & 4.98 & 10.67 & 13.14 \\
\hline \multirow{3}{*}{ PR NMF [11] } & SNR & 0.11 & 1.92 & 4.21 & 8.54 & 12.85 & 16.96 \\
\hline & SDR & -2.17 & 1.28 & 3.12 & 7.02 & 12.21 & 15.34 \\
\hline & Seg SNR & -4.62 & -2.51 & 1.31 & 3.85 & 9.24 & 11.98 \\
\hline \multirow{3}{*}{ HRNR-SPU [13] } & SNR & 1.94 & 1.81 & 4.32 & 6.97 & 12.42 & 17.43 \\
\hline & SDR & 1.21 & 1.62 & 2.61 & 5.45 & 11.26 & 15.68 \\
\hline & Seg SNR & 0.31 & 1.25 & 2.11 & 2.68 & 6.54 & 8.96 \\
\hline \multirow{3}{*}{ HRNR[5] } & SNR & 1.17 & 1.62 & 2.24 & 6.45 & 10.85 & 15.41 \\
\hline & SDR & 0.84 & 1.34 & 2.28 & 5.42 & 10.53 & 15.14 \\
\hline & Seg SNR & 0.1 & 0.87 & 1.66 & 2.28 & 6.14 & 9.24 \\
\hline
\end{tabular}


Table 21. Comparison of Average PESQ, $\triangle \mathrm{PESQ}$ performance measures for the 30 speech signals corrupted by F16 noise under Input SNRs of -10 $\mathrm{dB},-5 \mathrm{~dB}, 0 \mathrm{~dB}, 5 \mathrm{~dB}, 10 \mathrm{~dB}, 15 \mathrm{~dB}$.

\begin{tabular}{|c|c|c|c|c|c|c|c|}
\hline $\begin{array}{l}\text { Input SNR in dB } \\
\text { Method }\end{array}$ & $\begin{array}{l}\text { Performance } \\
\text { Measure }\end{array}$ & -10 & -5 & $\mathbf{0}$ & 5 & 10 & 15 \\
\hline Without Processing & PESQ & 1.17 & 1.61 & 1.87 & 2.05 & 2.38 & 2.62 \\
\hline \multirow{2}{*}{$\begin{array}{c}\text { SE+CUP-GG+NR- } \\
\text { NMF+OU }\end{array}$} & PESQ & 1.38 & 2.14 & 2.69 & 2.9 & 3.2 & 3.37 \\
\hline & $\triangle$ PESQ & 0.21 & 0.53 & 0.82 & 0.85 & 0.82 & 0.75 \\
\hline \multirow{2}{*}{$\begin{array}{c}\text { SE+CUP-GG+WR- } \\
\text { NMF+OU }\end{array}$} & PESQ & 1.37 & 2.09 & 2.63 & 2.87 & 3.17 & 3.33 \\
\hline & $\triangle$ PESQ & 0.2 & 0.48 & 0.76 & 0.82 & 0.79 & 0.71 \\
\hline \multirow{2}{*}{$\mathrm{SE}+\mathrm{CUP}-\mathrm{GG}+\boldsymbol{\beta}-\mathrm{NMF}+\mathrm{OU}$} & PESQ & 1.35 & 2.07 & 2.56 & 2.81 & 3.13 & 3.31 \\
\hline & $\triangle$ PESQ & 0.18 & 0.46 & 0.69 & 0.76 & 0.75 & 0.69 \\
\hline \multirow{2}{*}{ SE+CUP GG+NMF[9] } & PESQ & 1.33 & 2 & 2.51 & 2.76 & 3.01 & 3.18 \\
\hline & $\triangle$ PESQ & 0.16 & 0.39 & 0.64 & 0.71 & 0.63 & 0.56 \\
\hline \multirow{2}{*}{$\mathrm{NMF}+\mathrm{OU}[12]$} & PESQ & 1.29 & 1.93 & 2.46 & 2.71 & 2.95 & 3.11 \\
\hline & $\triangle$ PESQ & 0.12 & 0.32 & 0.59 & 0.66 & 0.57 & 0.49 \\
\hline \multirow{2}{*}{ CUP-GG[9] } & PESQ & 1.3 & 1.95 & 2.49 & 2.73 & 2.97 & 3.13 \\
\hline & $\triangle$ PESQ & 0.13 & 0.34 & 0.62 & 0.68 & 0.59 & 0.51 \\
\hline \multirow{2}{*}{ CUP-NG[9] } & PESQ & 1.28 & 1.9 & 2.36 & 2.61 & 2.92 & 3.09 \\
\hline & $\triangle$ PESQ & 0.11 & 0.29 & 0.49 & 0.56 & 0.54 & 0.47 \\
\hline \multirow{2}{*}{ CUP[8] } & PESQ & 1.22 & 1.87 & 2.38 & 2.58 & 2.84 & 3.05 \\
\hline & $\triangle$ PESQ & 0.05 & 0.26 & 0.51 & 0.53 & 0.46 & 0.43 \\
\hline \multirow{2}{*}{ WR NMF[14] } & PESQ & 1.33 & 1.96 & 2.44 & 2.68 & 2.96 & 3.16 \\
\hline & $\triangle$ PESQ & 0.16 & 0.35 & 0.57 & 0.63 & 0.58 & 0.54 \\
\hline \multirow{2}{*}{ NR NMF[14] } & PESQ & 1.31 & 1.98 & 2.38 & 2.62 & 2.97 & 3.13 \\
\hline & $\triangle$ PESQ & 0.14 & 0.37 & 0.51 & 0.57 & 0.59 & 0.51 \\
\hline \multirow{2}{*}{ PR NMF[11] } & PESQ & 1.28 & 1.83 & 2.16 & 2.43 & 2.79 & 2.98 \\
\hline & $\triangle$ PESQ & 0.11 & 0.22 & 0.29 & 0.38 & 0.41 & 0.36 \\
\hline \multirow{2}{*}{ HRNR-SPU[13] } & PESQ & 1.24 & 1.69 & 2.12 & 2.34 & 2.7 & 2.9 \\
\hline & $\triangle$ PESQ & 0.07 & 0.08 & 0.25 & 0.29 & 0.32 & 0.28 \\
\hline \multirow{2}{*}{ HRNR[5] } & PESQ & 1.04 & 1.71 & 2.05 & 2.24 & 2.55 & 2.77 \\
\hline & $\triangle$ PESQ & -0.13 & 0.1 & 0.18 & 0.19 & 0.17 & 0.15 \\
\hline
\end{tabular}

In table 21, the Comparison of Average PESQ, $\triangle \mathrm{PESQ}$ performance measures for the 30 speech signals corrupted by F16 noise under input SNRs of $-10 \mathrm{~dB},-5 \mathrm{~dB}, 0 \mathrm{~dB}, 5$ $\mathrm{dB}, 10 \mathrm{~dB}, 15 \mathrm{~dB}$ is listed. It is observed that the, $\mathrm{SE}+$ CUP-GG + NR-NMF + OU provides improved performance than to all compared methods and it provides a $\triangle$ PESQ value of 0.82 for the input signal corrupted under F16 noise at OdB SNR. It is observed that $\mathrm{SE}+\mathrm{CUP}-\mathrm{GG}+\mathrm{NMF}$ approach provides an PESQ improvement of 0.64. Also it is observed that CUP-GG estimators provides an improved $\triangle \mathrm{PESQ}$ value of 0.62 compared to posterior WR-NMF of 0.57 at $0 \mathrm{~dB}$ input SNR. It is noted that the Bayesian estimators shown comparable improvement under low SNRs than to traditional NMF approach. Also noted that combination of Bayesian estimator with NMF approach provides improved results in case of F16 noise.

In table 22, the Comparison of Average STOI, \%STOI performance measures for the 30 speech signals corrupted by F16 noise under Input SNRs of $-10 \mathrm{~dB},-5 \mathrm{~dB}, 0 \mathrm{~dB}, 5$ $\mathrm{dB}, 10 \mathrm{~dB}, 15 \mathrm{~dB}$ is listed. It is observed that \% STOI improvement of STOI values for SE + CUP - GG + NR $\mathrm{NMF}+\mathrm{OU}$ approach is $6.45,5.51,3.41,2.23,1.59,0.69$ and for CUP-GG estimator is 3.95, 3.15, 2.45, 1.75, 0.78, 0.28 and for WR-NMF approach is $2.54,2.11,1.82,0.75$, 0.34, 0.09 at input SNRs of $-10 \mathrm{~dB},-5 \mathrm{~dB}, 0 \mathrm{~dB}, 5 \mathrm{~dB}, 10$ $\mathrm{dB}, 15 \mathrm{~dB}$ respectively. It is noted that at $-10 \mathrm{~dB},-5 \mathrm{~dB}$ SNRs there is significant improvement in SE+CUPGG+NMF based approaches than to all other methods.

In Table 23, the Comparison of Average SNR, SDR, Seg SNR performance measures for the 30 speech signals corrupted by F16 noise under Input SNRs of $-10 \mathrm{~dB}$, $5 \mathrm{~dB}, 0 \mathrm{~dB}, 5 \mathrm{~dB}, 10 \mathrm{~dB}, 15 \mathrm{~dB}$ is listed. The proposed method SE+CUP-GG+NR-NMF+OU provides an SNR of $4.21,7.57,9.89,11.78,15.68,19.85$, and for SE+CUPGG+NMF approach is 3.41, 6.12, 8.11, 9.48, 13.4, 18.52 . The NR-NMF approach provides an SNR of 1.69, 3.84, $6.36,8.75,13.45,15.97$.

Table 22. Comparison of Average STOI, \%STOI performance measures for the 30 speech signals corrupted by F16 noise under Input SNRs of -10 dB, $-5 \mathrm{~dB}, 0 \mathrm{~dB}, 5 \mathrm{~dB}, 10 \mathrm{~dB}, 15 \mathrm{~dB}$.

\begin{tabular}{|c|c|c|c|c|c|c|c|}
\hline $\begin{array}{l}\text { Input SNR in dB } \\
\text { Method }\end{array}$ & $\begin{array}{l}\text { Performance } \\
\text { Measure }\end{array}$ & -10 & -5 & $\mathbf{0}$ & 5 & 10 & 15 \\
\hline Without Processing & STOI & 0.2912 & 0.5012 & 0.5963 & 0.7256 & 0.8124 & 0.8874 \\
\hline \multirow{2}{*}{$\mathrm{SE}+\mathrm{CUP}-\mathrm{GG}+\mathrm{NR}-\mathrm{NMF}+\mathrm{OU}$} & \% STOI & 6.45 & 5.51 & 3.41 & 2.23 & 1.59 & 0.69 \\
\hline & STOI & 0.3099 & 0.5288 & 0.6166 & 0.7417 & 0.8253 & 0.8935 \\
\hline \multirow{2}{*}{ SE+CUP-GG+WR-NMF+OU } & \% STOI & 6.37 & 5.36 & 3.31 & 2.11 & 1.48 & 0.61 \\
\hline & STOI & 0.3097 & 0.5281 & 0.6160 & 0.7409 & 0.8244 & 0.8928 \\
\hline \multirow{2}{*}{$\mathrm{SE}+\mathrm{CUP}-\mathrm{GG}+\boldsymbol{\beta}-\mathrm{NMF}+\mathrm{OU}$} & \% STOI & 6.27 & 5.11 & 3.14 & 2.06 & 1.45 & 0.58 \\
\hline & STOI & 0.3094 & 0.5268 & 0.6150 & 0.7405 & 0.8242 & 0.8925 \\
\hline \multirow{2}{*}{ SE+CUP GG+NMF[9] } & \% STOI & 6.04 & 5.14 & 2.98 & 1.89 & 1.42 & 0.54 \\
\hline & STOI & 0.3087 & 0.5269 & 0.6140 & 0.7393 & 0.8239 & 0.8922 \\
\hline
\end{tabular}




\begin{tabular}{|c|c|c|c|c|c|c|c|}
\cline { 2 - 7 } \multirow{4}{*}{ NMF+OU[12] } & \% STOI & 3.95 & 3.11 & 2.49 & 0.88 & 0.59 & 0.41 \\
\cline { 2 - 8 } & STOI & 0.3027 & 0.5167 & 0.6111 & 0.7319 & 0.8171 & 0.8910 \\
\hline \multirow{2}{*}{ CUP-GG[9] } & \% STOI & 3.95 & 3.15 & 2.45 & 1.75 & 0.78 & 0.28 \\
\cline { 2 - 8 } & STOI & 0.3027 & 0.5169 & 0.6109 & 0.7383 & 0.8187 & 0.8898 \\
\hline \multirow{2}{*}{ CUP-NG[9] } & \% STOI & 3.54 & 2.84 & 2.93 & 1.06 & 0.58 & 0.15 \\
\cline { 2 - 8 } & STOI & 0.3015 & 0.5154 & 0.6137 & 0.7332 & 0.8171 & 0.8887 \\
\hline \multirow{2}{*}{ CUP[8] } & \% STOI & 2.91 & 2.47 & 1.79 & 0.58 & 0.18 & 0.06 \\
\cline { 2 - 8 } & STOI & 0.2996 & 0.5135 & 0.6069 & 0.7298 & 0.8138 & 0.8879 \\
\hline \multirow{2}{*}{ WR NMF[14] } & \% STOI & 2.54 & 2.11 & 1.82 & 0.75 & 0.34 & 0.09 \\
\cline { 2 - 8 } & STOI & 0.2985 & 0.5117 & 0.6071 & 0.7310 & 0.8151 & 0.8882 \\
\hline \multirow{2}{*}{ NR NMF[14] } & \% STOI & 2.12 & 1.85 & 1.56 & 0.59 & 0.19 & 0.11 \\
\cline { 2 - 8 } & STOI & 0.2973 & 0.5104 & 0.6056 & 0.7298 & 0.8139 & 0.8883 \\
\hline \multirow{2}{*}{ PR NMF[11] } & \% STOI & 1.54 & 1.26 & 0.85 & 0.18 & 0.08 & 0.07 \\
\cline { 2 - 8 } & STOI & 0.2956 & 0.5075 & 0.6013 & 0.7269 & 0.8130 & 0.8880 \\
\hline \multirow{2}{*}{ HRNR-SPU[13] } & \% STOI & -2.26 & -2.11 & -1.35 & -1.65 & -1.21 & -1.23 \\
\cline { 2 - 8 } & STOI & 0.2846 & 0.4906 & 0.5882 & 0.7136 & 0.8025 & 0.8764 \\
\hline \multirow{2}{*}{ HRNR[5] } & \% STOI & -2.78 & -2.17 & -2.09 & -1.31 & -1.43 & -1.5 \\
\cline { 2 - 8 } & STOI & 0.2831 & 0.4903 & 0.5838 & 0.7161 & 0.8007 & 0.8741 \\
\hline
\end{tabular}

Table 23. Comparison of Average SNR, SDR, Seg SNR performance measures for the 30 speech signals corrupted by F16 noise under Input SNRs of $-10 \mathrm{~dB},-5 \mathrm{~dB}, 0 \mathrm{~dB}, 5 \mathrm{~dB}, 10 \mathrm{~dB}, 15 \mathrm{~dB}$

\begin{tabular}{|c|c|c|c|c|c|c|c|}
\hline $\begin{array}{l}\text { Input SNR in dB } \\
\text { Method }\end{array}$ & $\begin{array}{l}\text { Performance } \\
\text { Measure }\end{array}$ & -10 & -5 & $\mathbf{0}$ & 5 & 10 & 15 \\
\hline \multirow{3}{*}{$\mathrm{SE}+\mathrm{CUP}-\mathrm{GG}+\mathrm{NR}-\mathrm{NMF}+\mathrm{OU}$} & SNR & 4.21 & 7.57 & 9.89 & 11.78 & 15.68 & 19.85 \\
\hline & SDR & 3.91 & 6.34 & 8.85 & 10.12 & 13.54 & 18.02 \\
\hline & Seg SNR & 3.84 & 5.92 & 7.54 & 9.85 & 12.86 & 16.84 \\
\hline \multirow{3}{*}{$\mathrm{SE}+\mathrm{CUP}-\mathrm{GG}+\mathrm{WR}-\mathrm{NMF}+\mathrm{OU}$} & SNR & 4.18 & 6.48 & 8.92 & 10.29 & 13.75 & 19.89 \\
\hline & SDR & 3.48 & 5.69 & 8.67 & 10.08 & 13.21 & 17.98 \\
\hline & Seg SNR & 3.37 & 5.52 & 7.46 & 9.85 & 12.95 & 16.39 \\
\hline \multirow{3}{*}{$\mathrm{SE}+\mathrm{CUP}-\mathrm{GG}+\boldsymbol{\beta}-\mathrm{NMF}+\mathrm{OU}$} & SNR & 3.84 & 6.74 & 8.75 & 10.12 & 13.54 & 18.54 \\
\hline & SDR & 3.49 & 5.68 & 8.42 & 9.58 & 13.24 & 17.62 \\
\hline & Seg SNR & 3.32 & 5.73 & 7.42 & 9.92 & 12.97 & 15.84 \\
\hline \multirow{3}{*}{ SE+CUP GG+NMF[9] } & SNR & 3.41 & 6.12 & 8.11 & 9.48 & 13.44 & 18.52 \\
\hline & SDR & 3.18 & 6.02 & 8.13 & 9.37 & 13.27 & 17.23 \\
\hline & Seg SNR & 2.97 & 5.12 & 6.78 & 9.02 & 12.97 & 15.47 \\
\hline \multirow{3}{*}{$\mathrm{NMF}+\mathrm{OU}[12]$} & SNR & 3.12 & 3.76 & 7.32 & 8.75 & 12.82 & $17 . .31$ \\
\hline & SDR & 2.81 & 3.36 & 7.37 & 8.45 & 11.97 & 14.21 \\
\hline & Seg SNR & 2.35 & 4.08 & 6.04 & 8.24 & 11.48 & 13.74 \\
\hline \multirow{3}{*}{ CUP-GG[9] } & SNR & 3.14 & 5.85 & 7.74 & 8.95 & 12.04 & 17.84 \\
\hline & SDR & 2.84 & 3.42 & 7.68 & 8.82 & 12.75 & 15.98 \\
\hline & Seg SNR & 2.86 & 5.24 & 6.89 & 8.56 & 12.35 & 14.82 \\
\hline \multirow{3}{*}{ CUP-NG[9] } & SNR & 3.05 & 4.48 & 7.75 & 9.48 & 13.02 & 17.69 \\
\hline & SDR & 2.86 & 3.45 & 7.38 & 8.75 & 12.62 & 15.34 \\
\hline & Seg SNR & 2.16 & 4.01 & 6.34 & 8.37 & 11.25 & 13.59 \\
\hline \multirow{3}{*}{ CUP[8] } & SNR & 3.21 & 3.56 & 7.57 & 9.02 & 12.75 & 17.04 \\
\hline & SDR & 2.62 & 3.25 & 7.16 & 8.68 & 12.54 & 15.32 \\
\hline & Seg SNR & 1.59 & 2.27 & 5.82 & 8.04 & 11.14 & 13.26 \\
\hline \multirow{3}{*}{ NR-NMF[14] } & SNR & 1.69 & 3.84 & 6.36 & 8.75 & 13.45 & 15.97 \\
\hline & SDR & 0.84 & 2.61 & 5.83 & 7.95 & 12.34 & 14.95 \\
\hline & Seg SNR & -2.43 & -0.74 & 2.98 & 6.34 & 10.79 & 13.12 \\
\hline \multirow{3}{*}{ WR-NMF[14] } & SNR & 1.28 & 3.72 & 5.92 & 8.67 & 12.88 & 15.67 \\
\hline & SDR & 0.25 & 2.36 & 5.84 & 7.86 & 11.69 & 15.12 \\
\hline & Seg SNR & -3.02 & -0.98 & 2.57 & 5.32 & 10.85 & 13.64 \\
\hline \multirow{3}{*}{ PR NMF [11] } & SNR & 0.12 & 2.21 & 4.34 & 7.68 & 11.46 & 15.54 \\
\hline & SDR & -2.11 & 1.32 & 3.12 & 6.98 & 10.21 & 13.02 \\
\hline & Seg SNR & -3.42 & -1.23 & 2.31 & 4.92 & 9.62 & 12.58 \\
\hline \multirow{3}{*}{ HRNR-SPU [13] } & SNR & -3.95 & -1.26 & 0.12 & 5.36 & 11.67 & 15.23 \\
\hline & SDR & -5.87 & -3.59 & -0.12 & 3.14 & 6.45 & 12.05 \\
\hline & Seg SNR & -8.86 & -3.54 & -1.24 & 2.86 & 6.95 & 9.87 \\
\hline \multirow{3}{*}{ HRNR[5] } & SNR & -5.01 & -1.76 & -0.02 & 5.52 & 10.72 & 12.41 \\
\hline & SDR & -7.24 & -2.66 & -0.23 & 2.81 & 9.68 & 11.54 \\
\hline & Seg SNR & -9.11 & -4.23 & -1.12 & 2.37 & 5.93 & 9.45 \\
\hline
\end{tabular}


Table 24. Comparison of Average PESQ, $\triangle$ PESQ performance measures for the 30 speech signals corrupted by M109 noise under Input SNRs of -10 $\mathrm{dB},-5 \mathrm{~dB}, 0 \mathrm{~dB}, 5 \mathrm{~dB}, 10 \mathrm{~dB}, 15 \mathrm{~dB}$.

\begin{tabular}{|c|c|c|c|c|c|c|c|}
\hline $\begin{array}{l}\text { Input SNR in dB } \\
\text { Method }\end{array}$ & $\begin{array}{l}\text { Performance } \\
\text { Measure }\end{array}$ & -10 & -5 & $\mathbf{0}$ & 5 & 10 & 15 \\
\hline Without Processing & PESQ & 1.39 & 1.72 & 1.9 & 2.28 & 2.52 & 2.91 \\
\hline \multirow{2}{*}{$\mathrm{SE}+\mathrm{CUP}-\mathrm{GG}+\mathrm{NR}-\mathrm{NMF}+\mathrm{OU}$} & PESQ & 1.61 & 2.26 & 2.78 & 3.19 & 3.38 & 3.73 \\
\hline & $\triangle$ PESQ & 0.22 & 0.54 & 0.88 & 0.91 & 0.86 & 0.82 \\
\hline \multirow{2}{*}{ SE+CUP-GG+WR-NMF+OU } & PESQ & 1.58 & 2.21 & 2.76 & 3.17 & 3.34 & 3.72 \\
\hline & $\triangle$ PESQ & 0.19 & 0.49 & 0.86 & 0.89 & 0.82 & 0.81 \\
\hline \multirow{2}{*}{$\mathrm{SE}+\mathrm{CUP}-\mathrm{GG}+\boldsymbol{\beta}-\mathrm{NMF}+\mathrm{OU}$} & PESQ & 1.56 & 2.15 & 2.71 & 3.13 & 3.35 & 3.69 \\
\hline & $\triangle$ PESQ & 0.17 & 0.43 & 0.81 & 0.85 & 0.83 & 0.78 \\
\hline \multirow{2}{*}{ SE+CUP GG+NMF[9] } & PESQ & 1.54 & 2.1 & 2.67 & 3.07 & 3.3 & 3.62 \\
\hline & $\triangle$ PESQ & 0.15 & 0.38 & 0.77 & 0.79 & 0.78 & 0.71 \\
\hline \multirow{2}{*}{$\mathrm{NMF}+\mathrm{OU}[12]$} & PESQ & 1.51 & 2.01 & 2.52 & 2.88 & 3.1 & 3.45 \\
\hline & $\triangle$ PESQ & 0.12 & 0.29 & 0.62 & 0.6 & 0.58 & 0.54 \\
\hline \multirow{2}{*}{ CUP-GG[9] } & PESQ & 1.51 & 2.08 & 2.55 & 2.95 & 3.13 & 3.51 \\
\hline & $\triangle$ PESQ & 0.12 & 0.36 & 0.65 & 0.67 & 0.61 & 0.6 \\
\hline \multirow{2}{*}{ CUP-NG[9] } & PESQ & 1.48 & 2 & 2.43 & 2.8 & 2.99 & 3.37 \\
\hline & $\triangle$ PESQ & 0.09 & 0.28 & 0.53 & 0.52 & 0.47 & 0.46 \\
\hline \multirow{2}{*}{ CUP[8] } & PESQ & 1.4 & 1.95 & 2.44 & 2.74 & 3 & 3.33 \\
\hline & $\triangle$ PESQ & 0.01 & 0.23 & 0.54 & 0.46 & 0.48 & 0.42 \\
\hline \multirow{2}{*}{ WR NMF[14] } & PESQ & 1.5 & 2.06 & 2.48 & 2.89 & 3.15 & 3.47 \\
\hline & $\triangle$ PESQ & 0.11 & 0.34 & 0.58 & 0.61 & 0.63 & 0.56 \\
\hline \multirow{2}{*}{ NR NMF[14] } & PESQ & 1.51 & 2.08 & 2.46 & 2.87 & 3.13 & 3.47 \\
\hline & $\triangle$ PESQ & 0.12 & 0.36 & 0.56 & 0.59 & 0.61 & 0.56 \\
\hline \multirow{2}{*}{ PR NMF[11] } & PESQ & 1.48 & 1.94 & 2.22 & 2.69 & 2.94 & 3.28 \\
\hline & $\triangle$ PESQ & 0.09 & 0.22 & 0.32 & 0.41 & 0.42 & 0.37 \\
\hline \multirow{2}{*}{ HRNR-SPU[13] } & PESQ & 1.45 & 1.81 & 2.18 & 2.57 & 2.82 & 3.16 \\
\hline & $\triangle$ PESQ & 0.06 & 0.09 & 0.28 & 0.29 & 0.3 & 0.25 \\
\hline \multirow{2}{*}{ HRNR[5] } & PESQ & 1.28 & 1.79 & 2.06 & 2.49 & 2.72 & 3.08 \\
\hline & $\triangle$ PESQ & -0.11 & 0.07 & 0.16 & 0.21 & 0.2 & 0.17 \\
\hline
\end{tabular}

In table 25, the Comparison of Average PESQ, $\triangle \mathrm{PESQ}$ performance measures for the 30 speech signals corrupted by M109 noise under input SNRs of $-10 \mathrm{~dB},-5 \mathrm{~dB}, 0 \mathrm{~dB}, 5$ $\mathrm{dB}, 10 \mathrm{~dB}, 15 \mathrm{~dB}$ is listed. It is observed that the, $\mathrm{SE}+$ CUP-GG + NR-NMF + OU provides improved performance than to all compared methods and it provides a $\triangle \mathrm{PESQ}$ value of 0.88 for the input signal corrupted under M109 noise at 0dB SNR. It is observed that SE+CUP-GG + NMF approach provides an PESQ improvement of 0.77. Also it is observed that CUP-GG estimators provides an improved $\triangle \mathrm{PESQ}$ value of 0.65 compared to posterior WR-NMF of 0.58 at $0 \mathrm{~dB}$ input SNR. It is noted that the Bayesian estimators shown comparable improvement under low SNRs than to traditional NMF approach. Also it is noted that combination of Bayesian estimator with NMF approach provides improved results in case of M109 noise.

In table 26, the Comparison of Average STOI, \%STOI performance measures for the 30 speech signals corrupted by M109 noise under Input SNRs of $-10 \mathrm{~dB},-5 \mathrm{~dB}, 0 \mathrm{~dB}, 5$ $\mathrm{dB}, 10 \mathrm{~dB}, 15 \mathrm{~dB}$ is listed. It is observed that \% STOI improvement of STOI values for SE + CUP - GG + NR $\mathrm{NMF}+\mathrm{OU}$ approach is $6.23,5.46,3.44,2.26,1.61,0.65$ and for CUP-GG estimator is 3.91, 3.12, 2.36, 1.85, 0.84, 0.31 and for WR-NMF approach is $2.49,1.98,1.75,0.71$, $0.28,0.12$ at input SNRs of $-10 \mathrm{~dB},-5 \mathrm{~dB}, 0 \mathrm{~dB}, 5 \mathrm{~dB}, 10$ $\mathrm{dB}, 15 \mathrm{~dB}$ respectively. It is noted that at $-10 \mathrm{~dB},-5 \mathrm{~dB}$ SNRs there is significant improvement in SE+CUPGG+NMF based approaches than to all other methods.

In table 27, the Comparison of Average SNR, SDR, Seg SNR performance measures for the 30 speech signals corrupted by M109 noise under Input SNRs of -10 dB, $5 \mathrm{~dB}, 0 \mathrm{~dB}, 5 \mathrm{~dB}, 10 \mathrm{~dB}, 15 \mathrm{~dB}$ is listed. The proposed method SE+CUP-GG+NR-NMF+OU provides an SNR of 3.98, 7.42, 9.75, 11.69, 15.72, 20.04, and for SE+CUPGG+NMF approach is 3.51, 6.25, 8.13, 9.61, 13.52, 18.78. The NR-NMF approach provides an SNR of 3.51, $6.25,8.13,9.61,13.52,18.78$

Table 25. Comparison of Average STOI, \% STOI performance measures for the 30 speech signals corrupted by M109 noise under Input SNRs of -10 $\mathrm{dB},-5 \mathrm{~dB}, 0 \mathrm{~dB}, 5 \mathrm{~dB}, 10 \mathrm{~dB}, 15 \mathrm{~dB}$

\begin{tabular}{|c|c|c|c|c|c|c|c|}
\hline $\begin{array}{l}\text { Input SNR in dB } \\
\text { Method }\end{array}$ & $\begin{array}{l}\text { Performance } \\
\text { Measure }\end{array}$ & -10 & -5 & $\mathbf{0}$ & 5 & 10 & 15 \\
\hline Without Processing & STOI & 0.2826 & 0.4965 & 0.5982 & 0.7145 & 0.8147 & 0.8712 \\
\hline \multirow{2}{*}{$\begin{array}{c}\mathrm{SE}+\mathrm{CUP}-\mathrm{GG}+\mathrm{NR}- \\
\mathrm{NMF}+\mathrm{OU}\end{array}$} & $\%$ STOI & 6.23 & 5.46 & 3.44 & 2.26 & 1.61 & 0.65 \\
\hline & STOI & 0.3002 & 0.5236 & 0.6187 & 0.7306 & 0.8278 & 0.8768 \\
\hline \multirow{2}{*}{$\begin{array}{c}\text { SE+CUP-GG+WR- } \\
\text { NMF+OU }\end{array}$} & $\%$ STOI & 6.21 & 5.14 & 3.12 & 2.08 & 1.42 & 0.56 \\
\hline & STOI & 0.3001 & 0.5220 & 0.6168 & 0.7293 & 0.8262 & 0.8761 \\
\hline \multirow{2}{*}{$\mathrm{SE}+\mathrm{CUP}-\mathrm{GG}+\boldsymbol{\beta}-\mathrm{NMF}+\mathrm{OU}$} & \% STOI & 6.14 & 5.04 & 3.09 & 1.96 & 1.41 & 0.57 \\
\hline & STOI & 0.2999 & 0.5215 & 0.6166 & 0.7285 & 0.8261 & 0.8761 \\
\hline \multirow{2}{*}{ SE+CUP GG+NMF[9] } & \% STOI & 5.96 & 5.12 & 2.78 & 1.82 & 1.39 & 0.51 \\
\hline & STOI & 0.2994 & 0.5219 & 0.6148 & 0.7275 & 0.8260 & 0.8756 \\
\hline
\end{tabular}




\begin{tabular}{|c|c|c|c|c|c|c|c|}
\cline { 2 - 7 } NMF+OU[12] & \% STOI & 3.89 & 3.01 & 2.51 & 0.92 & 0.58 & 0.43 \\
\cline { 2 - 8 } & STOI & 0.2933 & 0.5114 & 0.6132 & 0.7211 & 0.8194 & 0.8749 \\
\hline \multirow{2}{*}{ CUP-GG[9] } & \% STOI & 3.91 & 3.12 & 2.36 & 1.85 & 0.84 & 0.31 \\
\cline { 2 - 8 } & STOI & 0.2936 & 0.5119 & 0.6123 & 0.7277 & 0.8215 & 0.8739 \\
\hline \multirow{2}{*}{ CUP-NG[9] } & \% STOI & 3.51 & 2.89 & 2.91 & 1.12 & 0.61 & 0.18 \\
\cline { 2 - 8 } & STOI & 0.2925 & 0.51084 & 0.6156 & 0.7225 & 0.8196 & 0.8727 \\
\hline \multirow{2}{*}{ CUP[8] } & \% STOI & 2.81 & 2.42 & 1.74 & 0.56 & 0.14 & 0.03 \\
\cline { 2 - 8 } & STOI & 0.2905 & 0.5085 & 0.6086 & 0.7185 & 0.8158 & 0.8714 \\
\hline \multirow{2}{*}{ WR NMF[14] } & \% STOI & 2.49 & 1.98 & 1.75 & 0.71 & 0.28 & 0.12 \\
\cline { 2 - 8 } & STOI & 0.2896 & 0.5063 & 0.6086 & 0.7195 & 0.8169 & 0.8722 \\
\hline \multirow{2}{*}{ NR NMF[14] } & \% STOI & 2.15 & 1.79 & 1.52 & 0.56 & 0.21 & 0.13 \\
\cline { 2 - 8 } & STOI & 0.2886 & 0.5054 & 0.6073 & 0.7185 & 0.8164 & 0.8723 \\
\hline \multirow{2}{*}{ PR NMF[11] } & \% STOI & 1.53 & 1.22 & 0.89 & 0.21 & 0.11 & 0.06 \\
\cline { 2 - 8 } & STOI & 0.2869 & 0.5025 & 0.6035 & 0.716 & 0.8155 & 0.8717 \\
\hline \multirow{2}{*}{ HRNR-SPU[13] } & \% STOI & -2.18 & -2.11 & -1.43 & -1.57 & -1.24 & -1.19 \\
\cline { 2 - 8 } & STOI & 0.2764 & 0.4860 & 0.5896 & 0.7033 & 0.8045 & 0.8608 \\
\hline \multirow{2}{*}{ HRNR[5] } & \% STOI & -2.65 & -2.14 & -2.01 & -1.21 & -1.36 & -1.57 \\
\cline { 2 - 8 } & STOI & 0.2751 & 0.4858 & 0.5861 & 0.7058 & 0.8036 & 0.8575 \\
\hline
\end{tabular}

Table 26. Comparison of Average SNR, SDR, Seg SNR performance measures for the 30 speech signals corrupted by M109 noise under Input SNRs of $-10 \mathrm{~dB},-5 \mathrm{~dB}, 0 \mathrm{~dB}, 5 \mathrm{~dB}, 10 \mathrm{~dB}, 15 \mathrm{~dB}$

\begin{tabular}{|c|c|c|c|c|c|c|c|}
\hline Method Input SNR in dB & $\begin{array}{l}\text { Performance } \\
\text { Measure }\end{array}$ & -10 & -5 & $\mathbf{0}$ & 5 & 10 & 15 \\
\hline \multirow{3}{*}{$\mathrm{SE}+\mathrm{CUP}-\mathrm{GG}+\mathrm{NR}-\mathrm{NMF}+\mathrm{OU}$} & SNR & 3.98 & 7.42 & 9.75 & 11.69 & 15.72 & 20.04 \\
\hline & SDR & 3.87 & 6.27 & 8.73 & 10.25 & 13.68 & 18.24 \\
\hline & Seg SNR & 3.67 & 5.84 & 7.43 & 9.74 & 12.75 & 16.75 \\
\hline \multirow{3}{*}{$\mathrm{SE}+\mathrm{CUP}-\mathrm{GG}+\mathrm{WR}-\mathrm{NMF}+\mathrm{OU}$} & SNR & 4.09 & 6.37 & 8.86 & 10.06 & 13.64 & 19.75 \\
\hline & SDR & 3.34 & 5.57 & 8.53 & 9.98 & 13.14 & 17.85 \\
\hline & Seg SNR & 3.32 & 5.41 & 7.38 & 9.76 & 12.87 & 16.27 \\
\hline \multirow{3}{*}{$\mathrm{SE}+\mathrm{CUP}-\mathrm{GG}+\boldsymbol{\beta}-\mathrm{NMF}+\mathrm{OU}$} & SNR & 3.89 & 6.84 & 8.79 & 10.25 & 13.67 & 18.72 \\
\hline & SDR & 3.36 & 6.45 & 8.34 & 9.42 & 13.11 & 17.39 \\
\hline & Seg SNR & 3.24 & 5.62 & 7.28 & 9.85 & 12.82 & 15.71 \\
\hline \multirow{3}{*}{ SE+CUP GG+NMF[9] } & SNR & 3.51 & 6.25 & 8.13 & 9.61 & 13.52 & 18.78 \\
\hline & SDR & 3.14 & 5.97 & 8.09 & 9.24 & 13.18 & 17.16 \\
\hline & Seg SNR & 2.82 & 5.04 & 6.63 & 8.87 & 12.76 & 15.31 \\
\hline \multirow{3}{*}{$\mathrm{NMF}+\mathrm{OU}[12]$} & SNR & 3.11 & 3.68 & 7.25 & 8.65 & 12.74 & 17.24 \\
\hline & SDR & 2.72 & 3.12 & 7.02 & 8.27 & 11.86 & 14.13 \\
\hline & Seg SNR & 2.23 & 3.97 & 5.89 & 8.13 & 11.34 & 13.62 \\
\hline \multirow{3}{*}{ CUP-GG[9] } & SNR & 3.08 & 5.74 & 7.65 & 8.87 & 11.96 & 17.69 \\
\hline & SDR & 2.86 & 3.31 & 7.57 & 8.74 & 12.66 & 15.84 \\
\hline & Seg SNR & 2.64 & 4.86 & 6.75 & 8.52 & 12.21 & 14.76 \\
\hline \multirow{3}{*}{ CUP-NG[9] } & SNR & 2.98 & 4.34 & 7.64 & 9.27 & 12.98 & 17.42 \\
\hline & SDR & 2.78 & 3.38 & 7.17 & 8.68 & 12.58 & 15.21 \\
\hline & Seg SNR & 2.12 & 3.94 & 6.03 & 8.24 & 11.03 & 13.62 \\
\hline \multirow{3}{*}{ CUP[8] } & SNR & 3.17 & 3.47 & 7.48 & 8.97 & 12.65 & 16.93 \\
\hline & SDR & 2.54 & 3.16 & 7.09 & 8.57 & 12.38 & 15.24 \\
\hline & Seg SNR & 1.42 & 2.11 & 5.74 & 7.94 & 10.97 & 13.16 \\
\hline \multirow{3}{*}{ NR-NMF[14] } & SNR & 1.58 & 3.72 & 6.12 & 8.63 & 13.32 & 15.76 \\
\hline & SDR & 0.79 & 2.58 & 5.72 & 7.88 & 12.26 & 14.83 \\
\hline & Seg SNR & -2.37 & -0.64 & 2.86 & 6.12 & 10.62 & 13.67 \\
\hline \multirow{3}{*}{ WR-NMF[14] } & SNR & 1.19 & 3.64 & 5.85 & 8.54 & 12.67 & 15.58 \\
\hline & SDR & 0.23 & 2.28 & 5.75 & 7.76 & 11.58 & 15.02 \\
\hline & Seg SNR & -3.11 & -0.71 & 2.48 & 5.28 & 10.74 & 13.24 \\
\hline \multirow{3}{*}{ PR NMF [11] } & SNR & 0.14 & 2.18 & 3.97 & 7.57 & 11.24 & 15.31 \\
\hline & SDR & -2.01 & 1.28 & 3.09 & 6.79 & 10.07 & 12.95 \\
\hline & Seg SNR & -3.25 & -1.18 & 2.38 & 4.75 & 9.54 & 12.47 \\
\hline \multirow{3}{*}{ HRNR-SPU [13] } & SNR & -3.82 & -1.13 & 0.09 & 5.48 & 11.79 & 15.38 \\
\hline & SDR & -5.64 & -3.02 & -0.01 & 2.97 & 6.27 & 11.95 \\
\hline & Seg SNR & -7.89 & -3.85 & -0.82 & 2.98 & 7.05 & 10.15 \\
\hline \multirow{3}{*}{ HRNR[5] } & SNR & -4.82 & -1.64 & -0.09 & 5.68 & 10.69 & 13.54 \\
\hline & SDR & -6.98 & -2.47 & -0.14 & 2.89 & 9.59 & 11.38 \\
\hline & Seg SNR & -8.12 & -4.11 & -0.96 & 2.42 & 5.85 & 9.27 \\
\hline
\end{tabular}


Table 27. Comparison of Average PESQ, $\triangle$ PESQ performance measures for the 30 speech signals corrupted by street noise under Input SNRs of -10 $\mathrm{dB},-5 \mathrm{~dB}, 0 \mathrm{~dB}, 5 \mathrm{~dB}, 10 \mathrm{~dB}, 15 \mathrm{~dB}$

\begin{tabular}{|c|c|c|c|c|c|c|c|}
\hline $\begin{array}{l}\text { Input SNR in dB } \\
\text { Method }\end{array}$ & $\begin{array}{l}\text { Performance } \\
\text { Measure }\end{array}$ & -10 & -5 & $\mathbf{0}$ & 5 & 10 & 15 \\
\hline Without Processing & PESQ & 1.07 & 1.56 & 1.75 & 2.12 & 2.25 & 2.47 \\
\hline \multirow{2}{*}{$\begin{array}{c}\text { SE+CUP-GG+NR- } \\
\text { NMF+OU } \\
\end{array}$} & PESQ & 1.31 & 2.15 & 2.66 & 3.07 & 3.14 & 3.26 \\
\hline & $\triangle$ PESQ & 0.24 & 0.59 & 0.91 & 0.95 & 0.89 & 0.79 \\
\hline \multirow{2}{*}{$\begin{array}{c}\text { SE+CUP-GG+WR- } \\
\text { NMF+OU }\end{array}$} & PESQ & 1.31 & 2.13 & 2.62 & 3.02 & 3.12 & 3.29 \\
\hline & $\triangle$ PESQ & 0.24 & 0.57 & 0.87 & 0.9 & 0.87 & 0.82 \\
\hline \multirow{2}{*}{$\mathrm{SE}+\mathrm{CUP}-\mathrm{GG}+\boldsymbol{\beta}-\mathrm{NMF}+\mathrm{OU}$} & PESQ & 1.29 & 2.14 & 2.63 & 3.04 & 3.08 & 3.23 \\
\hline & $\triangle$ PESQ & 0.22 & 0.58 & 0.88 & 0.92 & 0.83 & 0.76 \\
\hline \multirow{2}{*}{ SE+CUP GG+NMF[9] } & PESQ & 1.3 & 2.01 & 2.61 & 2.94 & 3.04 & 3.18 \\
\hline & $\triangle$ PESQ & 0.23 & 0.45 & 0.86 & 0.82 & 0.79 & 0.71 \\
\hline \multirow{2}{*}{$\mathrm{NMF}+\mathrm{OU}[12]$} & PESQ & 1.16 & 1.91 & 2.43 & 2.82 & 2.86 & 3.07 \\
\hline & $\triangle$ PESQ & 0.09 & 0.35 & 0.68 & 0.7 & 0.61 & 0.6 \\
\hline \multirow{2}{*}{ CUP-GG[9] } & PESQ & 1.22 & 1.97 & 2.44 & 2.83 & 2.9 & 3.1 \\
\hline & $\triangle$ PESQ & 0.15 & 0.41 & 0.69 & 0.71 & 0.65 & 0.63 \\
\hline \multirow{2}{*}{ CUP-NG[9] } & PESQ & 1.19 & 1.94 & 2.41 & 2.8 & 2.87 & 3.07 \\
\hline & $\triangle$ PESQ & 0.12 & 0.38 & 0.66 & 0.68 & 0.62 & 0.6 \\
\hline \multirow{2}{*}{ CUP[8] } & PESQ & 1.15 & 1.9 & 2.38 & 2.73 & 2.83 & 2.99 \\
\hline & $\triangle$ PESQ & 0.08 & 0.34 & 0.63 & 0.61 & 0.58 & 0.52 \\
\hline \multirow{2}{*}{ WR NMF[14] } & PESQ & 1.2 & 1.95 & 2.36 & 2.79 & 2.86 & 3.03 \\
\hline & $\triangle$ PESQ & 0.13 & 0.39 & 0.61 & 0.67 & 0.61 & 0.56 \\
\hline \multirow{2}{*}{ NR NMF[14] } & PESQ & 1.18 & 1.93 & 2.39 & 2.81 & 2.87 & 3.06 \\
\hline & $\triangle$ PESQ & 0.11 & 0.37 & 0.64 & 0.69 & 0.62 & 0.59 \\
\hline \multirow{2}{*}{ PR NMF[11] } & PESQ & 1.14 & 1.85 & 2.22 & 2.6 & 2.7 & 2.89 \\
\hline & $\triangle$ PESQ & 0.07 & 0.29 & 0.47 & 0.48 & 0.45 & 0.42 \\
\hline \multirow{2}{*}{ HRNR-SPU[13] } & PESQ & 1.11 & 1.8 & 2.16 & 2.51 & 2.63 & 2.8 \\
\hline & $\triangle$ PESQ & 0.04 & 0.24 & 0.41 & 0.39 & 0.38 & 0.33 \\
\hline \multirow{2}{*}{ HRNR[5] } & PESQ & 1.09 & 1.74 & 2.11 & 2.41 & 2.51 & 2.7 \\
\hline & $\triangle$ PESQ & 0.02 & 0.18 & 0.36 & 0.29 & 0.26 & 0.23 \\
\hline
\end{tabular}

In table 27, the Comparison of Average PESQ, $\triangle \mathrm{PESQ}$ performance measures for the 30 speech signals corrupted by Street noise under input SNRs of $-10 \mathrm{~dB},-5 \mathrm{~dB}, 0 \mathrm{~dB}, 5$ $\mathrm{dB}, 10 \mathrm{~dB}, 15 \mathrm{~dB}$ is listed. It is observed that the, $\mathrm{SE}+$ CUP-GG + NR-NMF + OU provides improved performance than to all compared methods and it provides a $\triangle \mathrm{PESQ}$ value of 0.91 for the input signal corrupted under Street noise at OdB SNR. It is observed that SE+CUP-GG + NMF approach provides an PESQ improvement of 0.86. Also it is observed that CUP-GG estimators provides an improved $\triangle \mathrm{PESQ}$ value of 0.69 compared to posterior WR-NMF of 0.61 at $0 \mathrm{~dB}$ input SNR. It is noted that the Bayesian estimators shown comparable improvement under low SNRs than to traditional NMF approach. Also it is noted that combination of Bayesian estimator with NMF approach provides improved results in case of Street noise.

In table 28, the Comparison of Average STOI, \%STOI performance measures for the 30 speech signals corrupted by Street noise under Input SNRs of $-10 \mathrm{~dB},-5 \mathrm{~dB}, 0 \mathrm{~dB}, 5$ $\mathrm{dB}, 10 \mathrm{~dB}, 15 \mathrm{~dB}$ is listed. It is observed that \% STOI improvement of STOI values for SE + CUP - GG + NR $\mathrm{NMF}+\mathrm{OU}$ approach is $7.03,6.12,3.97,2.81,2.12,1.01$ and for CUP-GG estimator is 4.87, 3.63, 3.32, 1.64, 0.43, 0.21 and for WR-NMF approach is $2.83,2.24,1.97,0.78$, $0.43,0.24$ at input SNRs of $-10 \mathrm{~dB},-5 \mathrm{~dB}, 0 \mathrm{~dB}, 5 \mathrm{~dB}, 10$ $\mathrm{dB}, 15 \mathrm{~dB}$ respectively. It is noted that at $-10 \mathrm{~dB},-5 \mathrm{~dB}$ SNRs there is significant improvement in SE+CUPGG+NMF based approaches than to all other methods.

In table 29, the Comparison of Average SNR, SDR, Seg SNR performance measures for the 30 speech signals corrupted by Street noise under Input SNRs of $-10 \mathrm{~dB}$, $5 \mathrm{~dB}, 0 \mathrm{~dB}, 5 \mathrm{~dB}, 10 \mathrm{~dB}, 15 \mathrm{~dB}$ is listed. The proposed method SE+CUP-GG+NR-NMF+OU provides an SNR of $4.97,8.12,10.24,12.15,16.25,21.09$, and for SE+CUP-GG+NMF approach is 6.76, 5.34, 3.63, 2.45, $1.69,0.88$. The NR-NMF approach provides an SNR of $1.23,4.77,5.01,9.34,15.68,18.65$.

Table 28. Comparison of Average STOI, \% STOI performance measures for the 30 speech signals corrupted by street noise under Input SNRs of -10 $\mathrm{dB},-5 \mathrm{~dB}, 0 \mathrm{~dB}, 5 \mathrm{~dB}, 10 \mathrm{~dB}, 15 \mathrm{~dB}$

\begin{tabular}{|c|c|c|c|c|c|c|c|}
\hline $\begin{array}{l}\text { Input SNR in dB } \\
\text { Method }\end{array}$ & $\begin{array}{c}\text { Performance } \\
\text { Measure }\end{array}$ & -10 & -5 & $\mathbf{0}$ & 5 & 10 & 15 \\
\hline Without Processing & STOI & 0.3212 & 0.5672 & 0.6723 & 0.7562 & 0.8345 & 0.9321 \\
\hline \multirow{2}{*}{$\mathrm{SE}+\mathrm{CUP}-\mathrm{GG}+\mathrm{NR}-\mathrm{NMF}+\mathrm{OU}$} & $\%$ STOI & 7.03 & 6.12 & 3.97 & 2.81 & 2.12 & 1.01 \\
\hline & STOI & 0.3437 & 0.6019 & 0.6989 & 0.7774 & 0.8522 & 0.9415 \\
\hline \multirow{2}{*}{ SE+CUP-GG+WR-NMF+OU } & $\%$ STOI & 6.94 & 5.91 & 3.89 & 2.63 & 2.03 & 0.97 \\
\hline & STOI & 0.3435 & 0.6007 & 0.6984 & 0.7760 & 0.8514 & 0.9411 \\
\hline \multirow{2}{*}{$\mathrm{SE}+\mathrm{CUP}-\mathrm{GG}+\boldsymbol{\beta}-\mathrm{NMF}+\mathrm{OU}$} & $\%$ STOI & 6.89 & 5.72 & 3.76 & 2.51 & 1.92 & 0.91 \\
\hline & STOI & 0.3433 & 0.5996 & 0.6975 & 0.7752 & 0.8505 & 0.9405 \\
\hline
\end{tabular}




\begin{tabular}{|c|c|c|c|c|c|c|c|}
\hline \multirow{2}{*}{ SE+CUP GG+NMF[9] } & $\%$ STOI & 6.76 & 5.34 & 3.63 & 2.45 & 1.69 & 0.88 \\
\hline & STOI & 0.3429 & 0.5974 & 0.6967 & 0.7747 & 0.8486 & 0.9403 \\
\hline \multirow{2}{*}{$\mathrm{NMF}+\mathrm{OU}[12]$} & $\%$ STOI & 4.63 & 3.71 & 2.79 & 1.31 & 0.78 & 0.61 \\
\hline & STOI & 0.3360 & 0.5882 & 0.6910 & 0.7661 & 0.8410 & 0.9377 \\
\hline \multirow{2}{*}{ CUP-GG[9] } & $\%$ STOI & 4.87 & 3.63 & 3.32 & 1.64 & 0.43 & 0.21 \\
\hline & STOI & 0.3368 & 0.5877 & 0.69462 & 0.7686 & 0.8381 & 0.9340 \\
\hline \multirow{2}{*}{ CUP-NG[9] } & $\%$ STOI & 4.74 & 3.61 & 3.16 & 1.72 & 0.48 & 0.22 \\
\hline & STOI & 0.3364 & 0.5876 & 0.6935 & 0.7692 & 0.8385 & 0.9341 \\
\hline \multirow{2}{*}{ CUP[8] } & $\%$ STOI & 3.56 & 2.78 & 2.47 & 0.89 & 0.39 & 0.23 \\
\hline & STOI & 0.3326 & 0.5829 & 0.6889 & 0.7629 & 0.8377 & 0.9342 \\
\hline \multirow{2}{*}{ WR NMF[14] } & $\%$ STOI & 2.83 & 2.24 & 1.97 & 0.78 & 0.43 & 0.24 \\
\hline & STOI & 0.3302 & 0.5799 & 0.6855 & 0.7621 & 0.8380 & 0.9343 \\
\hline \multirow{2}{*}{ NR NMF[14] } & $\%$ STOI & 2.44 & 2.02 & 1.78 & 0.76 & 0.45 & 0.19 \\
\hline & STOI & 0.3289 & 0.5786 & 0.6842 & 0.7619 & 0.8382 & 0.9338 \\
\hline \multirow{2}{*}{ PR NMF[11] } & $\%$ STOI & 1.57 & 1.13 & 0.98 & 0.42 & 0.21 & 0.15 \\
\hline & STOI & 0.3262 & 0.5736 & 0.6788 & 0.7593 & 0.8362 & 0.9335 \\
\hline \multirow{2}{*}{ HRNR-SPU[13] } & $\%$ STOI & -2.32 & -2.01 & -1.12 & -1.56 & -1.09 & -1.1 \\
\hline & STOI & 0.3137 & 0.5557 & 0.6647 & 0.7443 & 0.8254 & 0.9218 \\
\hline \multirow{2}{*}{ HRNR[5] } & $\%$ STOI & -2.73 & -2.54 & -1.98 & -1.87 & -1.54 & -1.23 \\
\hline & STOI & 0.3124 & 0.5527 & 0.6589 & 0.7420 & 0.8216 & 0.9206 \\
\hline
\end{tabular}

Table 29. Comparison of Average SNR, SDR, Seg SNR performance measures for the 30 speech signals corrupted by street noise under Input SNRs of $-10 \mathrm{~dB},-5 \mathrm{~dB}, 0 \mathrm{~dB}, 5 \mathrm{~dB}, 10 \mathrm{~dB}, 15 \mathrm{~dB}$

\begin{tabular}{|c|c|c|c|c|c|c|c|}
\hline Method Input SNR in dB & $\begin{array}{l}\text { Performance } \\
\text { Measure }\end{array}$ & -10 & -5 & $\mathbf{0}$ & 5 & 10 & 15 \\
\hline \multirow{3}{*}{$\mathrm{SE}+\mathrm{CUP}-\mathrm{GG}+\mathrm{NR}-\mathrm{NMF}+\mathrm{OU}$} & SNR & 4.97 & 8.12 & 10.24 & 12.15 & 16.25 & 21.09 \\
\hline & SDR & 4.21 & 6.52 & 7.54 & 11.63 & 15.63 & 18.97 \\
\hline & Seg SNR & 3.83 & 6.34 & 7.58 & 10.98 & 14.79 & 17.13 \\
\hline \multirow{3}{*}{$\mathrm{SE}+\mathrm{CUP}-\mathrm{GG}+\mathrm{WR}-\mathrm{NMF}+\mathrm{OU}$} & SNR & 4.21 & 7.58 & 10.31 & 11.61 & 16.57 & 20.85 \\
\hline & SDR & 3.92 & 6.52 & 9.12 & 11.23 & 14.95 & 19.25 \\
\hline & Seg SNR & 3.64 & 5.57 & 7.11 & 9.58 & 13.32 & 15.36 \\
\hline \multirow{3}{*}{$\mathrm{SE}+\mathrm{CUP}-\mathrm{GG}+\boldsymbol{\beta}-\mathrm{NMF}+\mathrm{OU}$} & SNR & 4.59 & 7.08 & 10.21 & 11.64 & 15.96 & 20.48 \\
\hline & SDR & 3.85 & 6.24 & 8.98 & 10.52 & 14.56 & 16.88 \\
\hline & Seg SNR & 3.56 & 5.41 & 6.98 & 9.45 & 13.11 & 15.28 \\
\hline \multirow{3}{*}{ SE+CUP GG+NMF[9] } & SNR & 4.53 & 7.32 & 9.85 & 11.56 & 14.89 & 19.95 \\
\hline & SDR & 3.74 & 5.98 & 8.68 & 10.35 & 14.38 & 16.78 \\
\hline & Seg SNR & 3.32 & 5.57 & 7.31 & 9.64 & 13.28 & 15.48 \\
\hline \multirow{3}{*}{$\mathrm{NMF}+\mathrm{OU}[12]$} & SNR & 2.75 & 3.89 & 7.84 & 11.14 & 14.84 & 18.58 \\
\hline & SDR & 2.15 & 3.07 & 6.87 & 9.12 & 13.24 & 15.28 \\
\hline & Seg SNR & 1.42 & 1.78 & 6.75 & 8.56 & 12.2 & 14.58 \\
\hline \multirow{3}{*}{ CUP-GG[9] } & SNR & 4.11 & 6.76 & 8.85 & 10.76 & 15.54 & 19.25 \\
\hline & SDR & 3.78 & 6.77 & 8.59 & 10.64 & 13.87 & 16.27 \\
\hline & Seg SNR & 3.27 & 5.34 & 7.07 & 8.86 & 12.54 & 15.04 \\
\hline \multirow{3}{*}{ CUP-NG[9] } & SNR & 4.14 & 5.67 & 8.46 & 10.29 & 13.95 & 19.12 \\
\hline & SDR & 2.95 & 3.84 & 7.65 & 8.97 & 12.97 & 18.15 \\
\hline & Seg SNR & 2.08 & 4.37 & 6.62 & 8.49 & 11.98 & 13.98 \\
\hline \multirow{3}{*}{ CUP $[8]$} & SNR & 2.26 & 3.57 & 7.86 & 9.42 & 13.57 & 18.12 \\
\hline & SDR & 2.58 & 2.96 & 6.57 & 8.54 & 12.46 & 14.68 \\
\hline & Seg SNR & 1.59 & 2.07 & 5.84 & 7.42 & 10.76 & 13.74 \\
\hline \multirow{3}{*}{ NR-NMF[14] } & SNR & 1.23 & 4.77 & 5.01 & 9.34 & 15.68 & 18.65 \\
\hline & SDR & 0.92 & 2.78 & 4.67 & 10.45 & 14.38 & 18.24 \\
\hline & Seg SNR & -3.12 & -0.87 & 3.21 & 6.64 & 10.75 & 13.54 \\
\hline \multirow{3}{*}{ WR-NMF[14] } & SNR & 0.58 & 2.98 & 4.37 & 9.32 & 14.86 & 18.24 \\
\hline & SDR & 0.15 & 1.97 & 3.97 & 9.14 & 13.75 & 1.7 .67 \\
\hline & Seg SNR & -3.65 & -1.38 & 2.41 & 4.85 & 10.56 & 13.08 \\
\hline \multirow{3}{*}{ PR NMF [11] } & SNR & 0.09 & 1.78 & 4.06 & 8.21 & 12.69 & 17.12 \\
\hline & SDR & -1.89 & 1.23 & 2.97 & 6.86 & 12.07 & 15.14 \\
\hline & Seg SNR & -4.14 & -2.42 & 1.28 & 3.76 & 8.79 & 11.84 \\
\hline \multirow{3}{*}{ HRNR-SPU [13] } & SNR & 1.87 & 1.74 & 3.87 & 6.76 & 12.25 & 16.86 \\
\hline & SDR & 1.12 & 1.51 & 2.45 & 5.31 & 10.45 & 14.12 \\
\hline & Seg SNR & 0.24 & 1.14 & 1.98 & 2.54 & 6.31 & 8.68 \\
\hline \multirow{3}{*}{ HRNR[5] } & SNR & 1.14 & 1.45 & 2.12 & 6.25 & 10.65 & 15.89 \\
\hline & SDR & 0.78 & 1.25 & 2.14 & 5.31 & 10.37 & 15.11 \\
\hline & Seg SNR & 0.09 & 0.67 & 1.54 & 2.11 & 5.75 & 8.96 \\
\hline
\end{tabular}




\section{CONCLUSION}

Statistical approaches and NMF approaches shows significant performance for speech Enhancement task. The assumption of speech spectral coefficients and noise spectral coefficients as super Gaussian provides improved PESQ, STOI, SNR values than to traditional speech enhancement methods. Template based Non-Negative Matrix Factorization (NMF) approach provides better results than to traditional speech enhancement methods for Non-Stationary signals like speech. By combining the advantages of both statistical approaches and NMF approach the performance measures PESQ, SNR and STOI are improved significantly. This work gives the importance of statistical approaches, NMF approach and proposed work by combining statistical approaches and NMF. It is observed that combination of statistical and variants of NMF provides better noise enhanced speech.

\section{APPENDIX}

\section{APPENDIX A}

\section{(Derivation of CUP Estimator by assuming Nagakami PDF for speech prior and Generalized Gamma Prior for} noise )

The CUP estimator by assuming speech as Nagakami prior and noise as GGD is derived as in [8]

Assume the speech prior as Nagakami PDF as

$$
p_{A}(a)=\frac{2}{\Gamma(k)}\left(\frac{k}{\sigma_{S}^{2}}\right)^{k} a^{2 k-1} \exp \left(-\frac{k}{\sigma_{S}^{2}} a^{2}\right)
$$

Assume the noise prior as GGD as

$$
p_{Y \mid S}(y \mid s)=\frac{2 \mu^{v}}{\Gamma(v) \sigma_{N}^{2}}\left(\frac{a}{\sigma_{N}^{2}}\right)^{2 v-1} \exp \left(-\mu\left(\frac{y-a}{\sigma_{N}^{2}}\right)^{2}\right)
$$

The CUP estimator is derived as in [8] using

$$
\begin{aligned}
& \hat{S}^{(\beta)}= E\left(A^{\beta} e^{j \Phi_{S}} \mid y, \tilde{\phi}_{S}\right)= \\
&=\frac{a^{\beta} e^{j \phi_{S}} \int_{0}^{2 \pi} \int_{0}^{\infty} \frac{2}{\Gamma(k)}\left(\frac{k}{\sigma_{S}^{2}}\right)^{k} a^{2 k-1} e \operatorname{xp}\left(-\frac{k}{\sigma_{S}^{2}} a^{2}\right) \frac{2 \mu^{v}}{\Gamma(v) \sigma_{N}^{2}}\left(\frac{a}{\sigma_{N}^{2}}\right)^{2 v-1} \frac{2 \mu^{v}}{\Gamma(v) \sigma_{N}^{2}}\left(\frac{a}{\sigma_{N}^{2}}\right)^{2 v-1}}{\int_{0}^{2 \pi} \int_{0}^{\infty} \frac{2}{\Gamma(k)}\left(\frac{k}{\sigma_{S}^{2}}\right)^{k} a^{2 k-1} \exp \left(-\frac{k}{\sigma_{S}^{2}} a^{2}\right) \frac{2 \mu^{v}}{\Gamma(v) \sigma_{N}^{2}}\left(\frac{a}{\sigma_{N}^{2}}\right)^{2 v-1} \frac{2 \mu^{v}}{\Gamma(v) \sigma_{N}^{2}}\left(\frac{a}{\sigma_{N}^{2}}\right)^{2 v-1}} \\
& \times \frac{\exp \left(-\mu\left(\frac{y-a}{\sigma_{N}^{2}}\right)^{2}\right) p_{\Phi_{S} \mid \tilde{\phi}_{S}}\left(\phi_{S} \mid \tilde{\phi}_{S}\right) d a d \phi_{S}}{\exp \left(-\mu\left(\frac{y-a}{\sigma_{N}^{2}}\right)^{2}\right) p_{\Phi_{S} \mid \tilde{\phi}_{S}}\left(\phi_{S} \mid \tilde{\phi}_{S}\right) d a d \phi_{S}}
\end{aligned}
$$




$$
\begin{aligned}
& \hat{S}^{(\beta)}=E\left(A^{\beta} e^{j \Phi_{S}} \mid y, \tilde{\phi}_{S}\right)= \\
& =\frac{e^{j \phi_{S}} \int_{0}^{2 \pi} \frac{4 \mu^{v} k^{k}}{\Gamma(k) \Gamma(v)\left(\sigma_{S}^{2}\right)^{k} \sigma_{N}^{2}\left(\sigma_{N}^{2}\right)^{2 v-1}} \exp \left(\frac{r^{2}}{\sigma_{N}^{2}}\right)}{4 \mu^{v} k^{k}} \\
& \int_{0}^{2 \pi} \frac{r^{2}}{\Gamma(k) \Gamma(v)\left(\sigma_{S}^{2}\right)^{k} \sigma_{N}^{2}\left(\sigma_{N}^{2}\right)^{2 v-1}} \exp \left(\frac{\sigma_{N}^{2}}{\sigma_{N}^{2}}\right) \\
& \quad \times \frac{\int_{0}^{\infty} a^{2 k+2 v+\beta-2} \exp \left(-\left(\frac{k}{\sigma_{S}^{2}}+\frac{\mu}{\sigma_{N}^{2}}\right) a^{2}-\left(\frac{2 \mu r \cos \Delta \phi}{\sigma_{N}^{2}}\right) a\right) p_{\Phi_{S} \mid \tilde{\phi}_{S}}\left(\phi_{S} \mid \tilde{\phi}_{S}\right) d a d \phi_{S}}{\int_{0}^{\infty} a^{2 k+2 v-2} \exp \left(-\left(\frac{k}{\sigma_{S}^{2}}+\frac{\mu}{\sigma_{N}^{2}}\right) a^{2}-\left(\frac{2 \mu r \cos \Delta \phi}{\sigma_{N}^{2}}\right) a\right) p_{\Phi_{S} \mid \tilde{\phi}_{S}}\left(\phi_{S} \mid \tilde{\phi}_{S}\right) d a d \phi_{S}}
\end{aligned}
$$

After rearranging and simplification using Gradshteyn and Ryzhik (2007 Eq. (3.462.1))

$$
\int_{0}^{\infty} x^{p-1} e^{-\lambda x^{2}-\gamma x}=(2 \lambda)^{\frac{-p}{2}} \Gamma(p) \exp \left(\frac{\gamma^{2}}{8 \lambda}\right) D_{-p}\left(\frac{\gamma}{2 \sqrt{\lambda}}\right)
$$

By Comparing, the obtained terms are

$$
p=2 k+2 v-1 ; \lambda=\frac{k \sigma_{N}^{2}+\mu \sigma_{S}^{2}}{\sigma_{S}^{2} \sigma_{N}^{2}} \gamma=\frac{2 r \mu \cos \Delta \phi}{\sigma_{N}^{2}}
$$

By Substituting all parameters after simplification

$$
\begin{gathered}
\hat{S}^{(\beta)}=E\left(A^{\beta} e^{j \Phi_{S}} \mid y, \tilde{\phi}_{S}\right)= \\
=\frac{\int_{0}^{2 \pi} \frac{4 \mu^{v} k^{k} \Gamma(2 k+2 v+\beta-1)}{\Gamma(k) \Gamma(v)\left(\sigma_{S}^{2}\right)^{k}\left(\sigma_{N}^{2}\right)^{2 v}}\left(2\left(\frac{k}{\xi \sigma_{N}^{2}}+\frac{\xi \mu}{\sigma_{S}^{2}}\right)\right)^{-\left(k+v+\frac{\beta+1}{2}\right)} e^{j \phi_{S}} \exp \left(\frac{r^{2}}{\sigma_{N}^{2}}\right)}{\int_{0}^{2 \pi} \frac{4 \mu^{v} k^{k} \Gamma(2 k+2 v-1)}{\Gamma(k) \Gamma(v)\left(\sigma_{S}^{2}\right)^{k}\left(\sigma_{N}^{2}\right)^{2 v}}\left(2\left(\frac{k}{\xi \sigma_{N}^{2}}+\frac{\xi \mu}{\sigma_{S}^{2}}\right)\right)^{-\left(k+v+\frac{1}{2}\right)} \exp \left(\frac{r^{2}}{\sigma_{N}^{2}}\right)} \\
\quad \times \frac{\exp \left(\frac{v^{2}}{2}\right) D_{-(2 v+2 k+\beta-1)}(v) p_{\Phi_{S} \mid \tilde{\phi}_{S}}\left(\phi_{S} \mid \tilde{\phi}_{S}\right) d \phi_{S}}{\hat{S}^{(\beta)}=E\left(A^{\beta} e^{j \Phi_{S}} \mid y, \tilde{\phi}_{S}\right)=} \\
\left.=\frac{\Gamma(2 k+2 v+\beta-1)\left(\frac{v^{2}}{2}\right) D_{-(2 v+2 k-1)}(v) p_{\Phi_{S} \mid \tilde{\phi}_{S}}\left(\phi_{S} \mid \tilde{\phi}_{S}\right) d \phi_{S}}{\Gamma(2 k+2 v-1)}\left(\frac{k}{\xi \sigma_{N}^{2}}+\frac{\xi \mu}{\sigma_{S}^{2}}\right)\right)^{-\left(\frac{\beta}{2}\right)} \frac{\int_{0}^{2 \pi} e^{j \phi_{S}} \exp \left(\frac{r^{2}}{\sigma_{N}^{2}}\right) \exp \left(\frac{v^{2}}{2}\right) D_{-(2 v+2 k+\beta-1)}(v) p_{\Phi_{S} \mid \tilde{\phi}_{S}}\left(\phi_{S} \mid \tilde{\phi}_{S}\right) d \phi_{S}}{\int_{0}^{2 \pi} \exp \left(\frac{r^{2}}{\sigma_{N}^{2}}\right) \exp \left(\frac{v^{2}}{2}\right) D_{-(2 v+2 k-1)}(v) p_{\Phi_{S} \mid \tilde{\phi}_{S}}\left(\phi_{S} \mid \tilde{\phi}_{S}\right) d \phi_{S}}
\end{gathered}
$$




\section{APPENDIX B}

(Derivation of CUP Estimator by assuming Gamma PDF for speech prior and Generalized Gamma Prior for noise priori)

The CUP estimator by assuming speech as Gamma prior and noise as GGD is derived as in [8]

Assume the speech prior as Gamma PDF as

$$
p_{A}(a)=\frac{1}{\theta^{k} \Gamma(k)} a^{k-1} \exp \left(-\frac{a}{\theta}\right)
$$

Assume the noise prior as GGD as

$$
p_{Y \mid S}(y \mid s)=\frac{2 \mu^{v}}{\Gamma(v) \sigma_{N}^{2}}\left(\frac{a}{\sigma_{N}^{2}}\right)^{2 v-1} \exp \left(-\mu\left(\frac{y-a}{\sigma_{N}^{2}}\right)^{2}\right)
$$

The CUP estimator is derived as in [20] and from Gradshteyn and Ryzhik (2007 Eq. (3.462.1))

$$
\int_{0}^{\infty} x^{p-1} e^{-\lambda x^{2}-\gamma x}=(2 \lambda)^{\frac{-p}{2}} \Gamma(p) \exp \left(\frac{\gamma^{2}}{8 \lambda}\right) D_{-p}\left(\frac{\gamma}{2 \sqrt{\lambda}}\right)
$$

By comparing the parameters we obtain parameters

$$
p=2 v+k+\beta-1 ; \lambda=\frac{\mu}{\sigma_{N}^{2}} ; \gamma=\frac{1}{\sigma_{S}}-\frac{2 \mu y \cos \left(\phi_{S}-\phi_{Y}\right)}{\sigma_{N}^{2}}
$$

The Numerator term is obtained finally as

$$
\begin{aligned}
\int_{0}^{2 \pi} \frac{2 \mu^{v}}{\Gamma(k) \theta^{k} \Gamma(v) \sigma_{N}^{2}}\left(\frac{1}{\sigma_{N}^{2}}\right)^{2 v-1}\left(\frac{2 \mu}{\sigma_{N}^{2}}\right)^{-\left(\frac{2 v+k+\beta-1}{2}\right)} \Gamma(2 v+k+\beta-1) \exp \left(\frac{\left(\frac{1}{\sigma_{S}}-\frac{2 \mu y \cos \left(\phi_{S}-\phi_{Y}\right)}{\sigma_{N}^{2}}\right)^{2}}{8 \mu / \sigma_{N}^{2}}\right) \\
\\
\times D_{-(2 v+k+\beta-1)}\left(\frac{\frac{1}{\sigma_{S}}-\frac{2 \mu y \cos \left(\phi_{S}-\phi_{Y}\right)}{\sigma_{N}^{2}}}{2 \sqrt{\mu / \sigma_{N}^{2}}}\right)
\end{aligned}
$$

Let $v=\left(\frac{-2 y \mu \sigma_{S} \cos \left(\phi_{S}-\phi_{Y}\right)+\sigma_{N}^{2}}{2 \sigma_{N}^{2} \sigma_{S} \sqrt{\mu}}\right) \sigma_{N}$

Divide Numerator for above $v$ with $\sigma_{S}$ and $\sigma_{N}^{2}$ then $v=\frac{\sigma_{S}-2 y \mu \xi \cos \left(\phi_{S}-\phi_{Y}\right)}{2 \xi \sigma_{N} \sqrt{\mu}}$

Finally the estimated signal using (3) and using simplified equation as

$$
\frac{2 \mu^{v}}{\Gamma(k) \theta^{k} \Gamma(v)\left(\sigma_{N}^{2}\right)^{2 v}}\left(\frac{2 \mu}{\sigma_{N}^{2}}\right)^{-\left(v+\frac{k+\beta-1}{2}\right)} \Gamma(2 v+k+\beta-1) \exp \left(\frac{v^{2}}{2}\right) D_{-(2 v+k+\beta-1)}(v)
$$




$$
\begin{aligned}
& \hat{S}^{(\beta)}=E\left(A^{\beta} e^{j \Phi_{S}} \mid y, \tilde{\phi}_{S}\right)= \\
& =\frac{\int_{0}^{2 \pi} \frac{2 \mu^{v}}{\Gamma(k) \theta^{k} \Gamma(v) \sigma_{N}^{2}}\left(\frac{1}{\sigma_{N}^{2}}\right)^{2 v-1}\left(\frac{2 \mu}{\sigma_{N}^{2}}\right)^{-\left(\frac{2 v+k+\beta-1}{2}\right)} \Gamma(2 v+k+\beta-1) \exp \left(\frac{\left(\frac{1}{\sigma_{S}}-\frac{2 \mu y \cos \left(\phi_{S}-\phi_{Y}\right)}{\sigma_{N}^{2}}\right)^{2}}{8 \mu / \sigma_{N}^{2}}\right)}{\int_{0}^{2 \pi} \frac{2 \mu^{v}}{\Gamma(k) \theta^{k} \Gamma(v) \sigma_{N}^{2}}\left(\frac{1}{\sigma_{N}^{2}}\right)^{2 v-1}\left(\frac{2 \mu}{\sigma_{N}^{2}}\right)^{-\left(\frac{2 v+k-1}{2}\right)} \Gamma(2 v+k-1) \exp \left(\frac{\left(\frac{1}{\sigma_{S}}-\frac{2 \mu y \cos \left(\phi_{S}-\phi_{Y}\right)}{\sigma_{N}^{2}}\right)^{2}}{8 \mu / \sigma_{N}^{2}}\right)} \\
& \times \frac{D_{-(2 v+k+\beta-1)}\left(\frac{\frac{1}{\sigma_{S}}-\frac{2 \mu y \cos \left(\phi_{S}-\phi_{Y}\right)}{\sigma_{N}^{2}}}{2 \sqrt{\mu / \sigma_{N}^{2}}}\right) p_{\Phi_{S} \mid \tilde{\phi}_{S}}\left(\phi_{S} \mid \tilde{\phi}_{S}\right) d \phi_{S}}{D_{-(2 v+k-1)}\left(\frac{\frac{1}{\sigma_{S}}-\frac{2 \mu y \cos \left(\phi_{S}-\phi_{Y}\right)}{\sigma_{N}^{2}}}{2 \sqrt{\mu / \sigma_{N}^{2}}}\right) p_{\Phi_{S} \mid \tilde{\phi}_{S}}\left(\phi_{S} \mid \tilde{\phi}_{S}\right) d \phi_{S}} \\
& \hat{S}^{(\beta)}=E\left(A^{\beta} e^{j \Phi_{S}} \mid y, \tilde{\phi}_{S}\right)= \\
& =\frac{\int_{0}^{2 \pi}\left(\frac{2 \mu}{\sigma_{N}^{2}}\right)^{-\left(v+\frac{k+\beta-1}{2}\right)} \Gamma(2 v+k+\beta-1) \exp \left(\frac{v^{2}}{2}\right) D_{-(2 v+k \beta-1)}(v) p_{\Phi_{S} \mid \tilde{\phi}_{S}}\left(\phi_{S} \mid \tilde{\phi}_{S}\right) d \phi_{S}}{\int_{0}^{2 \pi}\left(\frac{2 \mu}{\sigma_{N}^{2}}\right)^{-\left(v+\frac{k-1}{2}\right)} \Gamma(2 v+k-1) \exp \left(\frac{v^{2}}{2}\right) D_{-(2 v+k-1)}(v) p_{\Phi_{S} \mid \tilde{\phi}_{S}}\left(\phi_{S} \mid \tilde{\phi}_{S}\right) d \phi_{S}} \\
& \hat{S}^{(\beta)}=E\left(A^{\beta} e^{j \Phi_{S}} \mid y, \tilde{\phi}_{S}\right)= \\
& =\frac{\Gamma(2 v+k+\beta-1)}{\Gamma(2 v+k-1)}\left(\frac{2 \mu}{\sigma_{N}^{2}}\right)^{-\left(\frac{\beta}{2}\right)} \frac{\int_{0}^{2 \pi} e^{j \phi_{S}} \exp \left(\frac{v^{2}}{2}\right) D_{-(2 v+k \beta-1)}(v) p_{\Phi_{S} \mid \tilde{\phi}_{S}}\left(\phi_{S} \mid \tilde{\phi}_{S}\right) d \phi_{S}}{\int_{0}^{2 \pi} \exp \left(\frac{v^{2}}{2}\right) D_{-(2 v+k-1)}(v) p_{\Phi_{S} \mid \tilde{\phi}_{S}}\left(\phi_{S} \mid \tilde{\phi}_{S}\right) d \phi_{S}}
\end{aligned}
$$

\section{REFERENCES}

[1] R. Martin, "Speech enhancement based on minimum mean-square error estimation and super-Gaussian priors," IEEE Trans. Speech Audio Process., vol. 13, no. 5, pp. 845-856, Sep. 2005.

[2] C.H.You, S. N.Koh, and S. Rahardja, " $\beta$-order MMSE spectral amplitude estimation for speech enhancement," IEEE Trans. Speech Audio Process., vol. 13, no. 4, pp. 475-486, Jul. 2005

[3] B. Fodor, T. Fingscheidt, "MMSE speech enhancement under speech presence uncertainty assuming (generalized) gamma speech prioris throughout" IEEE International conf. on Acoustics, Speech and Signal Processing (ICASSP), pp.4033-4036, 25-30 March, 2012

[4] Lotter, T., Vary, P., Jan. 2005. Speech enhancement by MAP spectral amplitude estimation using a super-
Gaussian speech model. EURASIP J. Appl. Signal Process. 2005 (7), 1110-1126

[5] Cyril Plapous, Clande Marro, Pascal Scalart, "Speech enhancement using Harmonic Regeneration" IEEE conf. on Acoustics, speech, and signal processing (ICASSP),Vol.1, pp. 157-160, 18-23 March, 2005

[6] K. Paliwal, K. W'ojcicki, and B. Shannon, "The importance of phase in speech enhancement," Speech Commun., vol. 53, no. 4, pp. 465-494, Apr.2011

[7] Gerkmann, T., Krawczyk, M., Feb. 2013. MMSE-optimal spectral amplitude estimation given the STFT-phase. IEEE Signal Process. Lett. 20 (2), 129- 132.

[8] T. Gerkman, "Bayesian estimation of clean speech spectral coefficients given a priori knowledge of the phase," IEEE Trans, Signal Process., vol. 62, no. 16, pp. 4199-4208, Aug. 2014

[9] Ravi Kumar. K, P. V. Subbaiah, “ Speech enhancement using MMSE estimation of amplitude and complex speech 
spectral coefficients under phase uncertainty" in Speech Communication journal, Vol.96, pp.10-27, 2018.

[10] D. D. Lee, H. S. Seung, "Algorithms for non-negative matrix factorization" in Proc. Advances in Neural information processing systems (NIPS)., pp. 556 - 562, 2001.

[11] N. J. Bryan, G. J. Mysore, “ An efficient posterior regularized latent variable model for interactive sound source separation, " in International Conference of Machine Learning (ICML)., pp.208 - 216, June 2013

[12] Kwon Kisoo , Jong Won Shin , Nam Soo Kim "NMFbased speech enhancement using bases update". Sig Process Lett IEEE 2015;22(4):450-4

[13] Ravi Kumar K, P.V. Subbaiah, "Enhancement of noisy speech using sub-band harmonic regeneration and speech presence uncertain estimator" in Proc. IEEE International conference on Recent Trends in Electronics, Information \& Communication Technology January, 2017

[14] Ravi Kumar, P.V. Subbaiah " Posteriori Regularization based Non-Negative Matrix Factorization approach for

[15] Speech Enhancement, in International Journal of Innovative Technology and Exploring Engineering, V01.8, Issue-5S, March, 2019

\section{Authors' Profiles}

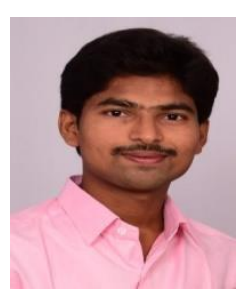

Ravi Kumar Kandagatla was born in Markapur, India in 1988. He received the Bachelor of Technology degree from Jawaharlal Nehru Technological University, Kakinada in 2009 and received Master of Technology in Digital Electronics and Communication Systems from Jawaharlal Nehru Technological University, Kakinada in 2011. He is Currently working as a Research Scholar at JNTUK, Kakinada and also working as Assistant professor in Lakireddy Balireddy College of Engineering, Mylavaram, India.
He has 7 years of teaching experience. He has 4 International publications. His interest area of research is speech processing .

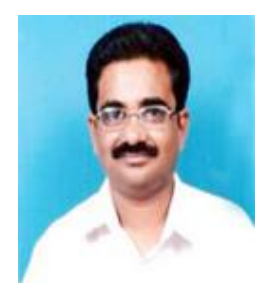

Dr. P. V Subbaiah was graduated in ECE from Bangalore University and received his Master's Degree from Andhra University, Visakhapatnam in 1982. JNTU, Hyderabad has conferred Ph.D degree on P.V. Subbaiah for his work on Microwave Antenna Test Facilities in the year 1996. $\mathrm{He}$ has vast teaching experience of 36 years in different reputed Institutions as Assistant Professor, Associate Professor, Professor and Head of the Department and Principal. Presently, he is the Professor of ECE at V.R. Siddhartha Engineering College, Vijayawada and discharging his duty as the Controller of Examinations. He was formerly the Coordinator of World Bank funded TEQIP Project since 2014. His areas of interest include Microwave Antennas, Smart Antennas and Communications. He has published more than 100 research papers in National and International Journals and Conferences of repute. Ten research scholars have received their $\mathrm{Ph} . \mathrm{D}$ degree under his supervision and presently guiding two more scholars for their $\mathrm{Ph} \mathrm{D}$. He is the Member and Fellow of various professional societies namely ISTE, BMESI, IETE and IE (I). He was the recipient of Sir Thomas ward Gold Prize from The Institution of Engineers (India).

How to cite this paper: Ravi Kumar Kandagatla, P V Subbaiah, "Performance Analysis of Statistical Approaches and NMF Approaches for Speech Enhancement", International Journal of Image, Graphics and Signal Processing(IJIGSP), Vol.11, No.7, pp. 9-38, 2019.DOI: 10.5815/ijigsp.2019.07.02 